THE DIFFERENTIAL CROSS SECTION DISTRIBUTIONS OF $D^{* \pm}$ AND $D^{0} / \overline{D^{0}}$ IN $\pi^{ \pm}$- NUCLEON INTERACTIONS

BY

\title{
ALI RAFATIAN
}

B.A., University of Tennessee, 1980

\author{
A Dissertation \\ Submitted to the Faculty of \\ The University of Mississippi \\ in Partial Fulfillment of the Requirements \\ for the Degree of Doctor of Philosophy \\ in the Department of Physics
}

The University of Mississippi

December 1993 


\author{
ABSTRACT \\ THE DIFFERENTIAL CROSS SECTION DISTRIBUTIONS OF $D^{* \pm}$ \\ AND $D^{0} / \overline{D^{0}}$ IN $\pi^{ \pm}$- NUCLEON INTERACTIONS \\ RAFATIAN, ALI. B.A., University of Tennessee, 1980. \\ Ph.D., University of Mississippi, 1993. \\ Dissertation directed by Dr. James J. Reidy.
}

Fermilab E769, a high statistics charm hadroproduction experiment, collected approximately $370 \times 10^{6}$ events during the fixed target run period of 1987-88. Using the Tagged Photon Spectrometer, the experiment was performed with a $250 \mathrm{GeV}$ beam of mixed hadrons $\left(\pi^{ \pm}, K^{ \pm}, p\right)$ interacting with a segmented foil target of Be, $\mathrm{Al}, \mathrm{Cu}$, and $\mathrm{W}$. The use of different beam particle types and polarities allowed E769 to explore the flavor dependence of charm hadroproduction while the choice of different target materials afforded the experiment a measurement of the atomic mass dependence. Using samples of $388 \pm 31 D^{0} / \overline{D^{0}}$ and $127 \pm 14 D^{* \pm}\left(\pi^{ \pm} D^{0} / \overline{D^{0}}\right)$ events produced from $\pi^{ \pm}$beam interactions, the shape parameters of the differential cross section distributions in fractional longitudinal momentum $\left(x_{F}\right)$ and in transverse momentum $\left(P_{t}\right)$ were determined. The final state of $D^{0}$ in both samples is the hadronic 4-prong $\left(K^{-} \pi^{+} \pi^{-} \pi^{+}\right)$decay mode. Fitting $d \sigma / d x_{F}$ distributions to the functional form $\left(1-x_{F}\right)^{n}$ yields values of $n=4.2 \pm 0.5$ for $D^{0} / \overline{D^{0}}$, and $n=2.5 \pm 0.7$ for $D^{* \pm}$. For $d \sigma / d P_{t}^{2}$ distributions, the fit to the functional form $\exp \left(-b P_{t}^{2}\right)$ gives values of $b=1.00 \pm 0.09(\mathrm{GeV} / \mathrm{c})^{-2}$ for $D^{0} / \overline{D^{0}}$, and $b=0.62 \pm 0.10(\mathrm{GeV} / \mathrm{c})^{-2}$ for $D^{* \pm}$. The difference in $n$ values for the non-leading versus the leading samples of $D^{*}$ production are measured to be $1.7 \pm 1.4$, indicative of a weak leading particle effect. Finally, the asymmetry for leading versus non-leading $D^{*}$ 's was determined to be $0.20 \pm 0.14$. 


\section{Acknowledgements}

There are a number of people who have directly or indirectly contributed to this work. The credits go to:

- all the members of E769 collaboration at Fermilab who made this experiment a success;

- the members of my thesis committee: Drs. Reidy, Cremaldi, Summers, Spalding, and Miller, for their advice and helpful comments;

- my colleagues in the High Energy Physics Group at the University of Mississippi, for their encouragement and assistance.

Special thanks go to:

- Jeff Spalding for his wonderful supervision of this work, his constant support, and his friendship;

- Steve Bracker who started me on PCOS III, and taught me how to use CAMAC;

- Robert Jedicke, Colin Gay, and Zhongxin Wu, whose dissertations were great sources of information;

- Lisa chen-Tokarek, for both her professional suppport and personal friendship;

- Krish Gounder, my colleague and roommate, for his support and delicious spicy cooking;

- Danying Yi, my colleague and very dear friend, for being always willing to lend an ear;

- and Dianne Jackson, for her love and support, and for her patience in the last few months.

Finally, I would like to dedicate this work to the most loving parents, Mehdi and Tala, who have nourished me with their love and their lives. 


\section{Contents}

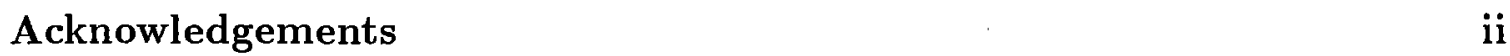

List of Tables vi vi vis

List of Figures viii

1 Introduction 1

1.1 Early Experiments ................... 1

1.2 Fermilab E769 .................... 4

2 Theory 5

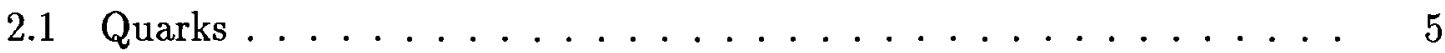

2.2 Quantum Chromodynamics (QCD) .............. 6

2.3 Charm Cross Section .................. 8

2.3.1 Structure Functions ............... 9

2.3.2 Parton-Parton Cross Section . . . . . . . . . . . 10

2.3.3 Fragmentation Functions .............. 14

2.3.4 $x_{F}$ Distribution of Charm Hadron Cross Section . . . . . . . 17

3 Experiment $\quad 19$

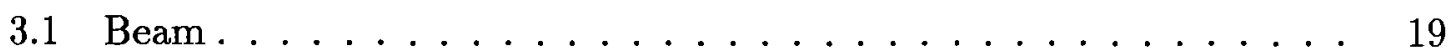

3.1.1 Primary Proton Beam ............... 19

3.1.2 Secondary Hadron Beam . . . . . . . . . . . . 20

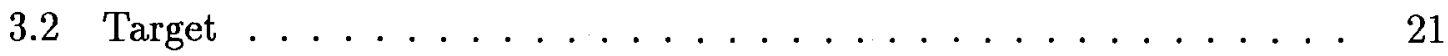


3.3 E769 Detector System . . . . . . . . . . . . . . . . . . 24

3.3.1 Čerenkov Counters . . . . . . . . . . . . . . . . 24

3.3.2 Transition Radiation Detector (TRD) . . . . . . . . . . 29

3.3.3 Multiwire Proportional Chambers (MWPC) . . . . . . . 32

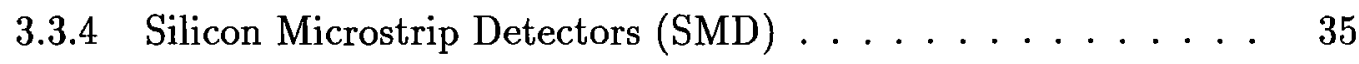

3.3.5 Drift Chamber $(\mathrm{DC}) \ldots \ldots \ldots \ldots \ldots$

3.3.6 Analysis Magnets . . . . . . . . . . . . . . . . . 40

3.3.7 Electromagnetic (EM) Calorimeter . . . . . . . . . 40

3.3.8 Hadronic Calorimeter . . . . . . . . . . . . . . . . . 41

3.4 Trigger . . . . . . . . . . . . . . . 43

3.5 Data Acquisition System . . . . . . . . . . . . . . 44

3.5.1 Hardware Configuration . . . . . . . . . . . . 47

3.5 .2 On-Line Monitoring . . . . . . . . . . . . . 48

4 Analysis $\quad 49$

4.1 Data Reconstruction . . . . . . . . . . . . . . . . 49

$4.1 .1 \quad \operatorname{PASS} \ldots \ldots \ldots \ldots \ldots \ldots \ldots$

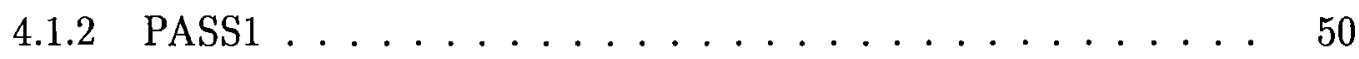

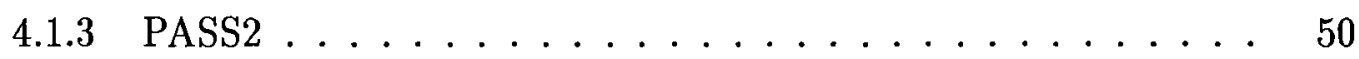

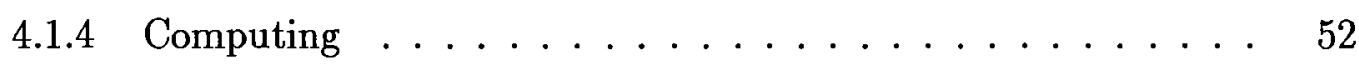

$4.2 \quad$ E769 Monte Carlo . . . . . . . . . . . . . . . . . 52

4.2 .1 Event Generation . . . . . . . . . . . . . . 53

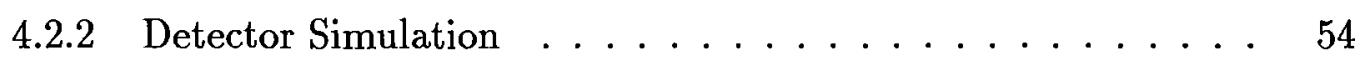

4.3 Signal Extraction $\ldots \ldots \ldots \ldots \ldots \ldots \ldots \ldots \ldots$

4.3 .1 Pair $\operatorname{Strip} \ldots \ldots \ldots \ldots \ldots \ldots \ldots \ldots$

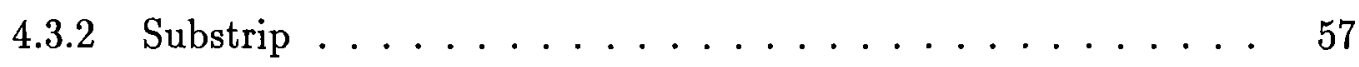

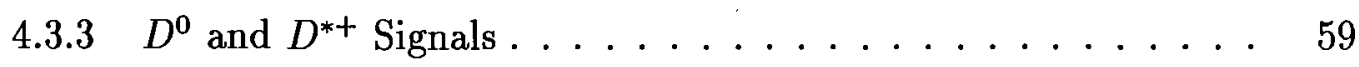

5 Results

5.1 Mass Distributions . . . . . . . . . . . . . . . 62

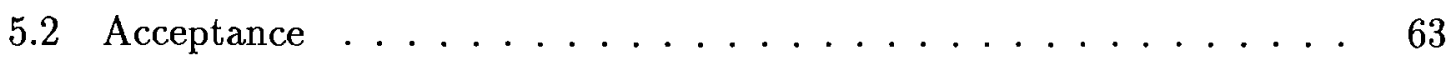

5.2 .1 Čerenkov Efficiency Correction $\ldots \ldots \ldots \ldots \ldots$ 
5.2 .2 Trigger Efficiency Correction . . . . . . . . . . . . 71

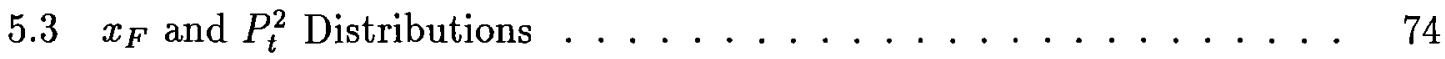

5.3.1 Leading Particle Effect . . . . . . . . . . . . . 77

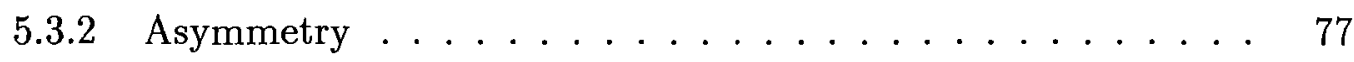

$5.3 .3 \quad$ Systematic Errors . . . . . . . . . . . . . . 78

6 Conclusion $\quad 80$

6.1 Comparison to Other Modes . . . . . . . . . . . . . . . 80

6.2 Comparison to Other Experiments . . . . . . . . . . 82

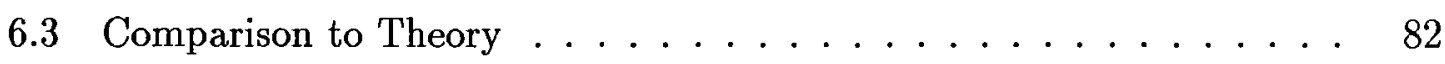

6.4 Summary $\ldots \ldots \ldots \ldots \ldots \ldots \ldots \ldots \ldots$

$\begin{array}{ll}\text { A Trigger Efficiency Functions } & 86\end{array}$

$\begin{array}{ll}\text { Bibliography } & 89\end{array}$

$\begin{array}{ll}\text { Biographical Sketch of the Author } & 92\end{array}$ 


\section{List of Tables}

1.1 Values of the $n$ and $b$ parameters for the inclusive $D$ production cross

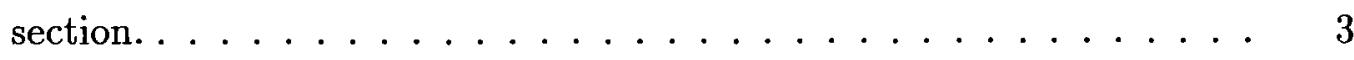

2.1 Quarks $\left(\operatorname{spin} \frac{1}{2}\right) \ldots \ldots \ldots \ldots$

3.1 Properties of the segmented target. . . . . . . . . . . . 23

3.2 E769 threshold $\check{C}$ counters characteristics. . . . . . . . . . . . . . 28

$3.3 \mathrm{C} 1$ and $\mathrm{C} 2$ states vs particle momentum . . . . . . . . . . 29

3.4 TRD efficiencies and contaminations . . . . . . . . . . . . 32

3.5 E769 SMD characteristics. . . . . . . . . . . . . 37

3.6 E769 DC system characteristics. . . . . . . . . . . . . . . 38

3.7 E769 magnets characteristics. . . . . . . . . . . . . . . 40

4.1 Pair strip cuts. . . . . . . . . . . . . . . . 56

4.2 Substrip cuts. . . . . . . . . . . . . . . . 59

4.3 Final analysis cuts. ....................... 60

5.1 Number of $D^{0}$ and $D^{*}$ events per $x_{F}$ bin. . . . . . . . . . 63

5.2 Number of $D^{0}$ and $D^{*}$ events per $P_{t}^{2}$ bin. . . . . . . . . . 63

5.3 Signal size and its relative value in the negative and positive runs for $D^{0}$ and $D^{*} \ldots \ldots \ldots \ldots \ldots \ldots$

5.4 The shape parameters of the differential cross section distributions for $D^{0}, D^{0}$ (daughter), and $D^{*} \ldots \ldots \ldots \ldots \ldots$ 
5.5 The shape parameters of the differential cross section distributions for the leading and the non-leading $D^{*}$ particles and for the corresponding

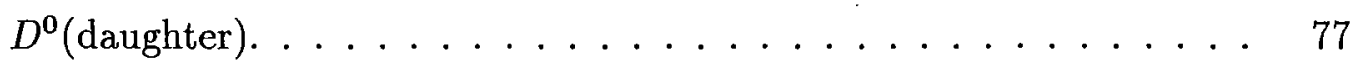

5.6 Fractional systematic errors of $n$ and $b$ for $D^{0}$ and $D^{*}$. . . . . . 78

6.1 Comparison of the production parameters of different $D^{0}$ data samples. 81

6.2 Comparison of the production parameters of different $D^{*}$ data samples. 81

6.3 Comparison of $D^{0}$ production parameters from experiments with inci-

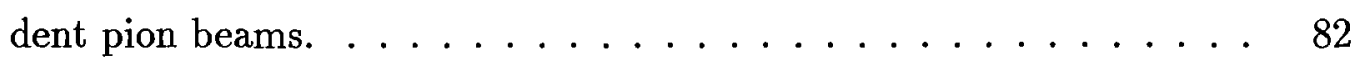

6.4 Comparison of $D^{*}$ production parameters from experiments with inci-

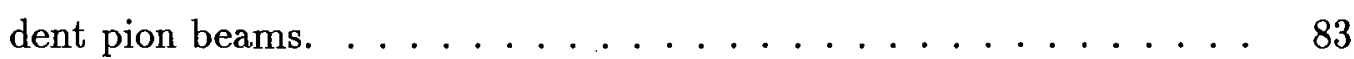

6.5 Comparison of $n$ parameters with the theoretical calculations. . . . 83

A.1 Fit parameters for $E_{t}$. OR. $E_{t B}$ trigger efficiency function. $\ldots \ldots 87$ 


\section{List of Figures}

2.1 The QCD picture of the hadroproduction of charm ....... 8

2.2 Parton structure functions for nucleons (DO1 set) . . . . . . . . 11

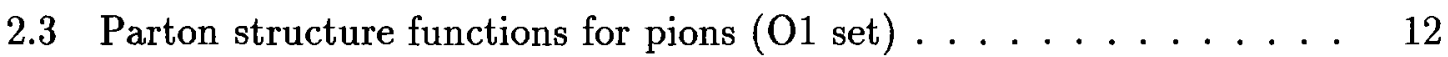

2.4 The graphs contributing to the lowest order $\left(\alpha_{s}^{2}\right)$ parton cross section 13

2.5 Examples of graphs contributing in order $\alpha_{s}^{3}$ to the cross section . . . 13

2.6 Differential cross section of charm production in $\pi p$ interactions versus $x_{F} \ldots \ldots \ldots \ldots \ldots \ldots \ldots \ldots$

2.7 (a) Lund and (b) Collins fragmentation functions $\ldots \ldots \ldots \ldots$

2.8 Differential cross section results for $D^{*}$ using different fragmentation

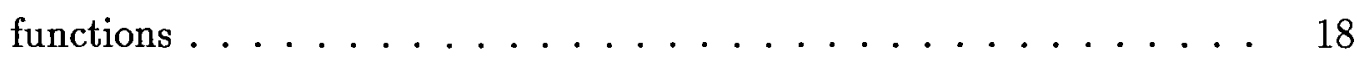

3.1 Schematic layout of beam acceleration at Fermilab . . . . . . . . 20

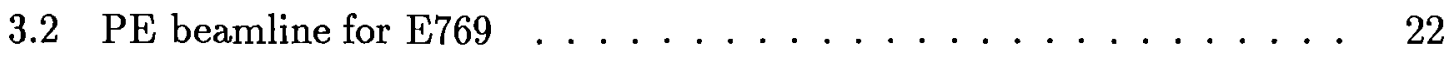

3.3 E769 Target Region . . . . . . . . . . . . . . . . . . . 23

3.4 Schematic view of the E769 detector system. . . . . . . . . . 25

3.5 Schematic of the DISC optical assembly. . . . . . . . . . . 26

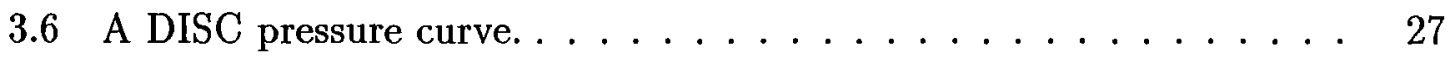

3.7 DISC PMT coincidences vs TRD plane counts. . . . . . . . . . 30

3.8 Schematic of a TRD radiator-chamber module. . . . . . . . . . . 31

3.9 TRD plane count distribution . . . . . . . . . . . . . . . 32

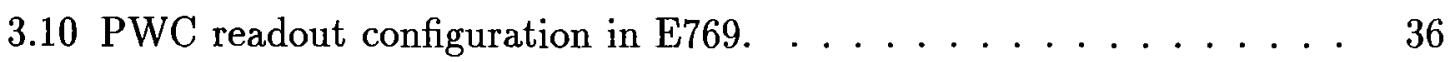

3.11 Cross Section of an SMD Plane . . . . . . . . . . . . . . 37

3.12 Orientation of $\mathrm{X}, \mathrm{U}$, and $\mathrm{V}$ Planes . . . . . . . . . . . . 39

3.13 SLIC radiator-scintillator assembly. . . . . . . . . . . . . . 42 
3.14 Schematic view of E769 calorimeter system. . . . . . . . . . . 42

3.15 Diagram of E769 trigger. . . . . . . . . . . . . . . . 45

3.16 Schematic of the E769 data acquisition system. . . . . . . . . 46

4.1 Schematic representation of the principal analysis cuts. . . . . . 58

$4.2 D^{0}$ and $D^{*}$ signals for the pion beam in $E_{t}$ or $E_{t B}$ triggers. . . . . 61

5.1 $D^{0}$ mass plots per $x_{F}$ bin. Plots (a) through (f) show the mass distributions in each $x_{F}$ bin of width 0.1 for the range $0.0-0.6 \ldots \ldots$. . 64

$5.2 D^{0}$ mass plots per $P_{t}^{2}$ bin. Plots (a) through (f) show the mass distributions in each $P_{t}^{2}$ bin of unit width for the range $0-6 \mathrm{GeV}^{2} / \mathrm{c}^{2}$. . .

5.3 $D^{0}$ acceptance plots for (a) the negative runs, (b) the positive runs, and (c) their average per $x_{F}$ bin. $\ldots \ldots \ldots \ldots \ldots \ldots$

$5.4 D^{0}$ acceptance plots for (a) the negative runs, (b) the positive runs, and (c) their average per $P_{t}^{2}$ bin. $\ldots \ldots \ldots \ldots 6$

$5.5 \quad D^{*}$ acceptance plots in bins of $x_{F}$ and $P_{t}^{2} \ldots \ldots \ldots \ldots \ldots$

5.6 Čerenkov kaon efficiency versus kaon momentum for data and MC. . 72

$5.7 E_{t}$. OR. $E_{t B}$ trigger efficiencies for negative and positive beams. . . . 73

5.8 Differential cross section distributions for (a) $D^{0}$ and (b) $D^{*}$ in bins of

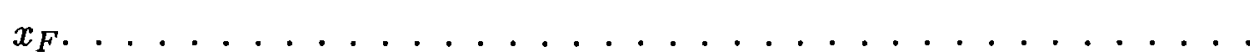

5.9 Differential cross section distributions for (a) $D^{0}$ and (b) $D^{*}$ in bins of

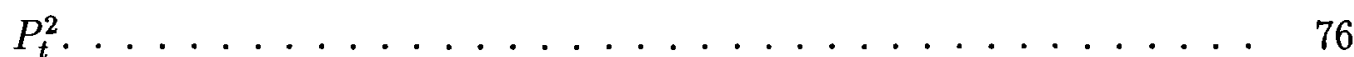

5.10 Differential asymmetry versus $x_{F}$ for the leading vs. non-leading sam-

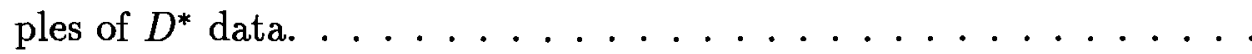

A.1 Efficiency vs $P_{t 715}$ for $E_{t}$. OR. $E_{t B}$ trigger in (a) $-210 \mathrm{GeV}$, (b) $-250 \mathrm{GeV}$, (c) $+250 \mathrm{GeV},(\mathrm{d})$ proton run regions. . . . . . . . . . . 


\section{Chapter 1}

\section{Introduction}

Charmed particles have been produced in electron-positron, neutrino-hadron, photonhadron and hadron-hadron interactions. In hadronic production of charm, however, the extraction of a clean signal has been relatively difficult due to the presence of a large background. If we were to express the charm cross section as a fraction of the total rate for different processes, it would be of order $1 ; 1 / 10 ; 1 / 100$ and $1 / 1000$ for $e^{-} e^{+}, \nu$-hadron, $\gamma$-hadron and hadron-hadron, respectively [1]. As a result, the progress in the understanding of the properties and mechanisms for the hadroproduction of charm has been relatively limited.

Experiment 769 at Fermilab was the first charm hadroproduction experiment that combined silicon microstrip tracking technology with a high rate data acquisition system to collect a very high statistics data sample. With this approach it was able to reconstruct more charm particles than had ever been seen before in hadron-nucleon collisions. In this dissertation, we use this unprecedented sample to study charmed meson production. Below, we give a brief account of the early experiments prior to Fermilab E769, and then discuss the E769 experiment itself.

\section{$1.1 \quad$ Early Experiments}

The early charm hadroproduction experiments have tried to measure such properties as the total cross section, the atomic mass dependence, the longitudinal and transverse 
momentum dependence of charmed particle production. In this section, we only discuss the early results for the differential cross section distributions since they are the focus of this dissertation.

The differential cross section dependence on longitudinal and transverse momentum is usually described in terms of the variables $x_{F}$, and $P_{t}^{2}$ in the following form:

$$
\frac{d^{2} \sigma}{d P_{t}^{2} d x_{F}} \propto\left(1-\left|x_{F}\right|\right)^{n} e^{-b P_{t}^{2}}
$$

where $x_{F} \simeq 2 P_{z}^{*} / \sqrt{s}$, and $P_{t}=\sqrt{P_{x}^{2}+P_{y}^{2}} \cdot P_{z}^{*}$ is the momentum of the particle along the direction of the incident beam in the center of mass frame, and $s$ is the total center of mass energy squared. In table 1.1, we summarize the measured values of the parameters $n$ and $b$ for some of the experiments reviewed by Tavernier in reference [1]. All parameters quoted are the results of a global fit to all $D$ mesons.

An important observation from the above results is the dependence of the parameter $n$ on the nature of the final state charmed particles. NA27 results indicate different $x_{F}$ distributions for the $D$ mesons which can contain a valence quark from the $\pi^{-}$beam (leading) and those which cannot contain such a valence quark (nonleading). These experimental $x_{F}$ distributions are important in the understanding of the charm production mechanism in hadron-hadron interactions.

Experiment NA11, unlike NA27, does not confirm the large difference between the distributions of the leading and the non-leading sample events. However, the limited acceptance in the crucial region of $0.0<x_{F}<0.2$, makes it difficult for NA11 to provide a conclusive evidence againt the leading effect.

This effect was not predicted by the quantum chromodynamics (QCD) parton model, and its proof would suggest a recombination mechanism where the charm quark recombines with one of the valence quarks in the incident hadron. The implications of such an effect, and the existence of the confusing results among other things, have prompted more recent experiments with higher statistics to try to address such issues with better accuracy. 


\begin{tabular}{|c|c|c|c|c|}
\hline Expt & Beam/Target & $\begin{array}{l}\text { Beam Momentum } \\
(\mathrm{GeV} / \mathrm{c})\end{array}$ & $\mathrm{n}$ & $\begin{array}{c}\mathrm{b} \\
(\mathrm{GeV} / \mathrm{c})^{-2}\end{array}$ \\
\hline $\begin{array}{l}\text { NA16 } \\
(1983)\end{array}$ & $\pi^{-} / p$ & 360 & $\begin{array}{c}2.8 \pm 0.8 \\
\left(x_{F}>0.0\right) \\
\\
2.0 \pm 1.0 \\
\text { (leading) } \\
\\
6.0 \pm 3.0 \\
\text { (non-leading) }\end{array}$ & $1.1 \pm 0.3$ \\
\hline $\begin{array}{l}\text { NA27 } \\
(1985)\end{array}$ & $\pi^{-} / p$ & 360 & $\begin{array}{c}3.8 \pm 0.6 \\
\left(x_{F}>0.0\right) \\
\\
1.8 \pm_{0.5}^{0.6} \\
\text { (leading) } \\
\\
7.9 \pm 1.6 \\
\text { (non-leading) }\end{array}$ & $1.18 \pm_{0.16}^{0.18}$ \\
\hline $\begin{array}{l}\text { NA11 } \\
(1986)\end{array}$ & $\pi^{-} / B e$ & 200 & $\begin{array}{c}1.5 \pm_{0.8}^{1.0} \\
\left(x_{F}>0.2\right) \\
\\
1.1 \pm_{0.5}^{0.6} \\
\text { (leading) } \\
\\
1.5 \pm_{0.8}^{1.0} \\
\text { (non-leading) }\end{array}$ & $0.9 \pm 0.2$ \\
\hline
\end{tabular}

Table 1.1: Values of the $n$ and $b$ parameters for the inclusive $D$ production cross section. 


\section{$1.2 \quad$ Fermilab E769}

The small size of the data collected by the early charm hadroproduction experiments (100 events or less), and the large uncertainties associated with their results motivated more recent experiments to gather much larger samples of data. Since 1988, there has been a steady progress on both the experimental and the theoretical fronts in the understanding of the hadroproduction of charm. Experiments at CERN and Fermilab have resulted in cleaner data of higher statistical precision. Theoretical calculations of the higher order contributions to charm hadroproduction in QCD have enhanced the confidence in the ability of the present theory to interpret the experimental data. For a progress report on the recent developments, refer to the review article by J.A. Appel on the hadroproduction of charm particles [2].

One of the recent experiments with higher statistics is E769. Experiment 769 was performed during the 1987-88 fixed target run in the Tagged Photon Lab (TPL) at Fermilab, using a mixed hadron beam $(\pi, K, p)$ on a segmented target of four different elements $(B e, A l, C u, W)$. Employing a nearly open trigger and a relatively fast data acquisition system, E769 was able to collect approximately $370 \times 10^{6}$ events. E769 was designed to study the hadroproduction properties including the total cross section, beam flavor dependence, atomic number dependence, $x_{F}$ and $P_{t}$ dependences.

This thesis describes the study of the differential cross section distributions of $D^{0} \rightarrow K^{-} \pi^{+} \pi^{-} \pi^{+}$and $D^{*+} \rightarrow D^{0}\left(K^{-} \pi^{+} \pi^{-} \pi^{+}\right) \pi^{+}$decay modes and their charge conjugates using the data from E769. Combining the results of these modes with those of other modes obtained by E769, we will compare them with the results of more recent experiments. 


\section{Chapter 2}

\section{Theory}

The hadroproduction of charm has been one of the more complex processes to grapple with theoretically; however, a good deal of progress has been made toward its understanding in recent years. This chapter will give an overview of the conventional theory used in explaining the recent experimental results.

First, we present a brief historical background to the standard model, then an introduction to the theory behind the interaction of hadrons, and finally we will review the main components in the calculation of the cross section for the hadroproduction of charm.

\subsection{Quarks}

In 1964, Gell-Mann and Zweig suggested that all hadrons were composed of elementary constituents which Gell-Mann named "quarks" [3, 4]. According to this model, hadrons are composed of three quarks (baryon), or three antiquarks (antibaryon), or a quark and an antiquark (meson).

At the time, only three quarks were proposed to exist. However, later that year, Bjorken and Glashow postulated the existence of a fourth quark they called "charm" [5]. The fourth quark was re-introduced in 1970 by Glashow, Iliopoulos, and Maiani (GIM) to explain the low branching ratio for $K_{L}^{0} \rightarrow \mu^{+} \mu^{-}$by cancelling the contributions of the strangeness-changing neutral currents [6]. But, direct experimental 


\begin{tabular}{|c||c|c|c|c|c|c|}
\hline Flavor & $u$ & $d$ & $s$ & $c$ & $b$ & $t$ \\
\hline \hline Charge & $+\frac{2}{3}$ & $-\frac{1}{3}$ & $-\frac{1}{3}$ & $+\frac{2}{3}$ & $-\frac{1}{3}$ & $+\frac{2}{3}$ \\
\hline
\end{tabular}

Table 2.1: Quarks (spin $\frac{1}{2}$ )

evidence for charm was not observed until 1974 when C. C. Ting's group at the Brookhaven National Laboratory (BNL) and Burton Richter's group at the Stanford Linear Accelerator Center (SLAC) each discovered the $J / \psi(c \bar{c})$ meson independently $[7,8]$.

Today, five of six flavors of the quarks postulated by the standard model have been observed, and the search for the sixth $(t)$ is ongoing (Table 1.1). Besides spin and charge, quarks were found to possess another property or quantum number called "color" which constitutes the basis for the mechanism of quark-quark interaction described in the following section.

\subsection{Quantum Chromodynamics (QCD)}

The origin of the development of QCD seems to derive from a theoretical objection to the early quark model which violated the exclusion principle. According to this principle, no two fermions (particles of half-integer spin) can occupy the same quantum state. This implies that no two quarks (spin 1/2) can have the same quantum numbers in an ensemble. However, we know that $\Delta^{++}(u u u)$ is supposed to consist of three identical $u$ quarks in the same state. In 1964, O. W. Greenberg put forward a solution; the addition of a new quantum number, called color [9]. Each quark can have one of the three colors: red $(R)$, green $(G)$, or blue $(B)$.

One of the experimental confirmations of the color quantum number lies in the measurement of the quantity $R$, where

$$
R \equiv \frac{\sigma\left(e^{-} e^{+} \rightarrow \text { hadrons }\right)}{\sigma\left(e^{-} e^{+} \rightarrow \mu^{-} \mu^{+}\right)}=N_{c} \sum_{q} e_{q}^{2} .
$$


$N_{c}$ is the number of colors for each quark flavor, $q$ is the quark flavor, and $e$ is the charge of the quark. All measurements of $R$ point to a value of 3 for $N_{c}$.

An interesting feature of the color concept is that all real particles are "colorless" or "white"; i.e., they are combinations of a color and an anticolor (e.g., $R \bar{R})$ in case of a meson, or of all three colors $(R G B)$ as for a baryon.

Just as the electromagntic force between charged particles is mediated by photons, the strong force between quarks is mediated by the exchange of a virtual "gluon". Gluons are bicolored, consisting of a color and an anticolor (e.g. $R \bar{G}$ ).

The experimental evidence for the existence of gluons was obtained by the measurement of the quark distribution functions from deep inelastic scattering (DIS) off protons and neutrons. For example, in the case of proton, it has been calculated that only $54 \%$ of its momentum is carried off by the quarks; the rest is carried by the neutral gluons.

In quantum electrodynamics (QED), the photons as field quanta do not interact with each other since they do not carry electric charge. Unlike photons, gluons in quantum chromodynamics can directly interact with each other because they have color charge. One of the implications of gluon-gluon interaction is the "antiscreening" of the color charge (just the opposite of charge screening effect in QED), which turns out to be the factor in the viability of the QCD theory. The gluon-gluon interactions (or gluons turning into pairs of gluons) reverse the familiar result of the vacuum polarization in QED: a red charge, for example, is preferentially surrounded by other red charges [10]. In other words, the farther away one gets from a (bare) color charge, the stronger it seems. This makes the asymptotic behavior much different from that in QED.

This is evident in the formula for the coupling constant in QCD:

$$
\alpha_{s}\left(Q^{2}\right)=\frac{12 \pi}{\left(11 N_{c}-2 N_{f}\right) \ln \left(Q^{2} / \Lambda^{2}\right)}
$$

where $Q$ is the momentum transfer between the partons, $N_{c}$ is the number of colors ( 3 , in the Standard Model), $N_{f}$ is the number of flavors (6, in the Standard Model), and $\Lambda$ is the QCD mass scale. 


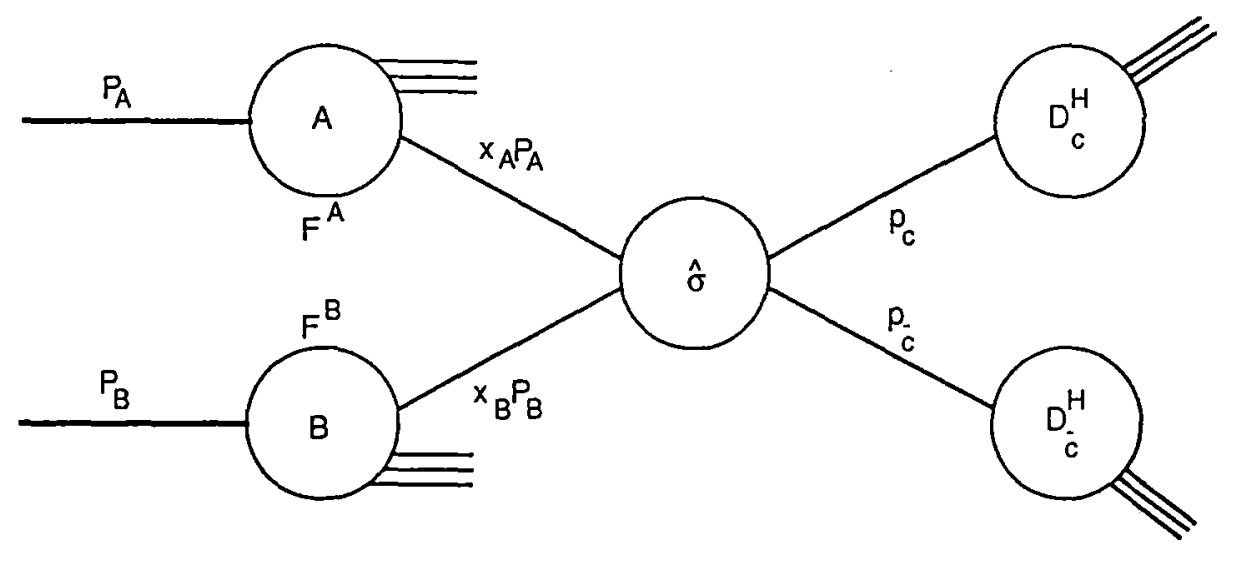

Figure 2.1: The QCD picture of the hadroproduction of charm

In strong interactions with large momentum transfer (short distances), the coupling constant $\left(\alpha_{s}\right)$ is relatively small $(\sim 0.2)$ and we can apply standard perturbative techniques. However, for small $Q^{2}$ (large distances) the coupling constant can be $\sim 1$, which makes the use of the same techniques problematic. In other words, at short distances, the "strong" force between quarks is relatively weak and they can be treated as essentially free quarks. This subsequently leads to asymptotic freedom required by Bjorken scaling which in turn allows the use of the Feynman calculus in QCD.

\subsection{Charm Cross Section}

The hadroproduction of charm as understood by QCD is depicted schematically in Figure 2.1. The production process is shown as a hard scattering of two partons, one from each incident hadron, denoted by $\hat{\sigma}$. The partons carry a fraction $x$ of the hadron's momentum, and have a structure function (or probability density) of $F$. To calculate the charm pair $(c \bar{c})$ cross section we need to know $F$ and $\hat{\sigma}$. However, in order to compare theory with our experimental results, we need to calculate the 
cross section for charm hadrons since quarks fragment into hadrons. This process is represented by the fragmentation function $D_{c}^{H}$.

The charm pair $(c \bar{c})$ differential cross section with respect to Feynman- $x$ is therefore formulated as:

$$
\frac{d \sigma}{d \hat{x}_{F}}=\sum_{i, j} \int d x_{A} d x_{B}\left[\frac{d \hat{\sigma}_{i j}\left(x_{A} P_{A}, x_{B} P_{B}, \hat{p}, m_{c}, Q^{2}\right)}{d \hat{x}_{F}}\right] F_{i}^{A}\left(x_{A}, Q^{2}\right) F_{j}^{B}\left(x_{B}, Q^{2}\right) .
$$

$\hat{p}$ and $m_{c}$ are the momentum and the mass of the charm quark. $Q$ is the typical momentum transfer between the parton and the charm. The Feynman- $x$ variable, $\hat{x}_{F}$, is defined as:

$$
\hat{x}_{F}=\frac{\hat{p}_{z}}{p_{\max }} \simeq \frac{2 \hat{p}_{z}}{\sqrt{s}}
$$

where $\hat{p}_{z}$ is the momentum of the charm quark in the direction of the incident hadron, and $\sqrt{s}$ is the center of mass energy.

The cross section calculations for hadroproduction of charm can be divided into three main parts: structure functions, parton-parton cross section, and fragmentation functions. The following subsections will deal with each of these parts in more detail.

\subsubsection{Structure Functions}

As mentioned above, to calculate the charm cross section, one needs to know the structure functions of the incident particles. In our case, we need to have the parton distribution functions for pions and nucleons (protons and neutrons). To determine how these functions are calculated, we refer to Duke and Owens parameterization [11] for nucleons, and Owens parameterization [12] for pions.

In these parameterization methods, the structure functions are estimated by performing fits to the data on deep-inelastic lepton-nucleon scattering, high mass dilepton production, and the production of massive particles such as $J / \psi$. The fitting program operates by directly integrating the Altarelli-Parisi equations in $x$ space, but for convenience, the results are parameterized in terms of $s=\ln \left[\ln \left(Q^{2} / \Lambda^{2}\right) / \ln \left(Q_{0}^{2} / \Lambda^{2}\right)\right]$. The distribution functions are specified at $Q_{0}^{2}=4(\mathrm{GeV} / \mathrm{c})^{2}$, and subsequently evolved to higher values of $Q^{2}$ using the Altarelli-Parisi equations ${ }^{1}$. With no exception, all

\footnotetext{
${ }^{1}$ At this input value of $Q^{2}$, the charm sea quark distribution in nucleon (or pion) is assumed to be zero $[11,12]$.
} 
structure functions must satisfy the following relation due to the conservation of momentum:

$$
\sum_{i} \int x F_{i}(x) d x=1
$$

where $i$ signifies all the partons.

Duke and Owens provide two sets of distributions, set 1 for $\Lambda=200 \mathrm{MeV}$, and set 2 for $\Lambda=400 \mathrm{MeV}$. For the sake of demonstration, we choose set 1 because of its better agreement with the data. Figures 2.2 and 2.3 show the distribution functions of valence quarks $x V(x)$, gluons $x G(x)$, and sea quarks $x S(x)$, in nucleons and pions respectively. All distributions have been plotted for $Q^{2}=Q_{0}^{2}=4(\mathrm{GeV} / \mathrm{c})^{2}$; i.e., $s=0$.

\subsubsection{Parton-Parton Cross Section}

The parton short distance cross section $\hat{\sigma}$ is calculated as a power series expansion in $\alpha_{s}$ using perturbative techniques in QCD. Nason, Dawson, and Ellis have developed an analytic expression for the parton differential cross section up to order $\alpha_{s}^{3}$ [13].

The subprocesses which contribute to the lowest order $\left(\alpha_{s}^{2}\right)$ parton cross section are to large extent gluon-gluon fusion, and to a lesser degree quark-antiquark annihilation. The representative Feynman diagrams of these processes are shown in figure 2.4. Figure 2.5 shows some of the graphs that contribute to the gluon-gluon initiated process in order $\left(\alpha_{s}^{3}\right)$. The top graphs are examples of virtual emission diagrams, and the bottom ones are real emission diagrams. It must be added however that the inclusion of the first radiative correction $\left(\alpha_{s}^{3}\right)$ does not appreciably alter the shapes of the lowest order distributions.

There is a small charge asymmetry in the production of heavy quarks due to the interference terms in the $q \bar{q}$ annihilation subprocess. This effect is more pronounced for larger $x_{F}$. Figure 2.6 shows the $x_{F}$ distributions of $c$ and $\bar{c}$ production in $\pi^{-} p$ collisions.

To derive these distributions, Nason, Dawson, and Ellis use the parton distribution functions of Owens (set 1) [12] for the pion, and of DFLM [14] for the proton. They use the scale $Q^{2}=4\left(m_{c}^{2}+P_{t}^{2}\right)$ where $m_{c}=1.5 \mathrm{GeV} / \mathrm{c}^{2}$, and $P_{t}$ is the transverse 


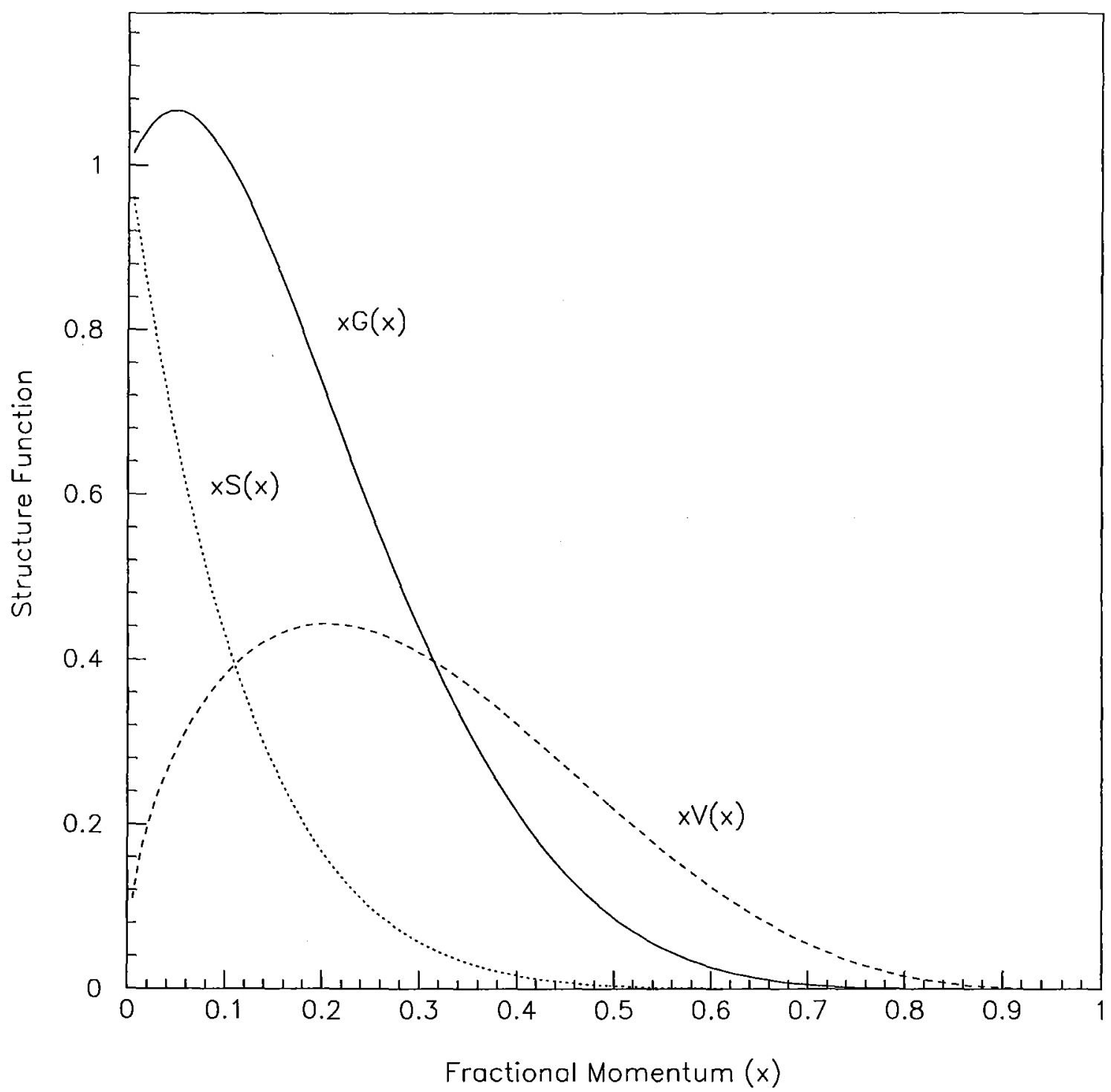

Figure 2.2: Parton structure functions for nucleons (DO1 set) 


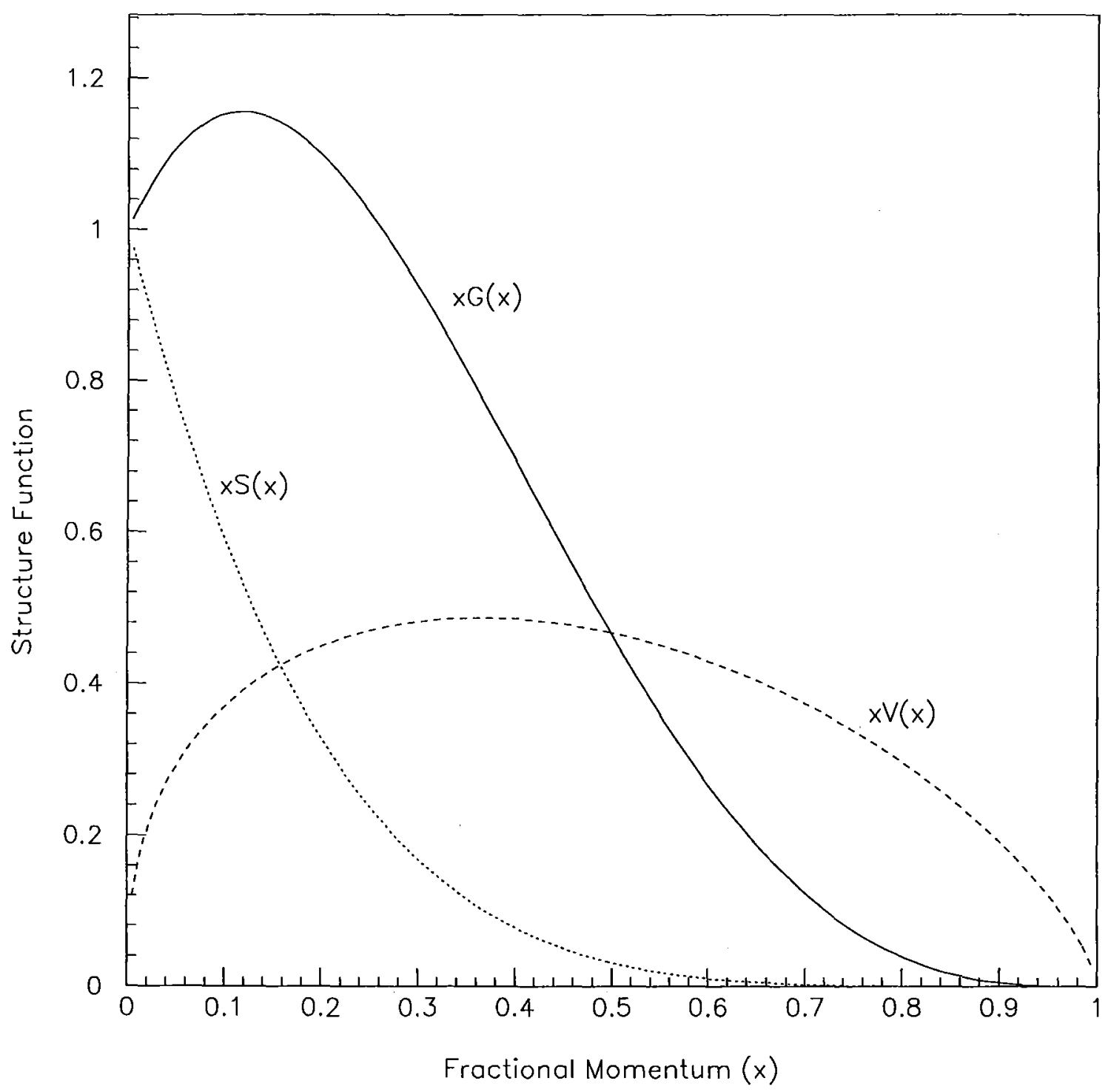

Figure 2.3: Parton structure functions for pions (O1 set) 


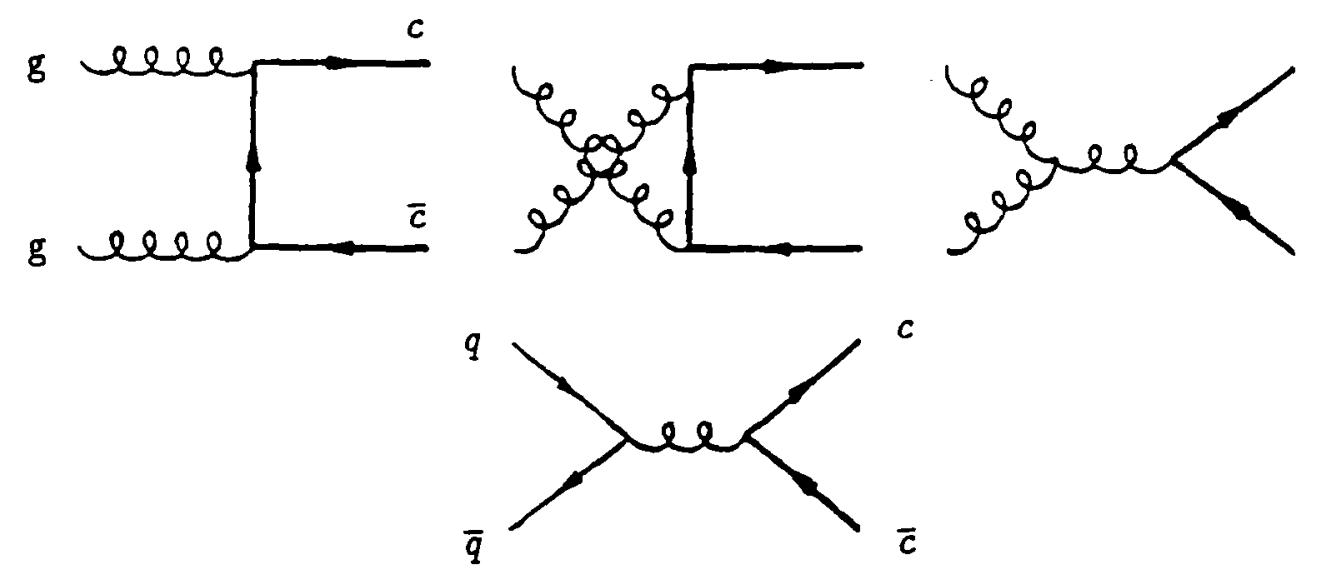

Figure 2.4: The graphs contributing to the lowest order $\left(\alpha_{s}^{2}\right)$ parton cross section
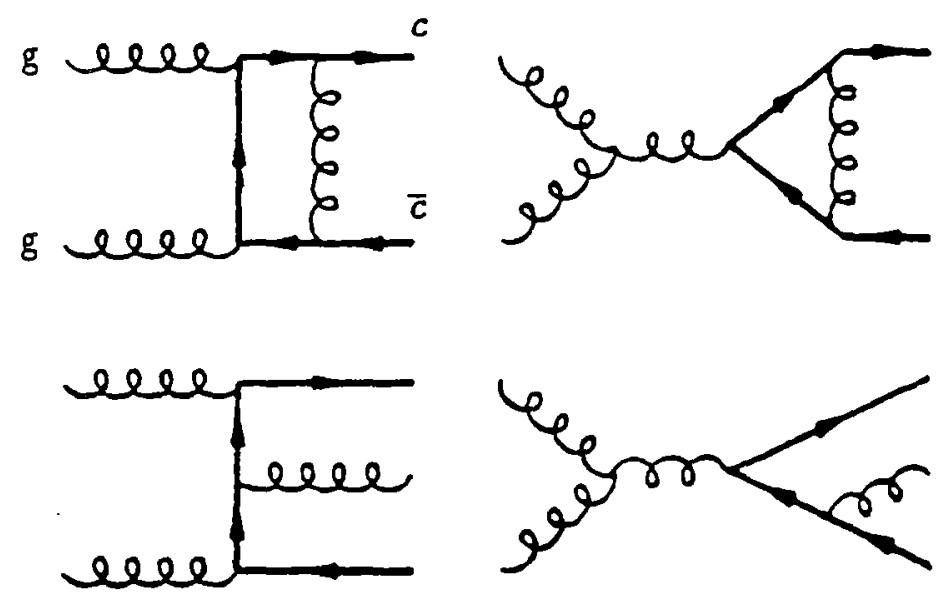

Figure 2.5: Examples of graphs contributing in order $\alpha_{s}^{3}$ to the cross section 


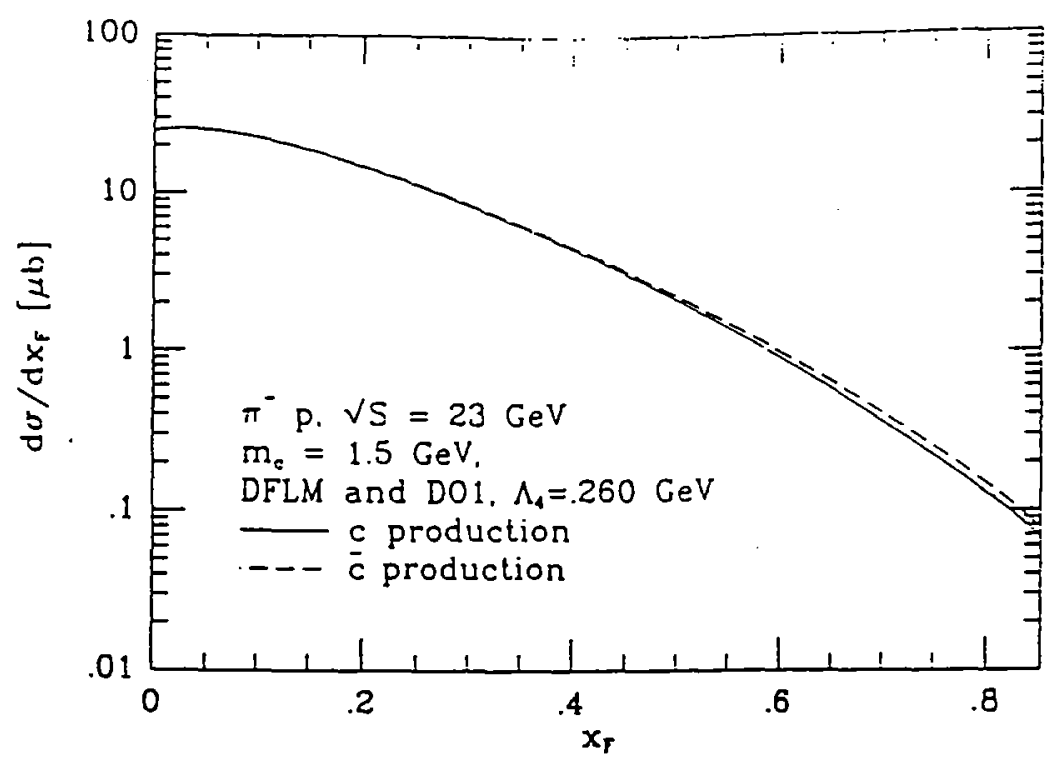

Figure 2.6: Differential cross section of charm production in $\pi p$ interactions versus $x_{F}$

momentum of the produced charm quark.

There are three major sources of uncertainties in the parton cross section calculations. Firstly, there is the uncertainty on the value of $\Lambda$ which affects the coupling constant $\alpha_{s}$. Then, there is the uncertainty on the mass of the charm quark and the choice of scale. Lastly, there are the uncalculated effects of even higher order perturbative corrections which can contribute to the uncertainties in our predictions.

\subsubsection{Fragmentation Functions}

The hadronization process through which partons are transformed into hadrons is dominated by color-confining forces $\left(\alpha_{s}>1\right)$, and consequently requires a nonperturbative approach in the development of a QCD model. Presently, there are three dominant fragmentation models: Independent Fragmentation (IF), Cluster Fragmentation (CF), and String Fragmentation (SF). These models are reviewed in [15]. In all of these, the produced heavy quark $(Q)$ gradually loses energy through the production of $(q \bar{q})$ pair. This process continues until there is not enough energy for the 
pair production from the sea. At this point, the heavy quark gets color-confined with the light quarks to form a hadron.

Analytical formulas for the fragmentation function have been derived in the context of the string model [16]. The fragmentation function $\left(D_{q}^{h}(z)\right)$ represents the probability of producing a hadron $(h)$ with fractional momentum $(z)$ of quark $(q)$. It describes the transition (parton $\rightarrow$ hadron) just as the structure function, $F(x)$, describes the embedding (hadron $\rightarrow$ parton). And like $F$ functions (eq. 2.5), $D$ functions are subject to constraints imposed by the conservation of momentum [10]:

$$
\sum_{h} \int_{0}^{1} z D_{q}^{h}(z) d z=1
$$

Of the formulas for the fragmentation function discussed in [16], we choose to plot those of Andersson et al. and of Collins and Spiller. We chose Andersson's because of its close agreement with the data, and Collins' because of its inclusion of the reciprocity rule ${ }^{2}$ and the effect of the transverse motion[16].

Lund symmetric function (Andersson et al.):

$$
D_{Q}^{H}\left(x^{+}\right)=N \frac{\left(1-x^{+}\right)^{\beta}}{x^{+}} \exp \left[\frac{-B m_{H \perp}^{2}}{x^{+}}\right]
$$

$x^{+}=\frac{E+P_{\|}}{E_{\max }+P_{\max }}$, and $m_{H \perp}=\sqrt{m_{H}^{2}+P_{t}^{2}}$.

Collins and Spiller:

$$
D_{Q}^{H}(x)=N\left[\frac{1-x}{x}+\frac{2-x}{1-x} \epsilon_{Q}\right]\left(1+x^{2}\right)\left[1-\frac{1}{x}-\frac{\epsilon_{Q}}{1-x}\right]^{-2}
$$

$x=P / P_{\max }$, and $\epsilon_{Q}=\left[\frac{\left\langle P_{t}^{2}\right\rangle}{m_{Q}^{2}}\right]$.

The values of $\beta$ and $B$ in equation 2.7 , and $\epsilon_{Q}$ in equation 2.8 were selected from the fits to the data for $D^{*}$ in [16], and are as follows: $\beta=1.02, B=0.43\left(\mathrm{GeV} / \mathrm{c}^{2}\right)^{-2}$, and $\epsilon_{Q}=0.64$. Figure 2.7 shows the two functions for these fitted parameters.

\footnotetext{
${ }^{2}$ The reciprocity rule states that, for $x \rightarrow 1$, the fragmentation function of a heavy quark into a hadron should equal the structure function of that in that hadron.
} 


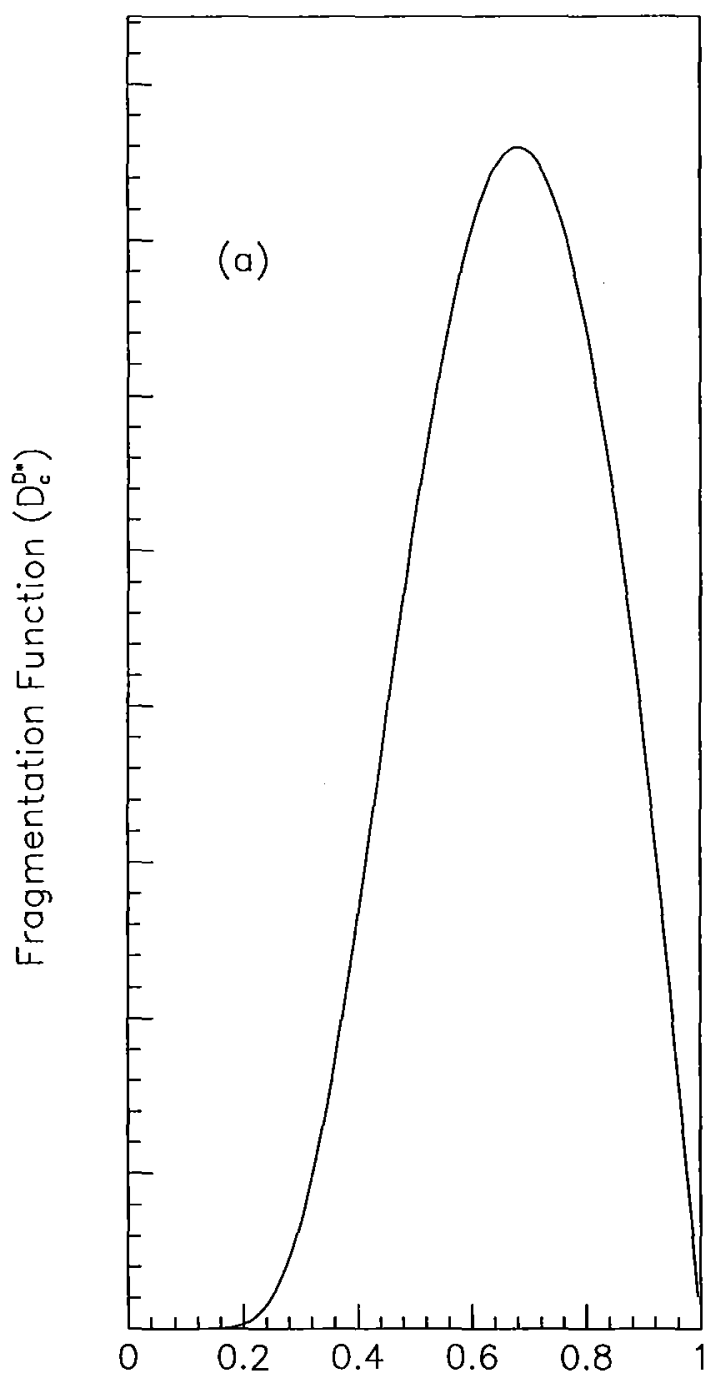

Fractional Momentum $\left(\mathrm{x}^{+}\right)$

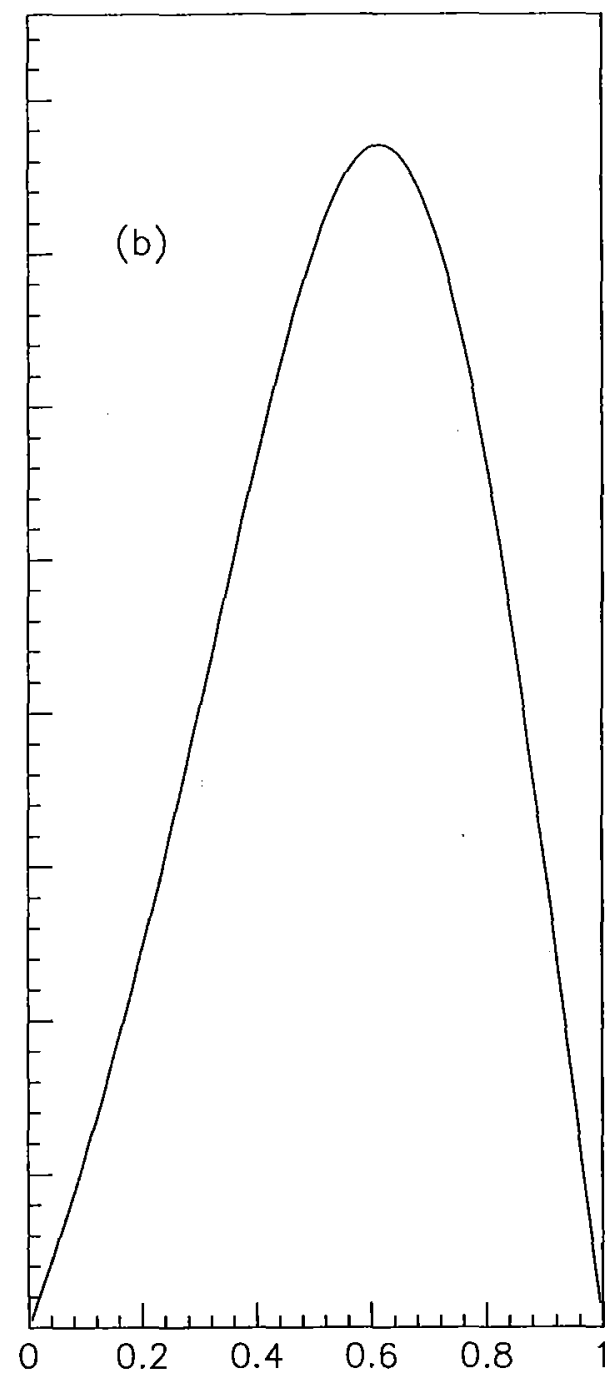

Fractional Momentum (x)

Figure 2.7: (a) Lund and (b) Collins fragmentation functions 


\subsection{4 $x_{F}$ Distribution of Charm Hadron Cross Section}

Now that we basically have all the components for the calculation of the differential cross section of a charm hadron, we can formulate it in a way that is easy to parameterize. Starting with the charm quark cross section, we can state that the number of charm quarks produced in the momentum range $(\hat{P}, \hat{P}+d \hat{P})$ is proportional to $d \hat{\sigma}(\hat{P})$. The probability that these charm quarks fragment into charm hadrons in the momentum range $(P, P+d P)$ is:

$$
D_{c}^{H}\left(\frac{P}{\hat{P}}\right) d\left(\frac{P}{\hat{P}}\right)=D_{c}^{H}\left(\frac{P}{\hat{P}}\right) \frac{d P}{\hat{P}}
$$

and consequently the number of these hadrons in the above range is proportional to:

$$
d \sigma(P)=d \hat{\sigma}(\hat{P}) D_{c}^{H}\left(\frac{P}{\hat{P}}\right) \frac{d P}{\hat{P}}
$$

To calculate the differential cross section for the charm hadron in the full momentum range of the charm quark, we integrate equation 2.10 :

$$
\frac{d \sigma(P)}{d P}=\int_{P}^{\hat{P}_{\max }} \frac{d \hat{\sigma}(\hat{P})}{d \hat{P}} \frac{d \hat{P}}{\hat{P}} D_{c}^{H}\left(\frac{P}{\hat{P}}\right)
$$

Defining $\hat{x}=P / \hat{P}$ and $x=P / P_{\max }$, we can rewrite equation 2.11 as:

$$
\frac{d \sigma(x)}{d x}=\frac{1}{x} \int_{x}^{1} \frac{d \hat{\sigma}(\hat{x})}{d \hat{x}} D_{c}^{H}(\hat{x}) \hat{x} d \hat{x}
$$

We can easily replace the $x$ variable with $x_{F}$ without altering our results. For the charm cross section component of the integral, $d \hat{\sigma} / d \hat{x}$, we use NDE's distribution (Fig. 2.6) parameterized with the functional form of $(1-x)^{n}$. For the fragmentation functions, we use the two functions plotted in figure 2.7 (Andersson et al., and Collins \& Spiller). The results of the numerical integration of equation 2.12 for the above functions are plotted in figure 2.8. In Andersson's fragmentation function, we assume an average transverse momentum $\left(P_{t}\right)$ of $1 \mathrm{GeV} / \mathrm{c}$ for $D^{*}$. All three distributions are normalized to the same value in their first data point, and the fit is to the points with $x_{F}>0.2$ since it is in this range that $x$, and $x^{+}$can be safely replaced by $x_{F}$. 


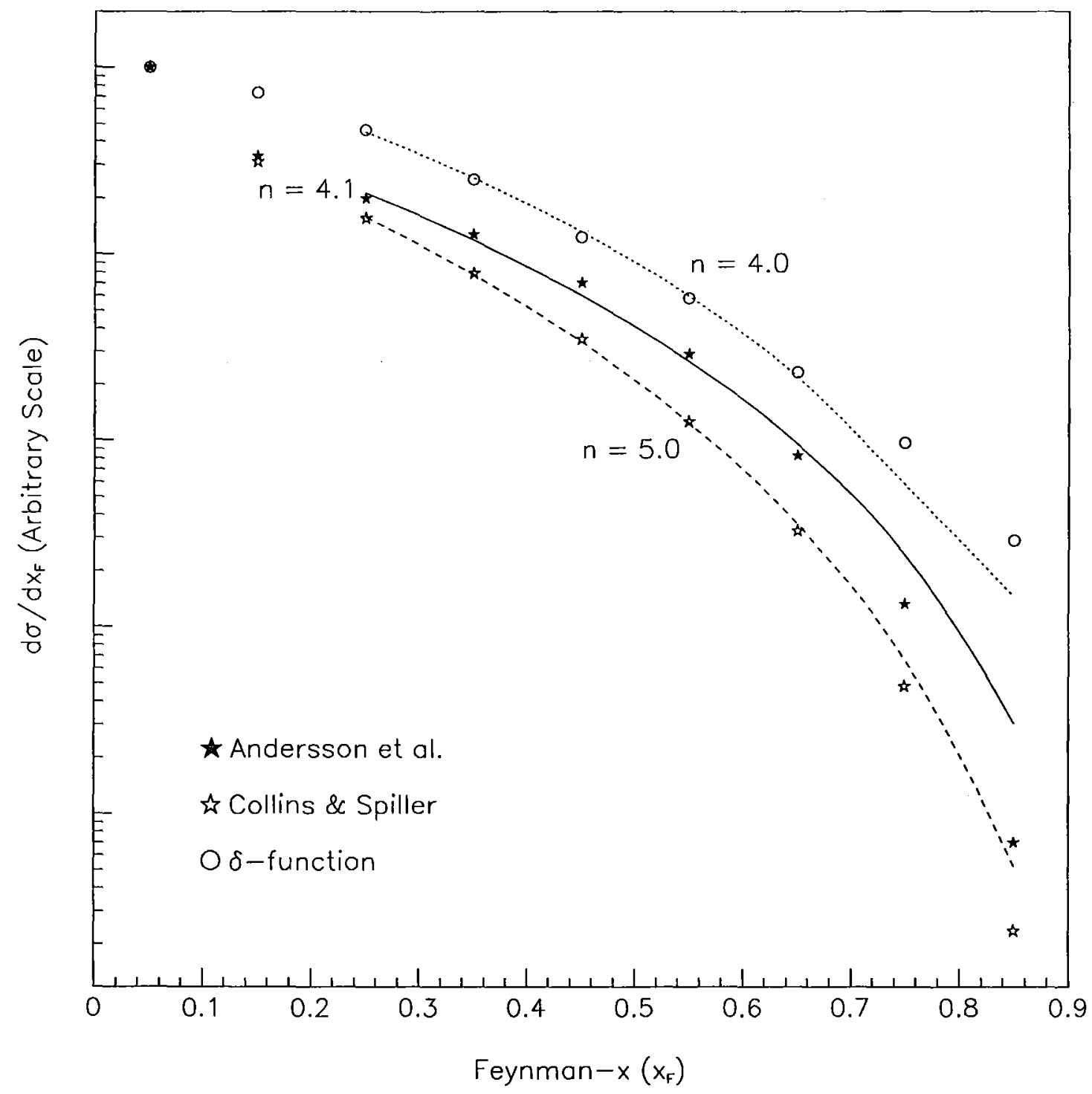

Figure 2.8: Differential cross section results for $D^{*}$ using different fragmentation functions 


\section{Chapter 3}

\section{Experiment}

This chapter deals solely with the various components of the experiment in general. The first section gives a description of how the primary proton and the secondary hadron beams are produced. The following section briefly discusses the target, while the third section gives a more detailed description of the detector system. Next, we review the triggers before discussing the data acquisition system.

\subsection{Beam}

E769 used a $250 \mathrm{GeV}$ hadron beam consisting of charged pions $\left(\pi^{\mp}\right)$, kaons $\left(K^{\mp}\right)$ and protons $(p)$. The choice of a mixed beam was to allow the study of the beam flavor dependence of charm hadroproduction.

This section deals only with the production of the hadron beam. The identification and tracking of the beam are discussed in the detector section.

\subsubsection{Primary Proton Beam}

The accelerator at Fermilab is a proton accelerator. The production of protons starts with the dissociation of the hydrogen molecules $\left(\mathrm{H}_{2}\right)$ into atoms $(\mathrm{H})$ using radio frequency (rf) energy [17]. Then the hydrogen atoms are passed over a cesium (Cs) source where each atom picks up an electron. The $H^{-}$ions are subsequently accelerated by the Cockcroft-Walton accelerator and injected into the linear accelerator 


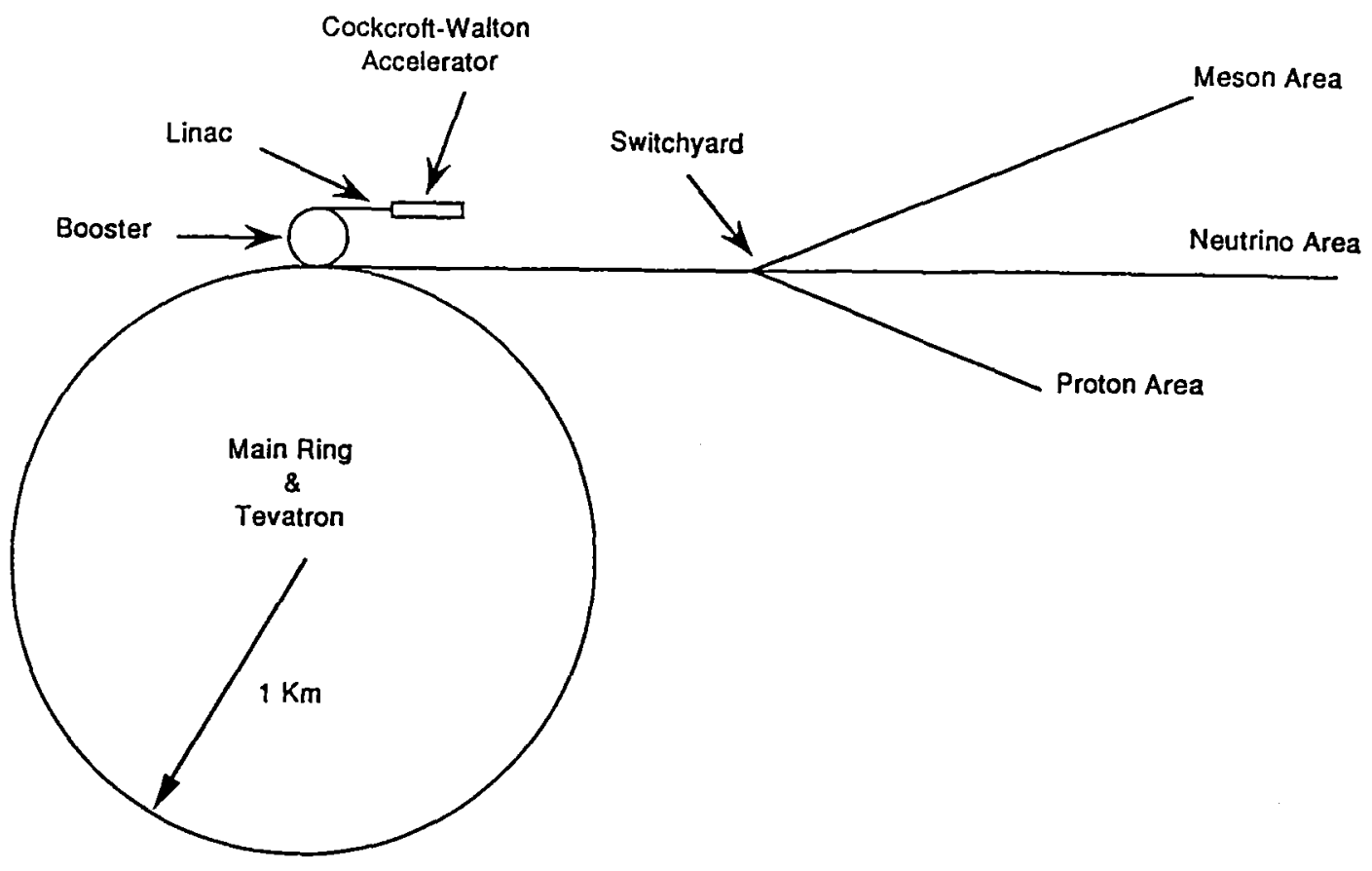

Figure 3.1: Schematic layout of beam acceleration at Fermilab

(LINAC) at an energy of $750 \mathrm{KeV}$. The rf cavities in the LINAC accelerate the ions to $200 \mathrm{MeV}$ and simultaneously bunch them into buckets about 2 ns long and 19 ns apart. The $H^{-}$ions are stripped of their electrons when passing through a thin carbon foil at the entrance of the booster ring.

The booster stores the LINAC output pluse and accelerates it to $8 \mathrm{GeV}$ in 1/15 sec. A series of booster pulses are injected into the main ring before the acceleration cycle begins. The protons are then accelerated to $150 \mathrm{GeV}$ by going through rf cavities which increase their energies by $2.8 \mathrm{MeV}$ per crossing [17].

The final stage of acceleration is achieved in the Tevatron where protons reach their highest energy of $800 \mathrm{GeV}$. About $1 \times 10^{13}$ protons are extracted during a period of 22 seconds (called spill). Next, the protons are sent to the switchyard to be split into three separate beams for the three experimental areas (Fig. 3.1).

\subsubsection{Secondary Hadron Beam}

The beam at the proton area is split into three beamlines: Proton-West (PW), ProtonCenter (PC), and Proton-East (PE). The Tagged Photon Laboratory (TPL) where 
E769 was performed is located in the PE beamline.

In $\mathrm{PE}$, the typical beam flux was $1.2 \times 10^{12}$ protons/spill. There, $800 \mathrm{GeV}$ protons were directed at a primary target of $30 \mathrm{~cm}$ thick beryllium where upon interaction different particles with a wide range of momenta were produced. From these particles, a secondary beam of $250 \mathrm{GeV}$, with an energy spread of only $\pm 0.2 \%$ [18], was selected by using bending magnets and collimators. The composition of this hadron beam was $93 \% \pi^{-}, 5.2 \% K^{-}$, and $1.5 \% \bar{p}$ in the negative beam, and $61 \% \pi^{+}, 34 \% p$, and $4.4 \%$ $K^{+}$in the positive beam.

The hadron beam was then directed through a collimator with 3 interchangeable holes controlled by the experimenter at the TPL control console. With the aid of this collimator and the following two quadrupole magnets, the experimenter could align the beam to go through the beam tagging system. The typical flux at this point was $4 \times 10^{7}$ per spill.

Figure 3.2 shows the PE beam transport system comprised mainly of magnetic dipoles and quadrupoles used for bending and focusing the beam. The beam tagging system consisted of a differential Čerenkov counter (DISC), and a transition radiation detector (TRD). For tracking the beam, we used two sets of multiwire proportional chambers (MWPC) and silicon microstrip detectors (SMD). These detectors are discussed in more detail in section 2.3 .

\subsection{Target}

The choice of targets is usually based on considerations such as the interaction rate and the multiple scattering effect. As a result, one tries to choose a target that is thick enough to produce the desirable number of events for the given beam rate, but not too thick to cause a large number of multiple scatterings or secondary interactions.

Taking the above considerations into account, E769 employed a segmented target of 26 foils of four types of material: beryllium $(B e)$, aluminum $(A l)$, copper $(C u)$, and tungsten $(W)$. Targets of different atomic mass (A) were chosen to study the A-dependence of charm production. The simultaneous use of all four materials was a factor in the reduction of the systematic errors due to changing conditions of the 

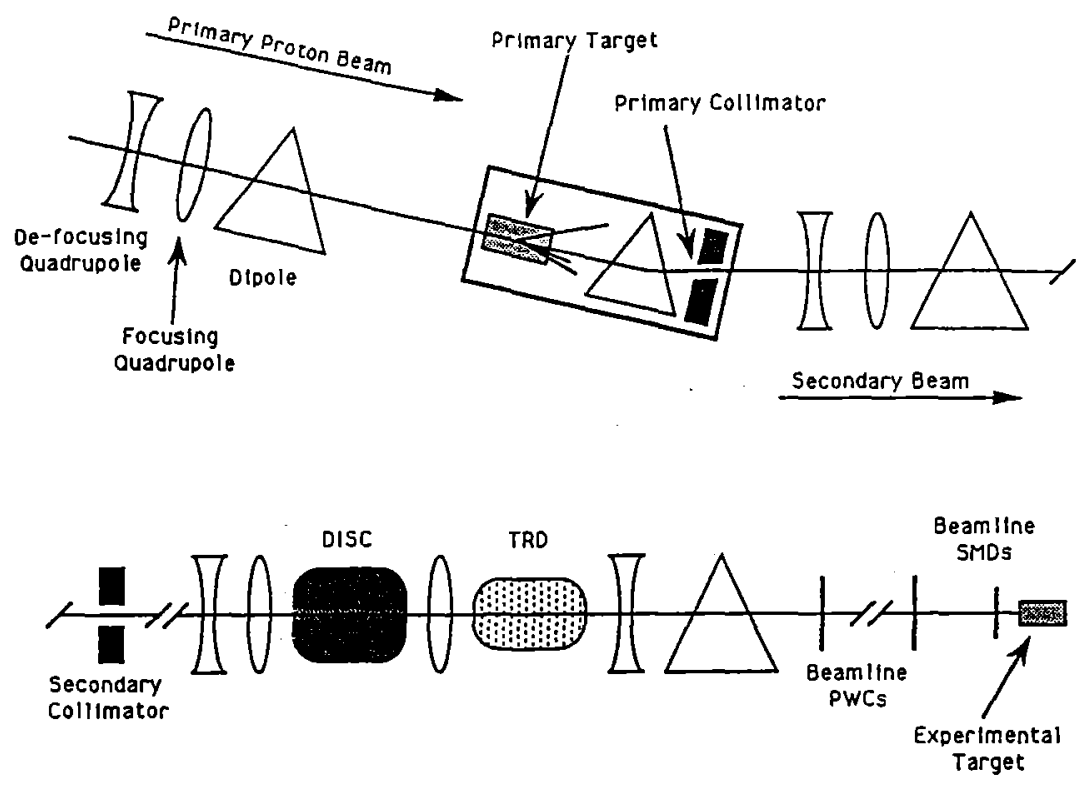

Figure 3.2: PE beamline for E769

runs [19]. The foils were about $250 \mu \mathrm{m}$ thick each, except for tungsten foils which were about $100 \mu \mathrm{m}$ thick, and all were more than $1 \mathrm{~mm}$ apart.

The order in which different target types were placed was to minimize the effect of multiple scattering in the downstream foils. Since the net angle of deflection depends on the radiation length of the target according to equation 3.1 [20], we place the material with the shortest radiation length $\left(X_{0}\right)$ the most upstream.

$$
\phi_{r m s}=\left\langle\phi^{2}\right\rangle^{1 / 2} \propto \sqrt{\frac{t}{X_{0}}}
$$

where $t$ is the thickness of the target. Table 3.1 lists some of the properties of our target material [21]. The numbers in parentheses are percentages of the radiation and interaction lengths corresponding to each target material thickness.

Figure 3.3 shows the target region where the segmented target is placed between the beam spot and the halo counters upstream and the interaction counter downstream. The function of the counters is explained in the trigger section of this chapter. 


\begin{tabular}{|c|c|c|c|c|c|}
\hline Material & A & $\begin{array}{c}\text { total thickness } \\
\mathrm{t} \\
\mathrm{cm}\end{array}$ & $\begin{array}{c}\text { density } \\
\rho \\
\mathrm{g} / \mathrm{cm}^{3}\end{array}$ & $\begin{array}{c}\text { Radiation length } \\
X_{0} \\
\mathrm{~g} / \mathrm{cm}^{2}(\%)\end{array}$ & $\begin{array}{c}\text { Interaction length } \\
\lambda_{I} \\
\mathrm{~g} / \mathrm{cm}^{2}(\%)\end{array}$ \\
\hline$B e$ & 9 & 0.2604 & 1.848 & $65.19(0.74 \%)$ & $75.2(0.64 \%)$ \\
\hline$A l$ & 27 & 0.1260 & 2.70 & $24.01(1.42 \%)$ & $106.4(0.32 \%)$ \\
\hline$C u$ & 64 & 0.0761 & 8.96 & $12.86(5.30 \%)$ & $134.9(0.51 \%)$ \\
\hline$W$ & 184 & 0.0383 & 19.3 & $6.76(10.93 \%)$ & $185(0.40 \%)$ \\
\hline
\end{tabular}

Table 3.1: Properties of the segmented target.

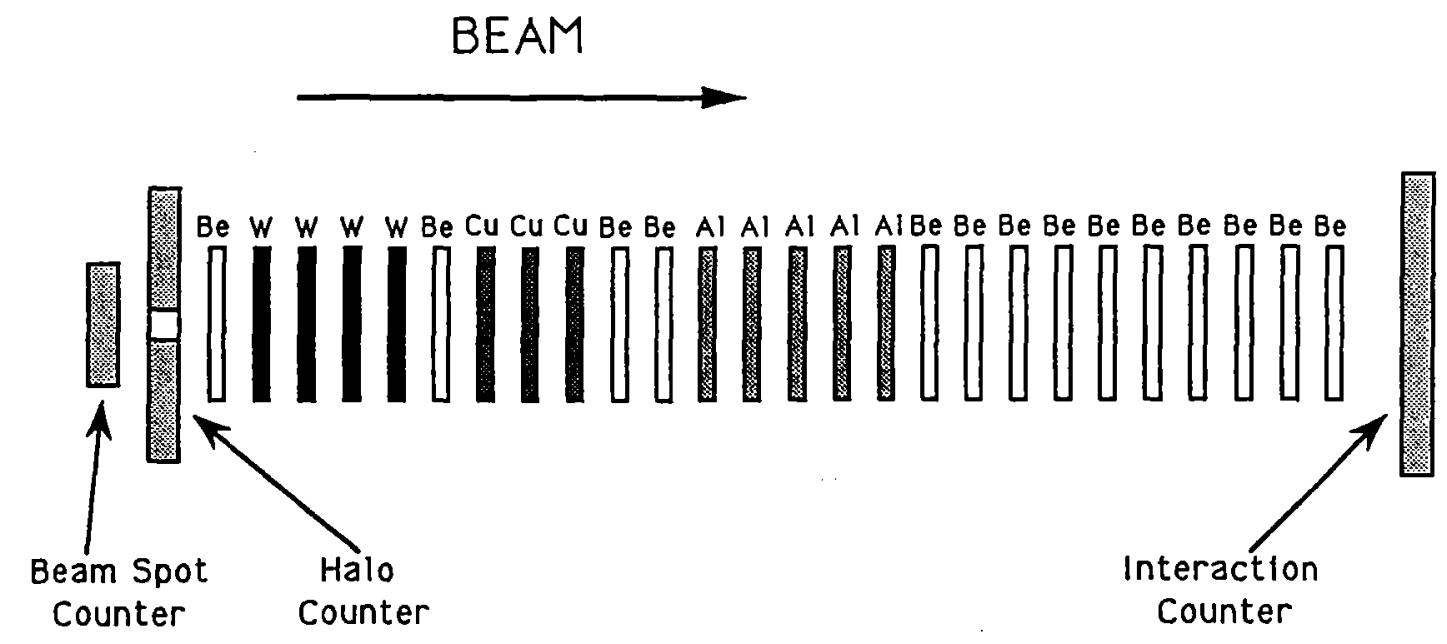

Figure 3.3: E769 Target Region 


\subsection{E769 Detector System}

The E769 detector system consisted of the following components: a differential Čerenkov counter and a transition radiation detector for beam tagging, 8 multiwire proportional chamber planes and 2 silicon microstrip planes for beam tracking, 11 SMD planes, 2 MWPCs, 35 drift chamber planes and 2 analysis magnets for tracking of the interaction particles and measuring their momenta, 2 threshold Čerenkov counters for particle identification, an electromagnetic and a hadronic calorimeter for energy measurements, and a muon detector. Figure 3.4 shows the schematic view of the detector system. The following subsections describe each of these components separately.

\subsection{1 Čerenkov Counters}

The use of Cerenkov counters for particle ID is based on the phenomenon that a charged particle traversing a dielectric medium faster than the speed of light in that medium induces radiation. This effect occurs due to the polarization of the excited atoms in the vicinity of the charged particle, and their coherent emission of radiation at a characteristic angle $\theta$.

$$
\cos \theta=\frac{1}{\beta n}
$$

As evident from the above formula, to detect a measurable angle of $\theta$ in a medium with small index of refraction $(n)$, one needs to have fast moving particles. Accordingly, gas radiators are particularly useful for detecting particles with $\beta>0.99$.

The index of refraction of a gas can be changed by changing its pressure. Assuming an ideal gas, the relation between the two parameters is as follows:

$$
n-1=\left(n_{0}-1\right) \frac{P}{P_{0}}
$$

where the subscript 0 usually indicates the value of the parameter at atmospheric pressure.

There are basically two types of Čerenkov counters: threshold and differential counters. While the threshold Čerenkov counters simply detect particles whose velocities exceed some minimum limit $\left(\beta_{t}=1 / n\right)$, the differential Čerenkov counters can measure the velocity of the particles within a certain range. 


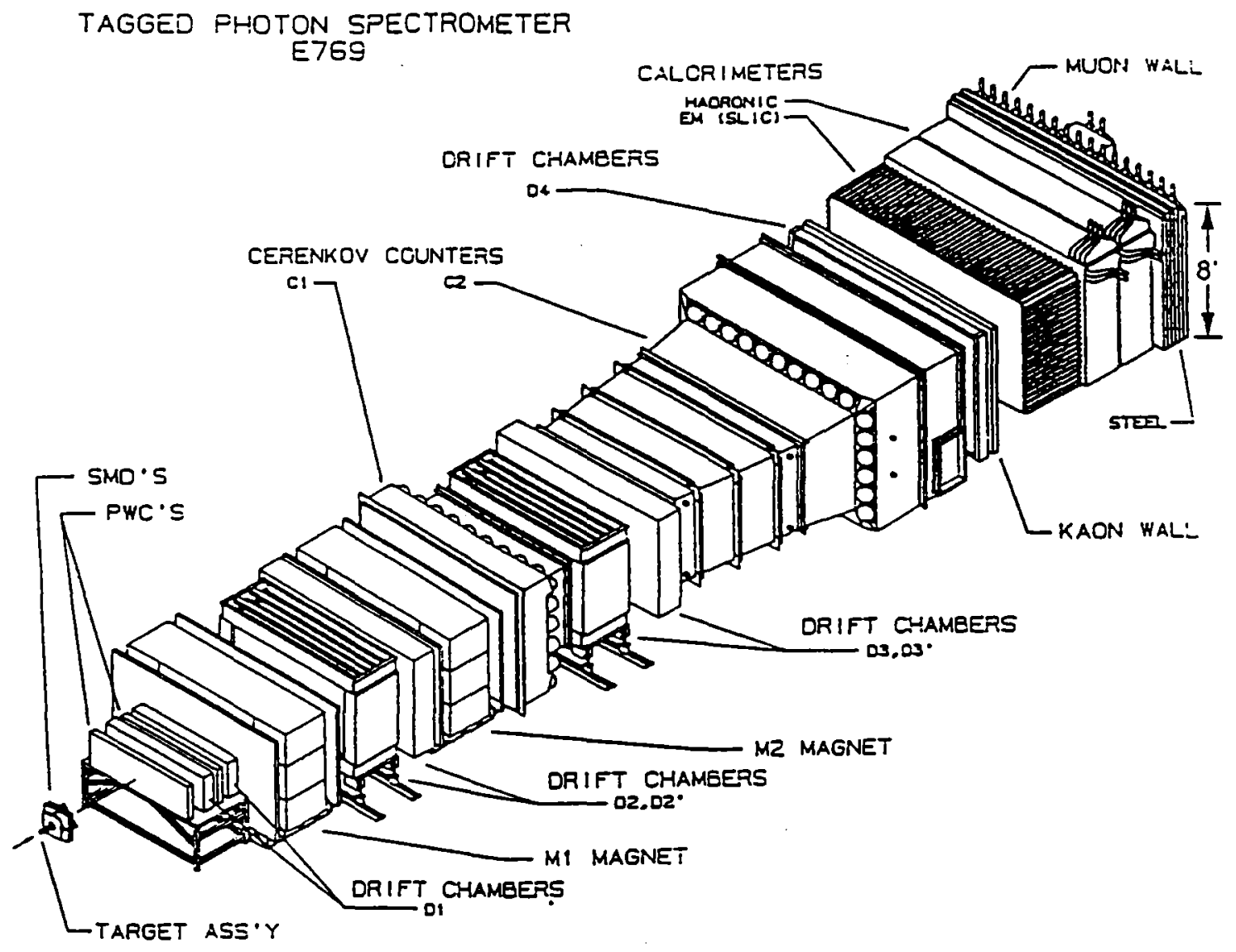

Figure 3.4: Schematic view of the E769 detector system. 


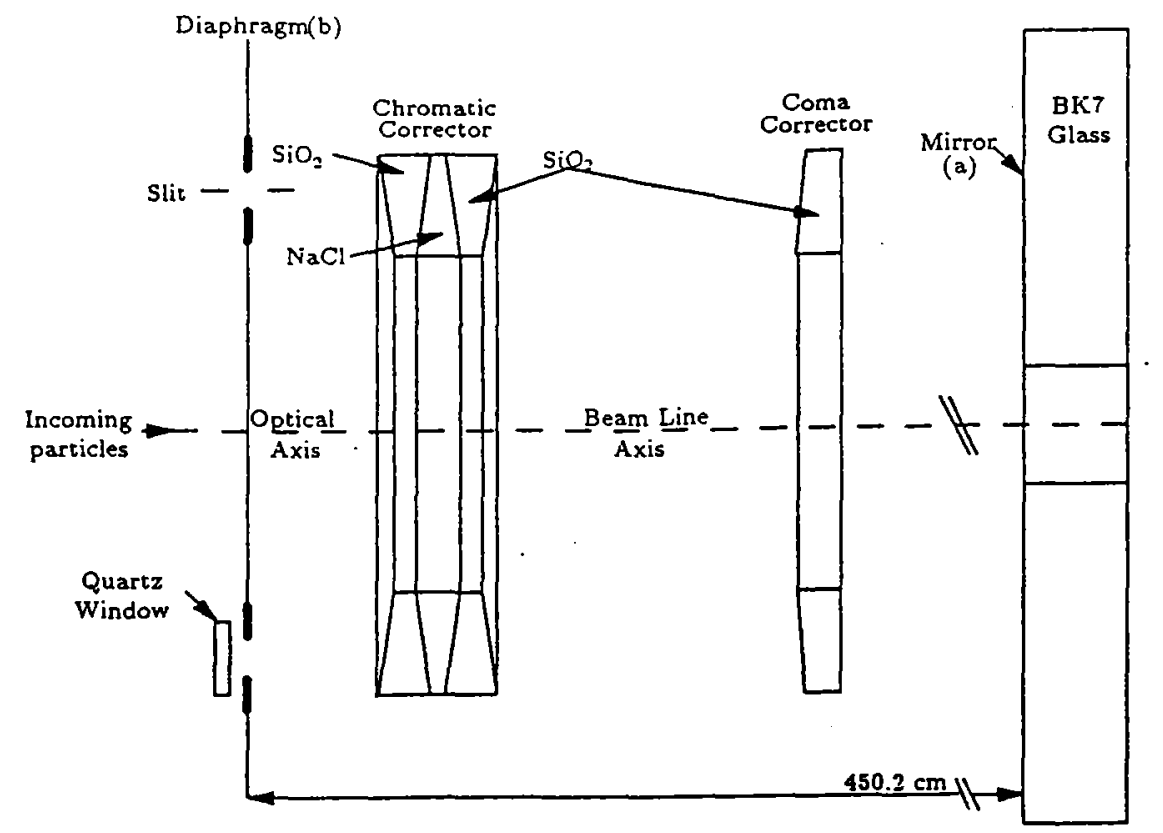

Figure 3.5: Schematic of the DISC optical assembly.

\section{Differential Counter}

Using this type of counter, one can detect the presence of particles of a given mass. E769 used the Differential Isochronous Self-Collimating Čerenkov Counter (DISC) for tagging the kaons in the mixed hadron beam. This device was originally designed and built at CERN in 1971 for use in the beamline of the experiment E96 at Fermilab. The DISC was filled with $H e$ gas ( $n=1.000035$ at STP) to a maximum pressure of $8.8 \mathrm{~atm}$. The pressure and temperature were monitored regularly so as to determine any significant changes affecting the index of refraction for Helium.

The DISC was designed to accept the Cerenkov light emitted at an angle of 24.5 mrad with respect to the beam line axis. This counter used an optical system to correct for dispersion and geometric aberrations (Fig. 3.5). The chromatic corrector was for the dispersion, and the coma corrector was for the spherical aberration in the mirror.

Differential counters are generally designed in such a way that the velcity resolution is less than half the velocity separation between the closest mass particles it is designed to detect. At $250 \mathrm{GeV}$, the difference in velocity between kaons and 


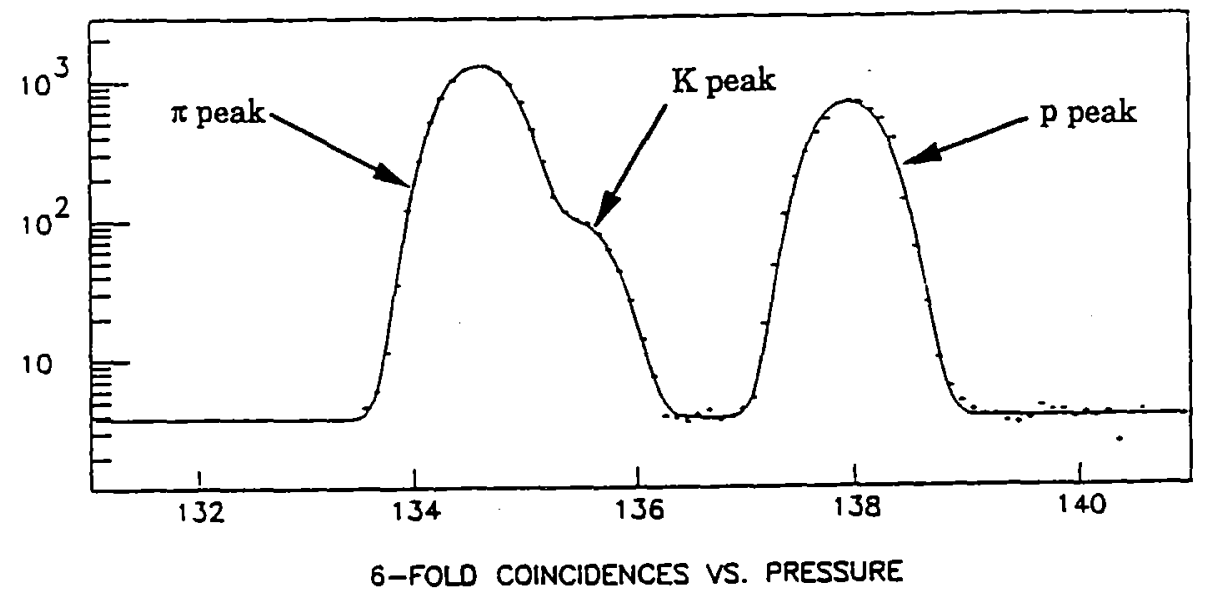

Figure 3.6: A DISC pressure curve.

pions is $\sim 3.6 \times 10^{-6} \mathrm{c}$, and the difference in the Cerenkov angle between them is $\sim 75 \mu \mathrm{rad}$. Our DISC had an angular resolution of $\sim 20 \mu \mathrm{rad}$ corresponding to a velocity resolution of $4 \times 10^{-7} \mathrm{c}$.

To calculate the efficiency of the DISC, pressure curves were periodically taken during the interspill. An on-line program incrementally increased the the pressure in the DISC and recorded the number of DICS photomultiplier tube (PMT) coincidences [18]. Figure 3.6 shows one of these curves. The pressure was usually set slightly higher than that for the kaon peak to reduce the pion contamination. The fits to these curves and the pressure and temperature information, along with the knowledge of the beam composition, were used to calculate the efficiency for correctly tagging kaons, and the contamination of the tagged sample by pions. The typical tagging efficiency turned out to be about $50 \%$, and the contamination about $1 \%$.

\section{Threshold Counter}

E769 used two threshold Čerenkov counters ( $\mathrm{C} 1$ and $\mathrm{C} 2$, Fig. 3.1) for particle identification. Some of the characteristics of these counters are listed in table 3.2.

As the table indicates, each counter was filled with a gas of different index of 


\begin{tabular}{|c|c|c|c|c|c|}
\hline Counter & Gas & $\begin{array}{c}\text { Index of } \\
\text { Refraction (n) }\end{array}$ & $\begin{array}{c}\pi \text { Threshold } \\
(\mathrm{GeV})\end{array}$ & $\begin{array}{c}K \text { Threshold } \\
(\mathrm{GeV})\end{array}$ & $\begin{array}{c}p \text { Threshold } \\
(\mathrm{GeV})\end{array}$ \\
\hline \hline $\mathrm{C} 1$ & $\mathrm{~N}_{2}$ & 1.000297 & 5.726 & 20.253 & 38.495 \\
\hline $\mathrm{C} 2$ & $\begin{array}{c}80 \% \mathrm{He} \\
20 \% \mathrm{~N}_{2}\end{array}$ & 1.0000874 & 10.556 & 37.337 & 70.966 \\
\hline
\end{tabular}

Table 3.2: E769 threshold Č counters characteristics.

refraction so that the threshold momentum for the particles would be different in each counter. The threshold momentum for a particle of mass $m$ is given by:

$$
p_{t h}=\frac{m \beta_{t}}{\sqrt{1-\beta_{t}^{2}}}
$$

Replacing $\beta_{t}$ with $1 / n$ gives:

$$
p_{t h}=\frac{m}{\sqrt{n^{2}-1}}
$$

Since $n \approx 1$, we can write $n$ as $1+\eta$ where $\eta \ll 1$. Rewriting equation 3.5 in terms of $\eta$ gives:

$$
p_{t h}=\frac{m}{\sqrt{\eta^{2}+2 \eta}} \approx \frac{m}{\sqrt{2 \eta}}
$$

By using $\mathrm{C} 1$ and $\mathrm{C} 2$ in tandem, one can develop a particle identification scheme shown in table 3.3. The table shows the state of the two theshold counters for each particle type in various momentum ranges, where $C$ indicates Čerenkov light was emitted by the particle, and $\bar{C}$ means it was not.

The Čerenkov light, collected by arrays of spherical mirrors, was reflected through conical surface structures known as Winston cones onto the phototubes [22]. The photomultiplier tubes (PMT) were RCA8854 5-inch tubes. The output signal of the PMTs were sent to LeCroy 2249 analog to digital converters (ADC) for digitization.

To calculate the particle identification probabilities for a particular track of momentum $P$, we determined the number of photo-electrons produced at a PMT, and predicted the number expected at the PMT due to the particle's momentum for different masses. Using this information, we calculated the probabilities for different particle types. When calculating the probabilities was not possible under certain circumstances, such as the absence of information due to physical complications or for 


\begin{tabular}{|c|l|l|l|}
\hline Momentum $(\mathrm{GeV} / \mathrm{c})$ & pion & kaon & proton \\
\hline \hline $0-6$ & $\bar{C}_{1} \bar{C}_{2}$ & $\bar{C}_{1} \bar{C}_{2}$ & $\bar{C}_{1} \bar{C}_{2}$ \\
\hline $6-11$ & $C_{1} \bar{C}_{2}$ & $\bar{C}_{1} \bar{C}_{2}$ & $\bar{C}_{1} \bar{C}_{2}$ \\
\hline $11-20$ & $C_{1} C_{2}$ & $\bar{C}_{1} \bar{C}_{2}$ & $\bar{C}_{1} \bar{C}_{2}$ \\
\hline $20-37$ & $C_{1} C_{2}$ & $C_{1} \bar{C}_{2}$ & $\bar{C}_{1} \bar{C}_{2}$ \\
\hline $37-38$ & $C_{1} C_{2}$ & $C_{1} C_{2}$ & $\bar{C}_{1} \bar{C}_{2}$ \\
\hline $38-71$ & $C_{1} C_{2}$ & $C_{1} C_{2}$ & $C_{1} \bar{C}_{2}$ \\
\hline $71+$ & $C_{1} C_{2}$ & $C_{1} C_{2}$ & $C_{1} C_{2}$ \\
\hline
\end{tabular}

Table 3.3: $\mathrm{C} 1$ and $\mathrm{C} 2$ states vs particle momentum

the momentum range of $0-6 \mathrm{GeV} / \mathrm{c}$, we assigned a priori values to the probabilities. These are typical probabilities for particles produced in hadronic interactions (independent of momentum). The a priori probabilities for different particle types are as follows: electron (0.02), muon (0.01), pion (0.81), kaon (0.12), and proton (0.04).

\subsubsection{Transition Radiation Detector (TRD)}

Transition radiation occurs when a charged particle crosses the interface between media with different dielectric or magnetic properties. This is due to the sudden redistribution of charges in the medium associated with the changing electric field of the particle. The radiation intensity is proportional to the Lorentz factor, $\gamma$, which enables one to differentiate among different particle types at relativistic energies.

Transition radiation detectors had already been used to tag final state electrons and hadrons. E769 was the first experiment to use TRDs for tagging high energy hadrons in incident beam [23]. We used our TRD to separate pions from protons and from kaons not tagged by DISC. Figure 3.7 shows the distribution of a typical positive beam data where all three beam particle types are well separated by the successful use of the combined TRD and DISC data.

The E769 TRD consisted of 24 identical radiator-chamber modules, each made of a stack of $20012.7 \mu \mathrm{m}$ thick polypropylene foils separated by $180 \mu \mathrm{m}$ thick spacers. Figure 3.8 shows one of these modules where the TR was detected by two succesive 


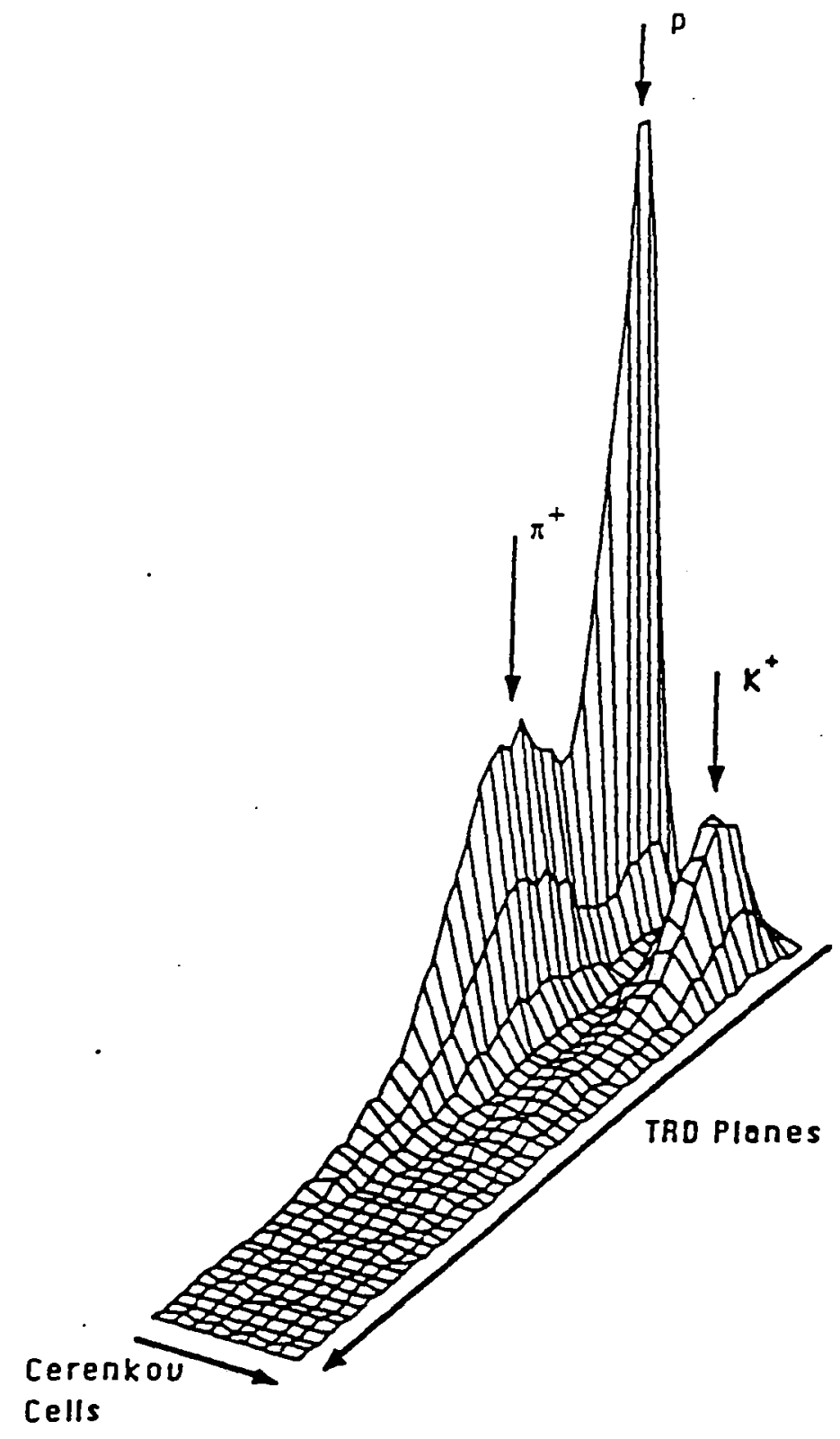

Figure 3.7: DISC PMT coincidences vs TRD plane counts. 


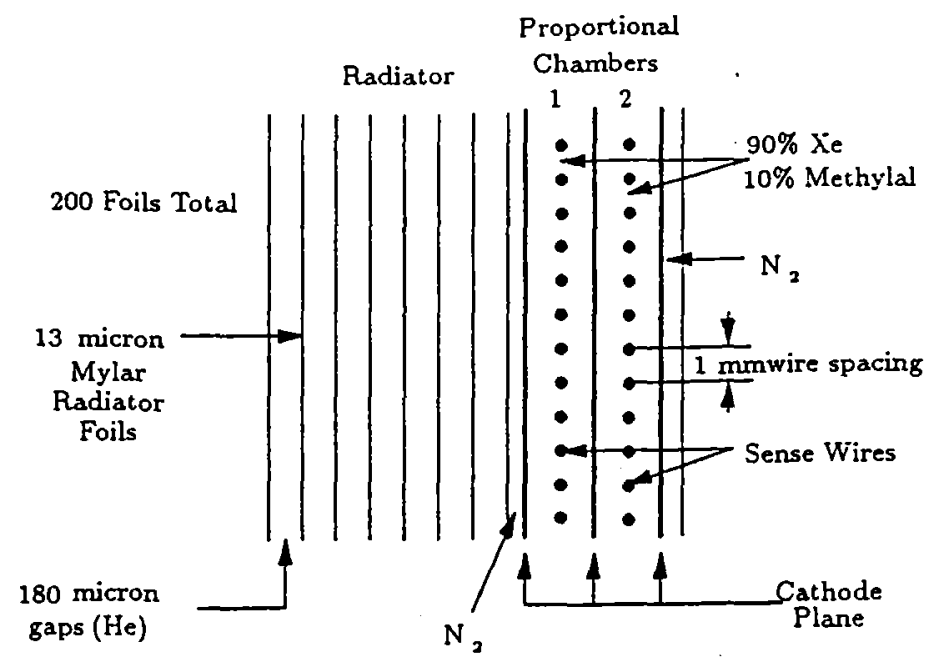

Figure 3.8: Schematic of a TRD radiator-chamber module.

multi-wire (64 wires per plane) proportional chambers designed at Fermilab (See the following section on MWPC for more details.). The chambers were filled with a gas mixture of $90 \%$ xenon and $10 \%$ methylal which proved to be stable over a wide range of operating voltages with a large linear response to incident photons. The radiator gaps were flushed with helium since it provides a better medium for transition radiation than air. Nitrogen was sent through a buffer region between the radiator and the chamber to keep $\mathrm{He}$ from contaminating the $\mathrm{Xe}$ mixture.

The 64 wires in each proportional chamber were connected in sets of four, and the 16 output signals were sent to the input of the amplifier/discriminator cards. The outputs of the discriminators were connected to the inputs of LeCroy 4564 , 16 channel OR gates modules. The output signals of these modules were counted as plane hits which were read out by CAMAC through LeCroy 4448 latch modules. Figure 3.9 shows the plane count distribution for typical positive beam particles which were not tagged as kaons by the DISC. Similar histograms were made for each run, and fitted with two binomial distributions. From these histograms, the efficiency for the selection of one particle type and its contamination by another were computed. Table 3.4 contains the results of the fits to these histograms for the efficiency and 


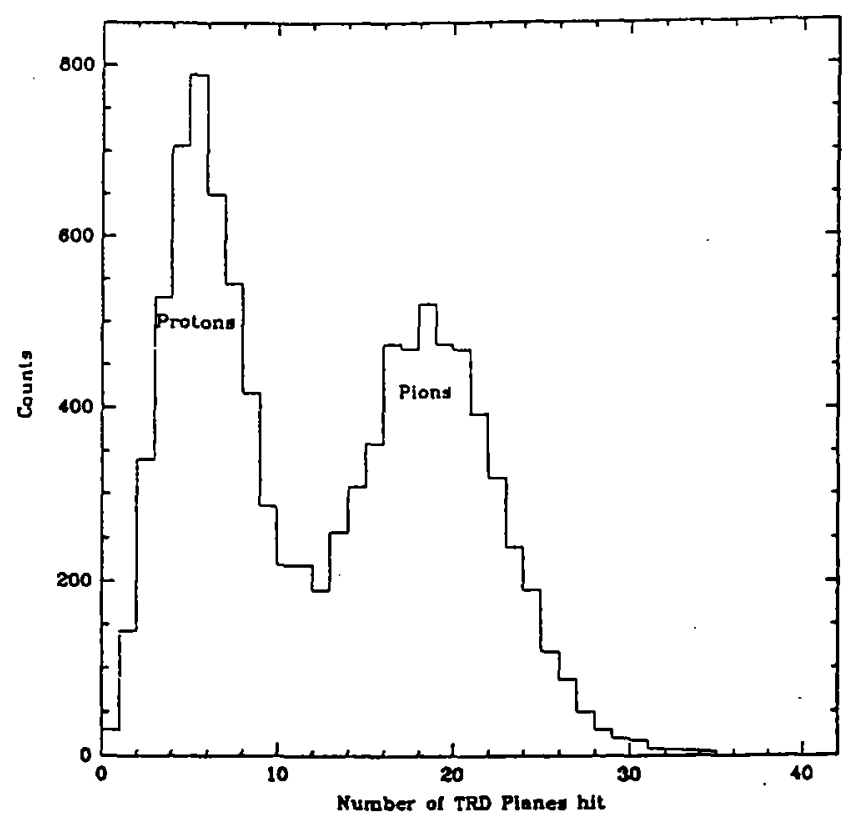

Figure 3.9: TRD plane count distribution

contamination of pions and protons as a function of a cut on the plane counts.

\subsubsection{Multiwire Proportional Chambers (MWPC)}

A proportional wire chamber consists essentially of a gas container subjected to an electric field between a plane of anode wires and two cathode planes on the sides. An energetic charged particle passing through the chamber ionizes the gas, and the

\begin{tabular}{|c||c|c|c|}
\hline \multicolumn{2}{|c|}{$\begin{array}{c}\text { No. of TRD planes } \\
\text { that fired }\end{array}$} & efficiency & contamination \\
\hline \hline \multirow{4}{*}{ Protons } & $<6$ & $87.5 \%$ & $1.8 \%$ \\
& $<7$ & $92.9 \%$ & $3.1 \%$ \\
& $<8$ & $95.7 \%$ & $5.3 \%$ \\
\hline \multirow{4}{*}{ Pions } & $\geq 8$ & $94.8 \%$ & $4.1 \%$ \\
& $\geq 10$ & $86.8 \%$ & $2.2 \%$ \\
& $\geq 12$ & $73.0 \%$ & $1.7 \%$ \\
\hline
\end{tabular}

Table 3.4: TRD efficiencies and contaminations 
produced electrons and ions are collected at the electrodes providing an electric signal indicating the passage of the particle through the chamber. The chamber is called proportional when the electric field is large enough for the electrons to cause secondary ionizations, but small enough so that the output signal is proportional to the number of primary ions.

Some of the properties of a suitable gas for proportional chambers are: low working voltage, good proportionality, fast recovery, high rate capability, and long lifetime [24]. To provide as many of these characteristics as possible, one usually uses a mixture of gases. We used a mixture of $82.7 \% \mathrm{Ar}, 17 \% \mathrm{CO}_{2}$, and $0.3 \%$ Freon$13 \mathrm{~B} 1$. Argon is used as the main component since as a rare gas it has a large multiplication at relatively low voltages but it is more economical as compared to $X e$ or $K r$. However, the photons emitted due to the de-excitation of excited argon atoms can cause secondary emissions at the cathode. To avoid this, it is a common practice to mix argon with a polyatomic gas such as $\mathrm{CO}_{2}$. The polyatomic gases absorb photons due to their many rotational and vibrational levels, and they deexcite through elastic collisions or dissociation into simpler molecules. For still higher gains, we add a small amount of an electronegative gas such as freon, which in addition to its photon-quenching capability, can capture the free electrons which can induce avalanches.

We used two types of MWPCs in the experiment: the smaller beam chambers, and the large spectrometer MWPCs. The beam chambers were designed and built at Fermilab Experimental Areas Department for use in the secondary beam lines [25]. They were placed upstream of the target in two stations with co-ordinates -3152 and $-1223 \mathrm{~cm}$ along the beamline [26]. Each station consisted of four wire anode planes and five cathode planes. Even though there were four planes of $\mathrm{X}, \mathrm{X}^{\prime}, \mathrm{Y}$, and $\mathrm{W}$ views, there were basically only two types of wire plane that were used in building these chambers: the "straight" plane and the "slant" plane. The straight plane has 128 wires with $1-\mathrm{mm}$ spacings between them. The wires on this board are positioned such that the 64th wire is $0.25 \mathrm{~mm}$ off of the board center line. The purpose of such design was to achieve a $1 / 2 \mathrm{~mm}$ effective wire spacing with a combination of two similar wire planes in opposite directions. The $\mathrm{X}, \mathrm{X}^{\prime}$, and $\mathrm{Y}$ views were straight planes 
of which only the middle 64 wires were used. The $\mathrm{W}$ plane, on the other hand, was a slant plane of 64 wires with $1-\mathrm{mm}$ spacings which measured the coordinates rotated by 60 degree relative to our $X$ axis. The wires on the sense planes are 0.0004 -inch gold plated tungsten.

The cathode planes are 0.0005-inch aluminum foils. The gap between a sense plane and a cathode plane is 0.125 inch. High voltage $(-2750 \mathrm{~V})$ was supplied to the cathode planes through $100 \mathrm{~K} \Omega$ current limiting resistors on the planes. The high voltage power supplies were equipped with circuitry to shut off the voltage and discharge the chamber when a sudden surge of current was detected.

The spectrometer MWPCs consisted of two Y-view chambers positioned 1.5 meter downstream of the target along with the first set of drift chambers just before the first magnet. Each chamber consisted of a single wire plane of 288 wires with $2-\mathrm{mm}$ spacings between two cathode planes of thin aluminized mylar sheets held at -3500 volts. The active area for each chamber was $57.6 \times 57.6 \mathrm{~cm}^{2}$.

To process the output signals of our chambers, we used PCOS III, a compelete Proportional Chamber Operating System developed by LeCroy. This system consisted of 16-channel amplifier/discriminator cards (Model 2735PC) ${ }^{1}, 32$-channel delay and latch modules (Model 2731A), crate readout controller (Model 2738), and buffer/CAMAC interface (Model 4299).

In the case of the beam chambers, the amplifier cards were mounted on the sense planes ( 4 cards/plane). However, for the spectrometer chambers, due to the large number of cards required per plane (18), and for the sake of accessibility and convenience in maintenance, the cards were housed in special crates (18 cards/crate), and their inputs connected to the anode outputs of the chambers through ribbon cables. The outputs of the cards were in turn connected to the inputs of the Model $2731 \mathrm{~A}$ receiver modules through twisted-pair ribbon cables.

In order to account for the decision time of the trigger logic, and the difference in the arrival time of the signals from different chambers, the latch modules contain a programmable delay for each channel. The delay of all channels of each module may be commonly set over the range of $300-628.5 \mathrm{nsec}$ in $1.5 \mathrm{nsec}$ steps. The hit-wire data

\footnotetext{
${ }^{1}$ In place of these cards, we used similar cards designed by Nanometric Systems.
} 
contained within the 2731A modules are stored as 32-bit words. Each 2731A module provides a LAM (Look At Me) signal to allow only those modules containing hits to be read.

The 2738 controller in each crate rapidly scans the LAM status of $2731 \mathrm{~A}$ modules and encodes the address of the hit wires only in the modules with hits. The data are loaded into a memory for subsequent CAMAC readout. The coincidence gate received by the 2738 module is transmitted to the $2731 \mathrm{~A}$ latch modules via the Dataway (on the back of the CAMAC crate). The trailing edge of the gate activates the readout sequence. The controller scans the LAM status of all modules in $100 \mathrm{nsec}$, and performs the readout of the modules with hits at a rate of $100 \mathrm{nsec}$ per module. The controller is double-buffered allowing a second module to be read immediately while the data from the first are being processed by a fast encoder. The addresses of the hit wires are generated at a rate of $10 \mathrm{M}$ words/sec. The 2738 module contains a look-up memory to allow the user to assign logical addresses to the latch modules. Wire addresses of a system using one crate for more than one wire plane can be assigned to eliminate the possibility of confusing the hits from different planes. The encoded 14-bit addresses of hit wires contain the logical module address and the wire sub-address.

Communication with the CAMAC crates housing the PCOS III was made via

the LeCroy Databus. It allowed the compact formatted data to be automatically transferred from the memory of the 2738 controller to the 4299 databus interface. Data were also written through the Model 4299 into the 2738 module, such as the logical addresses and delay settings of the 2731A modules. The 4299 module can be reset or cleared with NIM pulses applied to the front-panel Lemo-type connectors. Figure 3.10 shows the basic configuration of the PWC readout system.

\subsubsection{Silicon Microstrip Detectors (SMD)}

Semiconductor detectors behave much like ionization chambers. Incident charged particles deposit ionization energy and dislodge electrons, which in turn produce secondary ionizations. The electron-hole pairs generated as the result of the ionization drift apart toward the electrodes due to the electric field of the bias voltage to form 


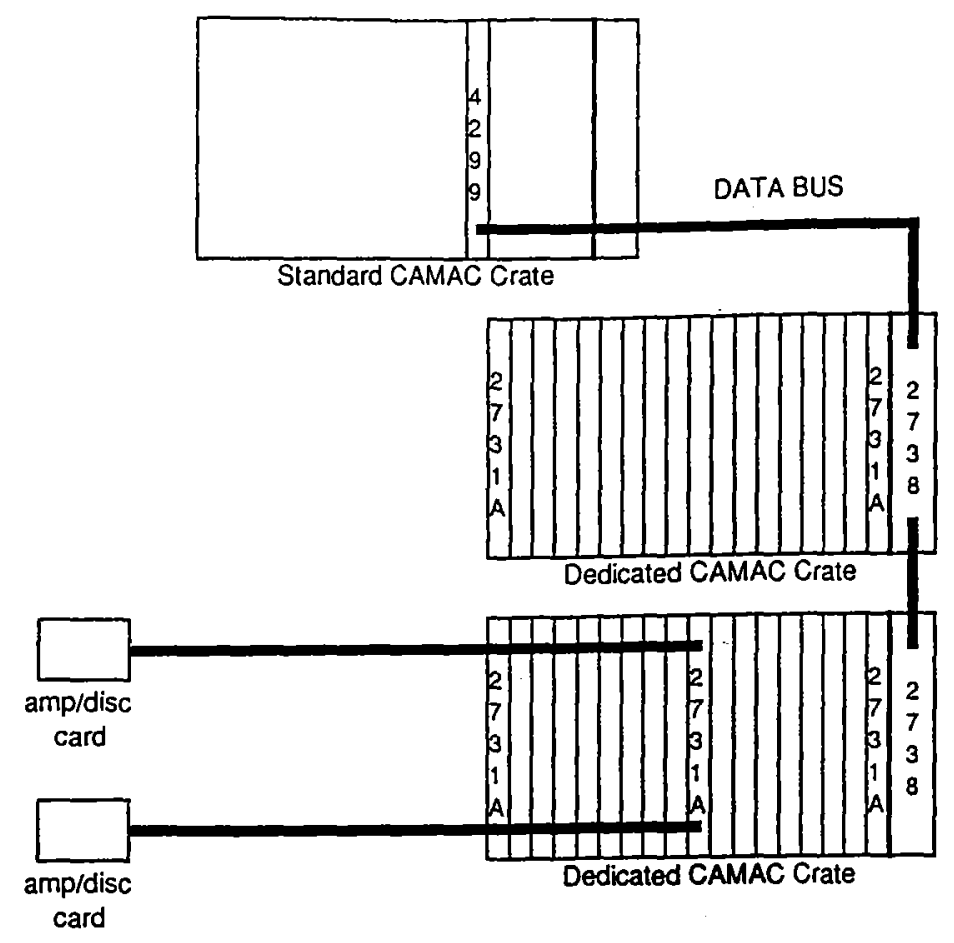

Figure 3.10: PWC readout configuration in E769.

signals proportional to the deposited energy.

There are advantages to the use of semiconductor detectors instead of gaseous ionization chambers. It takes only $3.6 \mathrm{eV}$ to produce an ion pair in silicon compared to $\sim 30 \mathrm{eV}$ in argon. The high density of silicon is another factor in the large number of the ion pairs produced. A minimum ionizing particle passing through a $300 \mu \mathrm{m}$ silicon detector typically produces 25000 electron-hole pairs, compared to about only 3 ion pairs in the same thickness of the standard drift chamber gas. Silicon can also be etched to give narrow strips of active detector. This provides a device which behaves like a MWPC but with a better spatial resolution. Therefore, semiconductor devices are useful when a thin, high resolution detector is required near an interaction region. The disadvantages, on the other hand, are the small active area and the radiation damage. Figure 3.11 shows the cross section of an SMD plane.

E769 used a total of 13 silicon microstrip planes positioned in 5 stations. Station A, upstream of the target, consisted of two planes (AX, AY) used in conjunction with the upstream PWC planes to track the beam. The other stations were placed downstream of the target to find the interaction and decay vertices. Station $B$, like station A, consisted of two planes. Each plane had 384 strips of $25 \mu \mathrm{m}$ pitch in the 


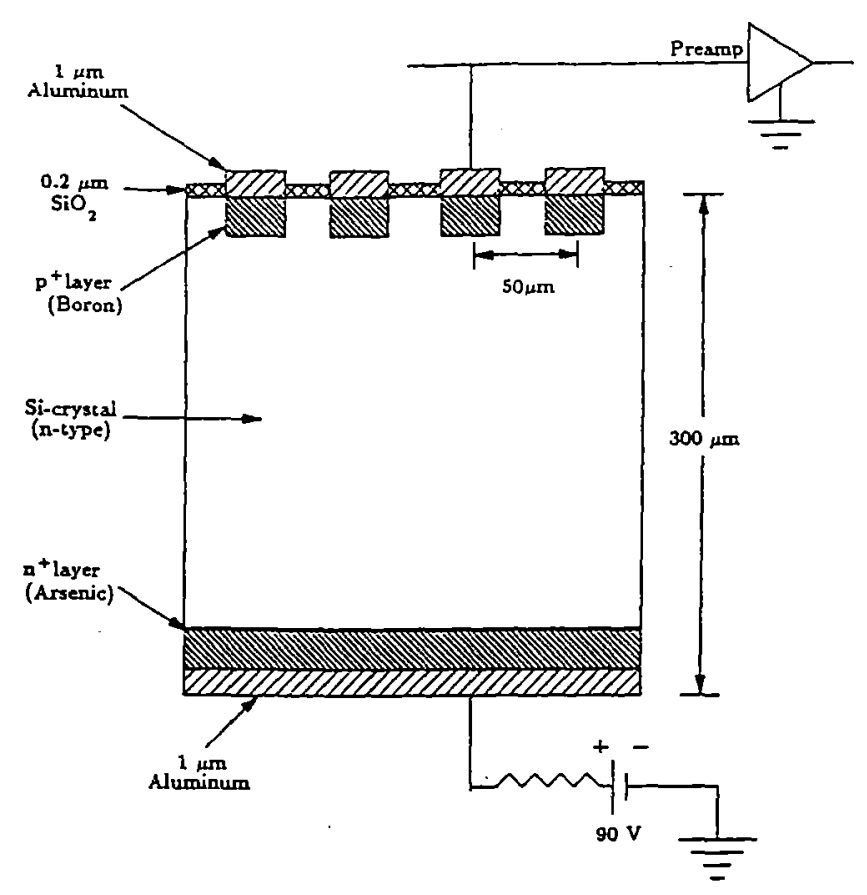

Figure 3.11: Cross Section of an SMD Plane

\begin{tabular}{|c||c|c|c|c|c|}
\hline SMD assembly & $\mathrm{A}$ & $\mathrm{B}$ & 1 & 2 & 3 \\
\hline Coordinates & $\mathrm{X}, \mathrm{Y}$ & $\mathrm{Y}, \mathrm{X}$ & $\mathrm{X}, \mathrm{Y}, \mathrm{V}$ & $\mathrm{Y}, \mathrm{X}, \mathrm{V}$ & $\mathrm{X}, \mathrm{Y}, \mathrm{V}$ \\
\hline Pitch $(\mu \mathrm{m})$ & $25 / 50$ & $25 / 50$ & 50 & 50 & 50 \\
\hline $\begin{array}{c}\text { Instrumented } \\
\text { strips }\end{array}$ & 384 & 688 & 512 & 768 & 1000 \\
\hline Avg. efficiency & $74 \%$ & $72 \%$ & $94 \%$ & $95 \%$ & $90 \%$ \\
\hline
\end{tabular}

Table 3.5: E769 SMD characteristics.

center, and 152 strips of $50 \mu \mathrm{m}$ pitch on the sides [27]. The next three stations 1 , 2 , and 3 consisted of three planes (X, Y, V) each. The $\mathrm{V}$ view planes were tilted by $20.5^{\circ}$ with respect to the $\mathrm{X}$ planes. These planes had strips of $50 \mu \mathrm{m}$ pitch. Table 3.5 summarizes some of the characteristics of the E769 SMD planes.

As shown in figure 3.11 , the charge collected at the aluminum strips was preamplified. Then the signal was sent on shielded ribbon cables to the amplifier/discriminator/latch cards. These cards were read out serially by Nanoscanner CAMAC modules. 


\begin{tabular}{|c||c|c|c|c|}
\hline DC Station & 1 & 2 & 3 & 4 \\
\hline \hline No. of planes & 8 & 12 & 12 & 3 \\
Dimensions $(\mathrm{cm} \times \mathrm{cm})$ & $160 \times 120$ & $230 \times 200$ & $330 \times 200$ & $550 \times 300$ \\
Cell size $(\mathrm{cm})$ & 0.476 & $\mathbf{0 . 9 5 3}$ & 1.588 & 3.175 \\
\hline
\end{tabular}

Table 3.6: E769 DC system characteristics.

\subsubsection{Drift Chamber (DC)}

A drift chamber is another tracking device. It uses the drift time of the ionization electrons to measure the position of the ionizing particle. Drift chambers are usually used for covering large solid angles, with the same resolution as MWPCs' but with fewer wires and a lower cost.

E769 used a total of 35 drift chambers, divided into four stations (D1, D2, D3, and D4). Refer to figure 3.4 for the positions of these stations. Each drift chamber assembly consisted of sense wire planes sandwiched between cathode planes. Successive sense wire planes in the same assembly shared the cathode plane between them. Each sense wire plane was made up of alternating sense wires and field shaping wires. The cathode planes were planes of wires held at a negative high voltage (typically $-2.4 \mathrm{kV}$ ) relative to the sense wires, while the field shaping wires were at a slightly less negative voltage (about $-2.0 \mathrm{kV}$ ).

Each chamber assembly contained three or four planes. D1 contained two assemblies of planes which measured $\mathrm{X}, \mathrm{X}^{\prime}, \mathrm{U}$, and $\mathrm{V}$ coordinates (where $\mathrm{X}^{\prime}$ is offset from $\mathrm{X}$ by $1 / 2$ the wire spacing, and $U$ and $V$ wires make $\pm 20.5^{\circ}$ angle with the vertical. See figure 3.12). D2 and D3 contained four assemblies of $\mathrm{X}, \mathrm{U}$, and $\mathrm{V}$ planes each, while D4 contained only one triplet of $\mathrm{X}, \mathrm{U}, \mathrm{V}$ planes. Table 3.6 summarizes the physical characteristics of E769 DC system.

The gas used in the drift chambers was a mixture of equal proportions of argon and ethane with about $1 \%$ ethanol used as both a quencher and an anti-aging agent. The ethanol was added by bubbling the 50/50 argon-ethane mixture through $0^{\circ} \mathrm{C}$ ethanol. The ratio of the gas mixture (in this case, 50/50) is important in achieving drift velocities independent of the electric field. This minimizes the change in drift 


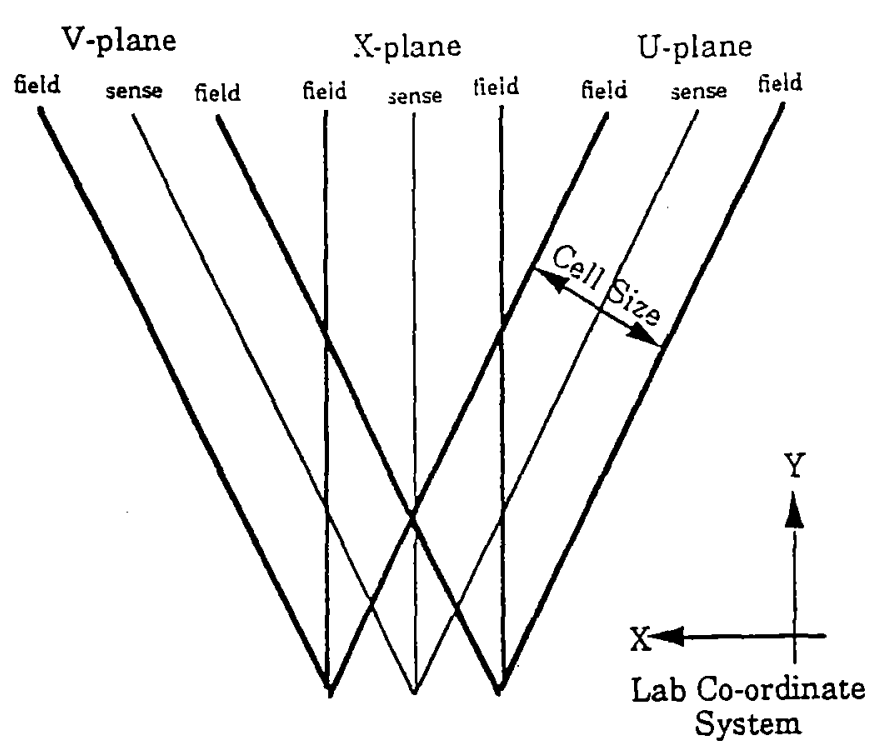

Figure 3.12: Orientation of $\mathrm{X}, \mathrm{U}$, and V Planes

velocity as the electrons approach the sense wires, and consequently the linear spacetime correlation, which is essential in our measurements, can be maintained.

E769 DC system contained 6288 sense wires. The output signals from these wires were amplified and discriminated at the chamber by LeCroy DC201 or Nanomaker N-277C amplifier/discriminator chips [18]. The ECL (Emitter Coupled Logic) output signals of the discrimintors were then carried by twisted pair ribbon cables to the STARTs of the LeCroy 4291 TDCs (time to digital convertor). Each CAMAC crate housing the TDCs was controlled by a LeCroy 4298 module. It received the experimental STOP signal, and distributed it to all the TDC modules in its crate, and then instructed them to digitize their data. The digitized times were then read by the 4298 module and sent to a dedicated, Femilab built, P4299 module which re-formatted the data and raised a LAM to signal its readiness to be read out by the Smart Crate Controller in the DA system. 


\begin{tabular}{|c||c|c|}
\hline Magnet & M1 & M2 \\
\hline \hline Entrance Aperture $(\mathrm{cm} \times \mathrm{cm})$ & $154 \times 73$ & $154 \times 69$ \\
Exit Aperture $(\mathrm{cm} \times \mathrm{cm})$ & $183 \times 91$ & $183 \times 86$ \\
Length $(\mathrm{cm})$ & 165 & 208 \\
Current $(\mathrm{A})$ & 2500 & 1800 \\
$\int B_{y}(0,0, z) d z(\mathrm{~T}-\mathrm{m})$ & 0.71 & 1.07 \\
$p_{t}$ kick $(\mathrm{MeV} / \mathrm{c})$ & 212.4 & 320.7 \\
\hline
\end{tabular}

Table 3.7: E769 magnets characteristics.

\subsubsection{Analysis Magnets}

E769 used two large aperture magnets (M1 and M2) to measure the track momenta. Figure 3.4 shows the positions of these magnets in the spectrometer. The fields in both magnets were in the $-y$ direction. From the equation for the force on a particle of charge $q$ moving in a magnetic field of $B$, we obtain:

$$
d \vec{p}=q d \vec{l} \times \vec{B}
$$

Using this equation for a particle moving along the $z$-axis, one can calculate the transverse momentum $\left(p_{t}\right)$ kick on the particle by the magnets to be:

$$
p_{t}=q \int B_{y} d z
$$

The angle by which the trajectory of the particle is deflected is given by $\theta=p_{t} / p$. Combining this relation with equation 3.8 gives the formula for $\theta$ in terms of the momentum:

$$
\theta=\frac{q \int B_{y} d z}{p}
$$

The charactristics of the two magnets M1 and M2 are summarized in table 3.7.

\subsubsection{Electromagnetic (EM) Calorimeter}

E769 used a lead-liquid scintillator EM shower detector, designated as SLIC (Segmented Liquid Ionization Calorimeter) in figure 3.4, to measure the energy of the 
particles which interacted predominantly through electromagnetic interactions. The SLIC has an active area of $244 \mathrm{~cm}$ by $488 \mathrm{~cm}$ and is segmented into $3.17 \mathrm{~cm}$ wide strips. Along the beam direction, it consists of 60 layers of $0.63 \mathrm{~cm}$ thick lead and $1.27 \mathrm{~cm}$ thick liquid scintillator.

The layers are arranged alternately in three directions of $U, V$, and $Y$ to determine the position of the shower. Figure 3.13 shows the cross sectional view of the internal structure of the SLIC. The most upstream section consists of a steel "Wirecomb panel" which also appears at the back of the SLIC to provide structural integrity to the whole detector while at the same time produce the least amount of material along the incident particle's path.

The radiator sheets are teflon-coated aluminum-lead-aluminum laminates which are placed between the scintillation layers, as shown in figure 3.13 . The scintillation layers, in turn, are formed by thin corrugated aluminum sheets, coated with teflon, and filled with a scintillating liquid with an index of refraction greater than that of teflon. As a result, the light generated in the scintillator propagates along the teflon channel within the total internal reflection cone. One end of each channel is terminated by a $90^{\circ}$ mirror of $\sim 80 \%$ reflectivity. The reflected light is transmitted by a wavelength shifter bar onto a phototube. The wavebar is a lucite-type material doped with the chemical BBQ which shifts the wavelength of the scintillating light from blue to green where the phototube is more sensitive. A schematic view of the SLIC is shown in figure 3.14 where the cutaway reveals the first layer of the $U, V$, and $\mathrm{Y}$ views.

Each of the SLIC phototubes was assigned to one channel of a 12-bit LeCroy $2285 \mathrm{~A}$ ADC. The fractional energy resolution for SLIC was found to be $\Delta E / E \simeq 21 \% / \sqrt{E}$, where $E$ is measured in $\mathrm{GeV}$. For more details on the SLIC, refer to [28].

\subsubsection{Hadronic Calorimeter}

The hadronic calorimeter at TPL is an iron-acrylic scintillator device which, in conjunction with the EM calorimeter (SLIC), was used in triggering on the hadronic events. The hadrometer is made up of two identical modules as shown in figure 3.14. Each module has 18 layers of $2.5 \mathrm{~cm}$ thick steel plate alternating with $0.95 \mathrm{~cm}$ thick 


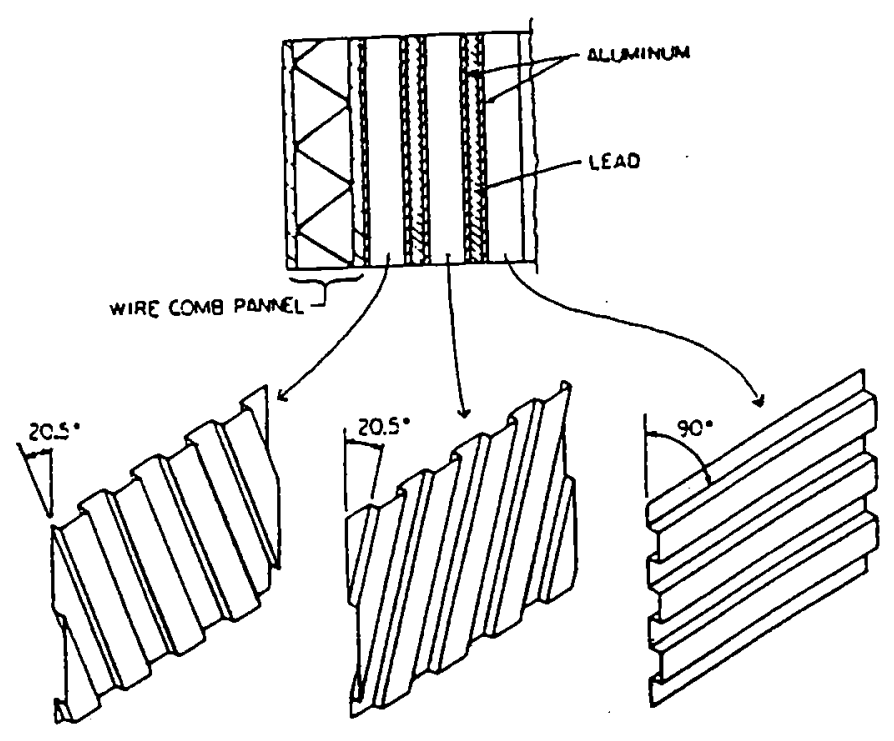

Figure 3.13: SLIC radiator-scintillator assembly.

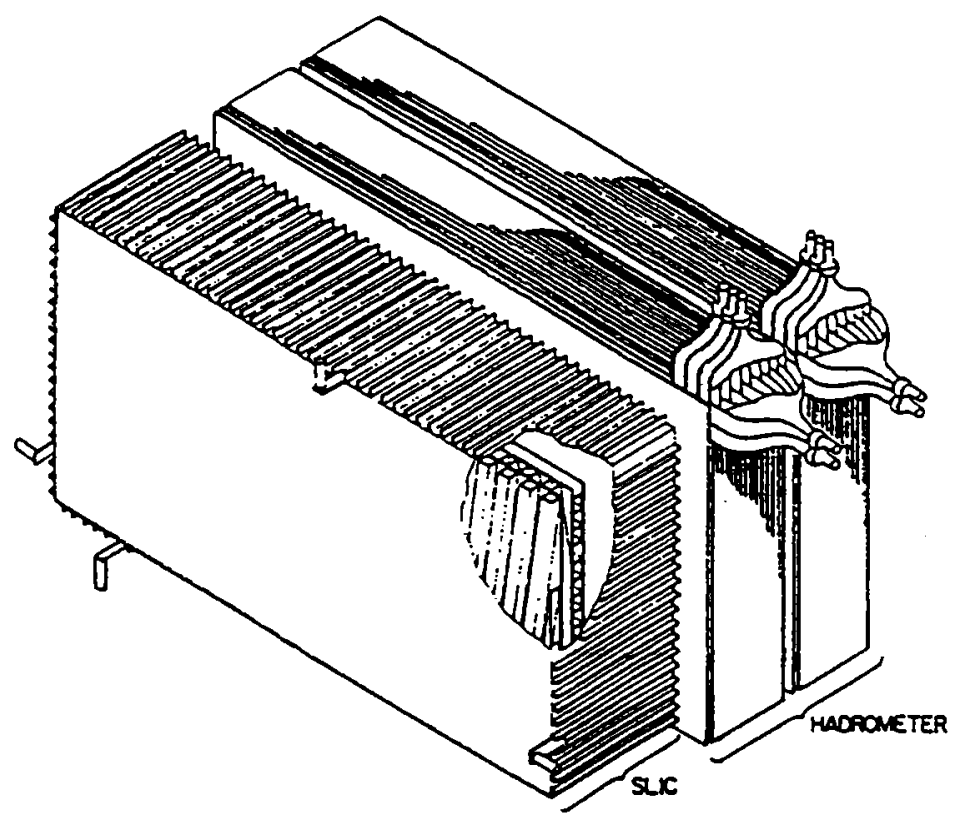

Figure 3.14: Schematic view of E769 calorimeter system. 
layers of plastic scintillators. Each steel plate is $490 \mathrm{~cm}$ wide and $270 \mathrm{~cm}$ high, while the scintillating layers are made up of $14.5 \mathrm{~cm}$ wide strips arranged in $\mathrm{X}$, and $\mathrm{Y}$ directions alternately.

This detector is of classic design where scintillators are attached to light guides which channel the light to PMTs. The anode signal from each PMT is integrated in a charge sensitive ADC (LeCroy 2285A). The energy resolution of the hadrometer was found to be $\Delta E / E=75 \% / \sqrt{E}$, with $E$ in GeV. For a more detailed description of the hadrometer, see [29].

\subsection{Trigger}

E769 collected approximately 370 million events using two basic types of physics triggers: minimum-bias interaction $(I N T)$ and large transverse energy $\left(E_{t}\right)$ triggers. About $6 \%$ of the data were taken with $I N T$ trigger and the rest with variations of $E_{t}$ trigger. The selection of $E_{t}$ trigger was based on the observation that the mean transverse momentum of a particle produced in an inlastic hadronic interaction is on the order of its mass. Since charm hadrons have larger mass relative to lighter hadrons, we could enrich our charm sample by selecting a high $E_{t}$ threshold. INT trigger on the other hand provided us with a min-bias sample which could be used for comparable non-charm production studies. The details of both triggers are described below.

- INT - This trigger made use of three scintillation paddles: beam spot, beam halo, and interaction (Fig. 3.3). The thresholds in the discriminators for the beam spot and the beam halo were set to that for a single minimum ionizing (MI) particle while the threshold for the interaction counter was set for at least $3 \mathrm{MI}$ particles. The logic of the trigger, indicated below, required the anti-coincidence of the beam halo and beam spot, i.e. the beam go through the beam spot counter and the hole in the halo (veto) counter, in coincidence with the interaction signal.

$$
I N T=\operatorname{spot} \cdot \overline{\text { halo }} \cdot \text { interaction }
$$

Condsidering the relatively high beam rate $(\sim 1 \mathrm{MHz})$ and low data aquisition 
(DA) rate $(450 \mathrm{~Hz})$, and taking into account the interaction length of the target ( $\sim 2 \%$ ), we prescaled $I N T$ trigger by factors of $100-500$ in order to avoid writing too many interaction events on tape, but enough to collect a sizable sample of minimum bias data.

- $E_{t}$ - The transverse energy of each event was measured approximately using the calorimeters. The signal from each calorimeter module was weighted proportionally to its distance from the center of the calorimeter. The summed signal was then sent to the inputs of two separate discriminators with thresholds corresponding to transverse energies of approximately $5.5 \mathrm{GeV}$ ( $E_{t}$ threshold) and $9.0 \mathrm{GeV}$ ( $E_{t B}$ theshold). The logic for this trigger required the coincidence of the above discriminators output signals with the INT trigger.

For the positive beam runs, an additional requirement was included in the $E_{t}$ trigger logic. It required the veto of any beam particle within $150 \mathrm{~ns}$ of another beam particle. This was to ensure the proper functioning of the transition radiation detector (TRD) in distinguishing between the pions and the protons. This was called "beam killer." A schematic of the trigger is shown in figure 3.15.

\subsection{Data Acquisition System}

The general function of a DA system is to write the information gathered through front-end digitizers in a compressed format onto a storage medium such as a magnetic tape. The two most important characteristics of a DA system are the throughput and the dead time. Throughput is the speed at which data can be taken, and dead time is the fraction of time the system is unable to receive any data because it is busy processing the data already in its buffers.

E769 DA system was able to collect data at a rate of $1.8 \mathrm{Mb} / \mathrm{s}$ (450 events $/ \mathrm{s}$ ) with $25 \%$ dead time $[26,30]$. This was achieved by employing a multiprocessor based parallel architecture DA system shown schematically in figure 3.16. A brief description of the hardware configuration and on-line monitoring of the DA system follows. 


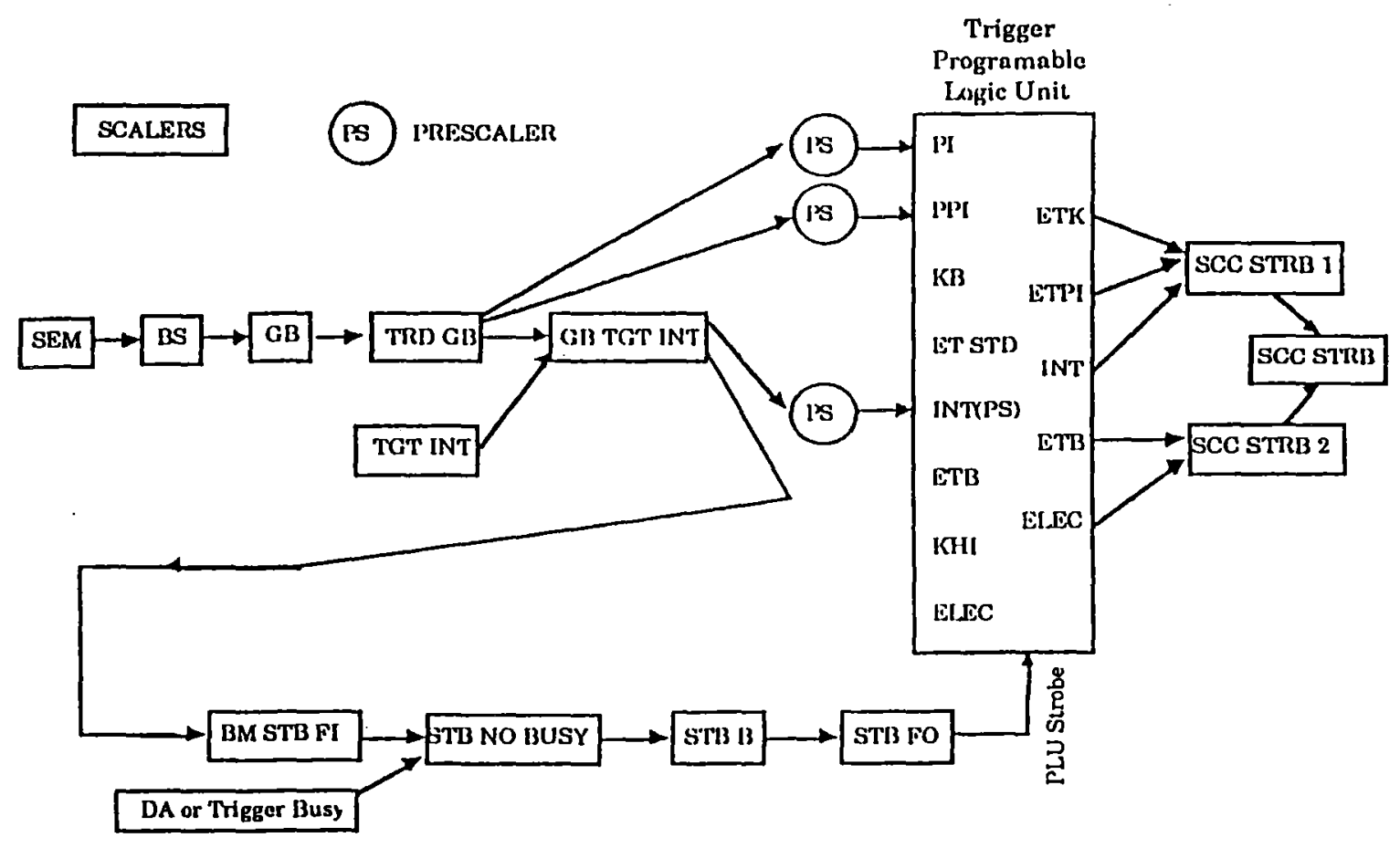

Figure 3.15: Diagram of E769 trigger. 


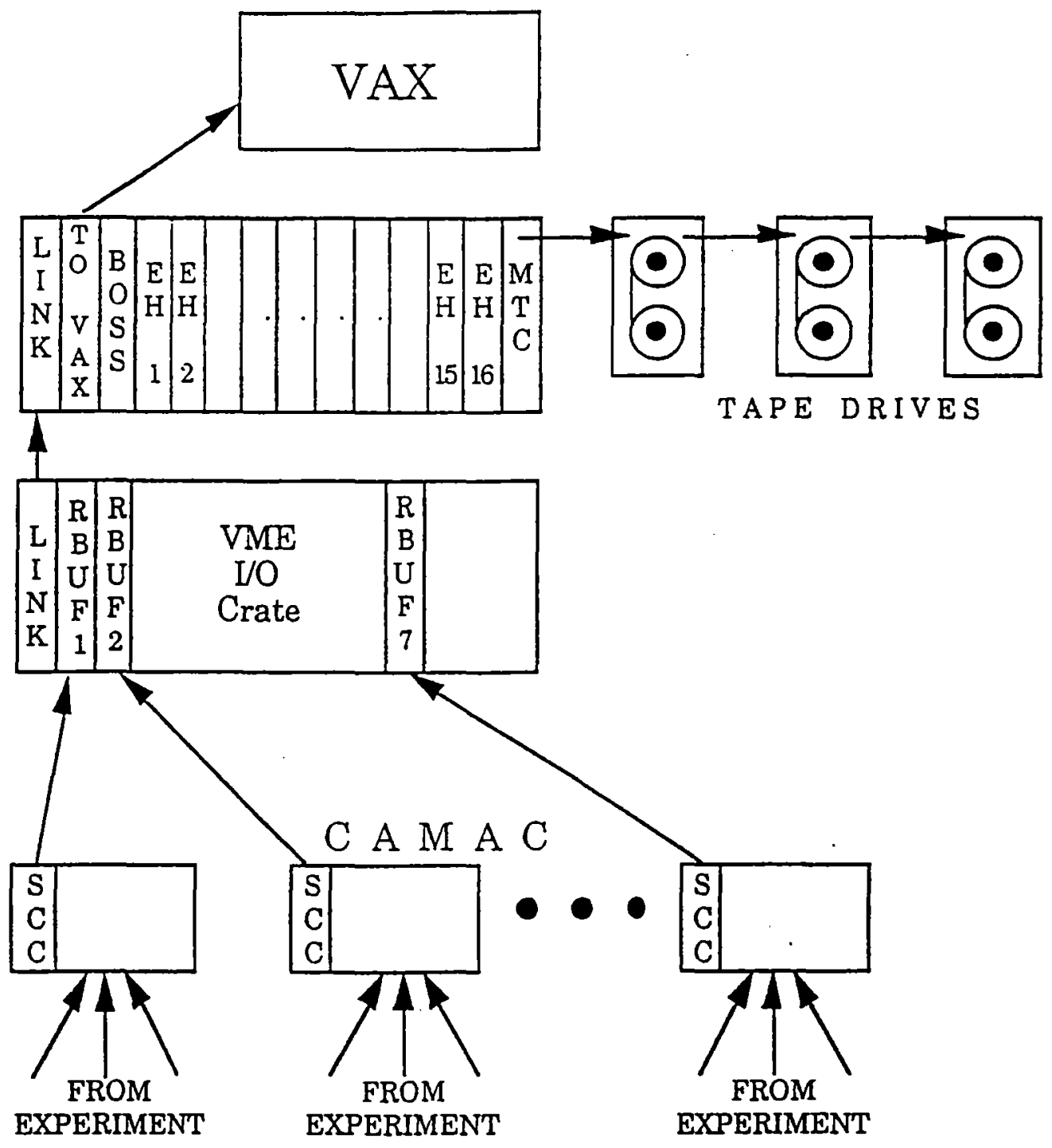

Figure 3.16: Schematic of the E769 data acquisition system. 


\subsubsection{Hardware Configuration}

As shown in figure 3.16, the signals from the detectors were digitized by CAMAC modules housed in seven CAMAC crates. Each crate was furnished with an auxiliary controller named Smart Crate Controller (SCC) with a microprocessor containing the instructions on how to read the modules in the crate. At the start of every run, these readout lists were downloaded by a VAX 11/780 through a connection to RS-232 port on each SCC.

The SCC on each CAMAC crate sent the digitized events through its data port to a VMEbus module called Readout Buffer (RBUF) to be stored. The readout of all CAMAC crates was done in parallel to reduce the readout time. The RBUFs were dedicated, double-buffered memory modules with $32 \mathrm{~K}$ block of memory per buffer. These two blocks of memory allowed a new event to flow into one buffer while the old event was still being read out from the other buffer.

The data in RBUFs were read out by microprocessors developed by the Advanced Computer Project (ACP) group at Fermilab called ACPs. There were 17 of these modules in the principal VME crate which was linked by a Bus-extender module to the VME I/O crate containing the RBUFs. Of the $17 \mathrm{ACPs}$, one was assigned as Boss and the rest as Event Handlers (EH). The Boss was responsible for managing the operation of the Event Handlers. At any given time, two of the Event Handlers were designated as Grabbers, and the other 14 as Munchers. The Grabber's job was to build the events from the event fragments coming from the RBUFs and to place them in input buffers while the Munchers compressed and formatted the previously grabbed events and put them in output buffers.

Other than the ACP modules, the main crate contained a VME Resource Module (VRM) handling the bus arbitration, a Branch Bus controller and a Branch Bus-VME interface linking the VME crate to the VAX 11/780 via a DR11-W. The crate also housed a Ciprico magnetic tape controller (MTC) which handled the tape writing. The VAX ran the System Controller program which served as the operator interface to the DA. Through this program, one had the ultimate control of the system to issue commands to start or to end a run, or to monitor the performance of the detectors by interfacing with the on-line event pool. 


\subsubsection{On-Line Monitoring}

E769 used various methods to monitor the progress of the experiment and the status of its detectors. Some of the more important of these methods were the Detached Analysis Process (DAP) system, the Interspill process control, and the E769 Event Display.

- DAP - DAPs were monitoring programs which used the events stored in the event pool to display updates of the status or the performance of the detectors in the form of tables, histograms and error messages.

o Interspill - This process was run between the spills ( $<38$ seconds) to avoid interference with the DA's control of the CAMAC. It was used for calibration and monitoring purposes which required direct access to the CAMAC system, such as reading the pedestal levels of the ADC modules.

o Event Display - This graphics package enabled the experimenter to directly observe the performance of the detectors by tracking the particle paths through the detectors, displaying the hits in the Čerenkov phototubes and the energy distributions in the calorimeters, etc. 


\section{Chapter 4}

\section{Analysis}

This chapter describes the different levels of analysis from the initial stage of the conversion of the raw data into physical events to the final process of charm signal extraction. In the first section, we explain the long process of data reconstruction which lasted over a year using a large amount of computing. In the second section, we give a brief discussion of our Monte Carlo which helped us simulate our experiment in a realistic fashion and project actual physics results. And finally in the third section, we describe our selection criteria for the extraction of our $D^{0} \rightarrow K^{-} \pi^{+} \pi^{-} \pi^{+}$signal.

\subsection{Data Reconstruction}

The conversion of the raw digital information (from the digitizing modules recorded by the DA) on magnetic tapes into meaningful physical properties (of the produced particles) such as trajectories, momentum, energy, and particle type probability, is called reconstruction. In the case of E769, this conversion was achieved through a reconstruction process consisting of the following subprocesses: PASS0, PASS1, PASS2.

\subsubsection{PASS0}

Before starting the actual reconstruction, the first 1000 events on each tape went through a preliminary pass which extracted such information as the ADC pedestal 
values, TRD plane counts, and SMD hot channels. Later on, during the reconstruction, PASS0 code was called to collect such statistics as the average number of DC hits per plane, DISC PMT and TRD plane count frequencies, and trigger latch bits. At the conclusion of the reconstructon process, a summary file was prepared containing such information as: average number of tracks, average number of vertices, etc. which later proved invaluable in pointing out regions of data which were suspect due to the detector's malfunctioning.

\subsubsection{PASS1}

The actual reconstruction started with pattern recognition; i.e., tracking the trajectories of the particles in each event. First, the SMD hits were used to reconstruct the tracks originating upstream of the magnets. The hits in downstream MWPCs and D1 were also used to complement the SMD planes. We fitted all the SMD hits to a straight line by minimizing $\chi^{2}$ expressed as:

$$
\chi^{2}=\sum_{i=1}^{n} \frac{\left(x_{i}^{p}-x_{i}^{m}\right)^{2}}{\sigma_{i}^{2}}
$$

where $x_{i}^{p}$ is the predicted position of the fitted track at the $i$ th plane, $x_{i}^{m}$ is the position of the hit found in the same plane, and $\sigma_{i}$ is the error on the measurement of $x_{i}^{m}$.

Next, these tracks were extrapolated through the magnets and D2 to be linked to hits found downstream of the magnets in D3 and D4. This process provided the track parameters and momenta for the detected charged particles. After this stage, a second search was done to find the tracks originating downstream of the SMD planes with hits in the drift chambers only (e.g., $K_{s}^{0} \rightarrow \pi^{-} \pi^{+}$or $\Lambda^{0} \rightarrow p \pi^{-}$which have relatively longer lifetimes). PASS1 also made a preliminary calculation of the beam particle trajectory using the information from the upstream MWPC and SMD systems.

\subsubsection{PASS2}

The information obtained from PASS1 was used as input for the following routines: Čerenkov ID, to calculate the particle type probabilities; calorimetry, to match the 
amount of energy deposited by each shower in SLIC and hadrometer with the charged tracks; vertexing, to find all the possible combinations of tracks forming a vertex.

The Cerenkov particle identification program calculated the probability of a charged particle being an electron, muon, pion, kaon, and proton. The probability calculations were based on the light observed in the phototubes (ADC counts), along with the particle momentum and track information. The overall probability $C P R B_{i, j}$ that particle type $i$ and track $j$ were correlated was defined as:

$$
C P R B_{i, j}=P C 1_{i, j} \times P C 2_{i, j} \times A_{i}
$$

where $P C 1$ and $P C 2$ represent the probabilities in counters $\mathrm{C} 1$ and $\mathrm{C} 2$, and $A_{i}$, the $a$ priori likelihood, represents the fraction of $e, \mu, \pi, K$, and $p$ produced in our collisions [22]. The normalization condition was then applied so that:

$$
\sum_{i=1}^{5} C P R B_{i, j}=1
$$

The calorimetry code first started with the measured pedestals and gains obtained during the calibration runs to convert the counter ouputs into physical energies. The shower reconstruction routine consisted of two major parts. The first part found the energy groupings, called sectors, in the calorimeters. The second part generated a list of candidate particles with energy depositions which matched the sectors. For a detailed description of the calorimetry reconstruction program, refer to [31].

The vertex reconstruction started with forming a vertex of two SMD tracks with $\chi^{2}$ per degree of freedom $(D O F)$ less than 2.0. Then, another track from the SMD track list would be added to the vertex and $\chi^{2} / D O F$ recalculated for the new combination. The $\chi^{2}$ for a vertex was defined as the sum of the $\chi^{2}$ (eq. 3.1) of each track with the constraint that all tracks go through the vertex point. If $\chi^{2} / D O F$ was greater than 2.0 , the program would remove the track which contributed the most to the vertex $\chi^{2} / D O F$. This procedure continued until all tracks in the list were tried and a vertex selected. This was then repeated for the remainder of the tracks, not in the previous vertex, to find other vertices. This process was continued until no more vertices were found. At the end, the vertex with the largest number of tracks was selected as the primary. 
After the events were reconstructed, the information which was necessary for the final analysis was compressed and stored on Data Summary Tapes (DSTs). This reduced the number of tapes holding our total data sample by a factor of two compared to the original raw data tapes.

\subsubsection{Computing}

The entire reconstruction process took approximately 20 seconds per event on a 1 MIPS (Million Instructions Per Second) machine such as a Vax 11/780. This translated roughly into a couple of hundred MIPS-yrs of computing to convert our entire raw data sample into DST format. To achieve this goal, we made use of two different systems. About half of the data was reconstructed on ACP farms which on the average consisted of 130 nodes, each with a computing power of 0.7 MIPS. The rest of the data was processed by four Silicon Graphics 4D/240S computers [32]. Each of these computers had four processors of 17 MIPS each. This system alone was about two and a half times more powerful than our ACP farm. Using both systems, we finished the reconstruction of our entire sample of 370 million events in one year.

\subsection{E769 Monte Carlo}

Due to the less than perfect efficiency and limited acceptance of our detectors, only a small portion of the events produced can be reconstructed. In order to extract the actual physics results from our experiment, we need to know the acceptance of our spectrometer. This requires simulation of the production and decay processes and the detection of the particles in the spectrometer. This simulation program is called Monte Carlo (MC).

The Monte Carlo method is defined as representing the solution of a problem as a parameter of a hypothetical population, and using a random sequence of numbers to construct a sample of the population, from which statistical estimates of the parameter can be obtained [33]. In general, a Monte Carlo technique is any technique which makes use of random numbers to solve a problem. 
Our Monte Carlo program consists of two separate parts: the generator, and the digitizer. The generator itself can be divided into two components. The first part contains the code which generates the charm event and the second part simulates the passage of the particles through the spectrometer and their interactions with the detectors. These parts are described in more details in the following sub-sections.

The digitizer part of the Monte Carlo contains all the detector characteristics such as noise, cross talk, and efficiencies. This program converts the generator output into raw data format in order to allow the use of the same processes performed on the real data.

\subsubsection{Event Generation}

This part of the generator consists of three basic phases: the production of the charm quark pair $(c \bar{c})$, the modelling of the hadron-nucleus interaction, and the fragmentation or hadronization of the produced quarks and the beam fragments. It also simulates the decay of the unstable products.

The initial phase was simulated using PYTHIA 4.8 which employs leading order (LO) QCD calculations to generate charm quarks. The mechanism for charm production at the parton level is either gluon fusion $(g+g \rightarrow c \bar{c})$ or quark-antiquark annihilation $(q+\bar{q} \rightarrow c \bar{c})$. See Chapter 2 for more details.

In the next phase, the underlying event was modelled initially by considering only the interaction between the beam particle and a single nucleon. This totally ignored the fact that the nucleon was part of a nucleus, and it subsequently led to a charged particle multiplicity which was about $15 \%$ lower than that for the real data [26]. To correct this shortcoming, we used Fritiof [34], instead. This program was developed by the Lund Monte Carlo Group, designed specifically to model hadronnucleus collisions, which allowed for multiple soft interactions inside the nucleus.

In the final phase, the fragmentation process was implemented by the use of JETSET 6.3, a string fragmentation model developed by the Lund Group [35]. This program also simulates the decays of the unstable particles, using a phase-space decay distribution. The output of these routines was a list, called the Lund List, which contained the 4-momenta of all the particles in the fragmentation-decay chain of the 
initial quarks. An interaction point $(x, y, z)$ was then chosen based on the beam and target characteristics, and the decay times (taken from the PDG [21]) were thrown for the unstable particles [26]. The trajectories were determined by this stage and the particles were then passed through the detector simulation.

\subsubsection{Detector Simulation}

This section of the generator simulates the passage of a particle through the spectrometer and the ensuing interactions such as Coulomb multiple scattering, and secondary interactions with the detector material. For each detector the physical size, the number of interaction lengths, the number of radiation lengths, etc. were programmed into the MC.

The code tracks the path of the charged particles through the magnets, calculates the positions of these particles in each plane of the SMD, MWPC, and DC systems, simulates the emission of the Cerenkov light in $\mathrm{C} 1$ and $\mathrm{C} 2$, and the energy deposited in the calorimeters. The electromagntic shower particles' $\left(\gamma, e^{\mp}\right)$ interactions with the SLIC were simulated using a trimmed-down version of the EGS Monte Carlo. The showering of hadrons in the SLIC and the hadrometer was modelled using a simple average parameterization of the shower depth and width [26].

The output of this part of the generator is known as the "truth table." It contains such information as the positions of the charged particles at each silicon, drift chamber, and proportional wire chamber plane, the amount of light in each Čerenkov phototube, the energy deposited in each calorimeter module, and the full Lund List.

The truth table was then passed on to the digitizer to be translated into raw data format. The digitizer code modelled the effects of the detector inefficiencies, hot and dead channels, noise and cross-talk. The size and form of all these effects were determined from the data. 


\subsection{Signal Extraction}

Due to the large size of our data $(370 \mathrm{M}$ events), in order to extract the signal for the specific modes we planned to study, we first had to reduce our data sample to a manageable size. We knew that only a fraction (1/1000) of our data events included charm interactions. We also knew that the lifetimes of charm mesons such as $D^{0}$ were of the order of a pico-second $\left(10^{-12}\right.$ sec.) which translated into a decay length of a few millimeters in the lab system. This information was used to select the charm candidates for the analysis of different charm decay modes.

The selection process was performed at two stages. First, we used general selection criteria (cuts) to our original data sample to choose the events with at least two wellseparated vertices (pair strip). Next, we applied more specific cuts to our pair strip sample to select specific decay modes we planned to study (mode sub-strip). The strategy was to use a few simple but efficient cuts to get a large reduction factor.

\subsubsection{Pair Strip}

One of the signatures of a charm event is a minimum of three vertices: one interaction point (primary vertex) and two charm decays (secondary vertices). However, due to the limited acceptance of our spectrometer, we often expect to see only one of the secondaries. Consequently, we decided to select the events with at least two vertices.

The criteria for selecting the vertices were as follows. First, we found the two-track pairs from the set of all tracks with hits in the silicon system which passed through at least the first magnet. Then, we checked if either track was in the primary vertex. (The primary was already found by PASS2 vertexing algorithm to be the vertex with the highest multiplicity of tracks.) If either track was (or both tracks were) already included in the primary, the vertex was refitted with the track(s) excluded. If the newly calculated position of the primary was upstream of the target, the event was discarded. Next, the two track combination was fitted to a vertex and the cuts listed in table 4.1 were applied. $\sigma_{z}$, the error in the $z$-coordinate of the secondary vertex, had to be $<1.8 \mathrm{~mm}$, and chi-square per degree of freedom $\left(\chi^{2} / D O F\right)<5$. Vertex pairs which did not pass these cuts were discarded. 


\begin{tabular}{|c|c|}
\hline Variable & Cut \\
\hline \hline$\sigma_{z}$ (secondary) $(\mathrm{cm})$ & $<0.18$ \\
\hline$\chi^{2} / D O F$ (secondary) & $<5$ \\
\hline$S D Z$ & $>6$ \\
\hline$R A T$ & $<0.06$ \\
\hline$P T 2 D K\left(\mathrm{GeV}^{2} / \mathrm{c}^{2}\right)$ & $>0.1$ \\
\hline
\end{tabular}

Table 4.1: Pair strip cuts.

After the application of the vertex quality criteria, we used additional cuts. The significance of the separation between the primary and the secondary was calculated. Since the reconstructed tracks' trajectories were almost parallel to the $z$-axis, we calculated the separation only in this direction $(\Delta z)$, and ignored $\Delta x$ and $\Delta y$. Also, since the longitudinal error for the secondary vertex position was typically 300-500 $\mu \mathrm{m}$ while the transverse resolution was only $\sim 10 \mu \mathrm{m}$, the error in the $z$ direction dominated the error on the vertex position. Thus, the significance was defined as $S D Z \equiv \Delta z / \sigma_{z}$, where $\sigma_{z}=\sqrt{\sigma_{z p}^{2}+\sigma_{z s}^{2}}$ and $\sigma_{z p}$ is the $z$-error on the primary vertex, etc..

Another requirement imposed on the vertices was that the impact parameters of the secondary tracks with respect to the secondary vertex be less than their impact parameters with respect to the primary. This cut was defined as $R A T \equiv \Pi_{i} b_{i}^{s} / b_{i}^{p}$, where $i$ labels the tracks, and $b^{s}$ and $b^{p}$ are the impact parameters to the secondary and primary vertices. This allowed us to exclude tracks which appeared more likely to have originated from the primary than from the decay vertex.

The last cut was on the sum of the squares of the transverse momenta of the secondary tracks with respect to the decay line. This is shown as $P T 2 D K=\Sigma_{i}\left(p_{t}{ }^{2}\right)_{i}$. This cut was useful in getting rid of the background due to nearly collinear tracks with very different momenta. A typical charm decay has a transverse momentum of about $1 \mathrm{GeV} / \mathrm{c}$ which makes it very unlikely to have two nearly collinear tracks. A schematic representation of $S D Z, R A T$, and $P T 2 D K$ are shown in figure 4.1.

If the track pair passed all the above cuts, the event was written to the output tape. The pair strip cuts were selected to be fully efficient with respect to the final analysis 
cuts, while maximizing the rejection factor. The cuts listed in table 4.1 reduced the data by a factor of 13 ( 28M events passed the pair strip) while maintaining an efficiency of $98 \%$ (determined by Monte Carlo studies) with respect to the final analysis cuts.

\subsubsection{Substrip}

The next step in signal extraction was substripping of the pair strip output data for individual decay modes. For $D^{0} \rightarrow K^{-} \pi^{+} \pi^{-} \pi^{+}$mode as with the pair strip, we started with track quality checks such as the requirement of a chi-square cut for each track in the SMD system which passed through at least the first magnet. We checked only the events with 6 or more tracks ( 4 tracks for the decay vertex and at least 2 for the primary) in the SMD. Then we searched for 4-track combinations where one of the tracks was required to pass a Čerenkov probability cut $(K P R O B \geq 0.13)$, to be identified as a kaon, and where the sum of the charges of the four tracks added up to zero. Next, we applied the vertex quality checks on the combinations which had an effective mass $(E M)$ between 1.665 and $2.065 \mathrm{GeV} / \mathrm{c}^{2}$ (a mass window, 400 $\mathrm{MeV} / \mathrm{c}^{2}$ wide and centered at $1.865 \mathrm{GeV} / \mathrm{c}^{2}$ ). The cuts applied to the vertices were similar to those used in the pair strip with the addition of a new cut called DIP (D Impact Parameter). Since the $D^{0}$ candidate supposedly originated from the primary vertex, the sum of the 3-momenta of the four decay tracks should point back to the primary vertex. Using this momentum vector, and the positions of the secondary and the primary vertices, the impact parameter of the parent particle with respect to the primary was calculated. The schematic representation of this cut is also shown in figure 4.1. The summary of the substrip cuts is listed in table 4.2. The application of these cuts provided a rejection factor of 41 , or a sample of $691 \mathrm{~K}$ events, which can be translated into a total rejection factor of $530(\sim 13 \times 41)$ with respect to the original DST sample. 

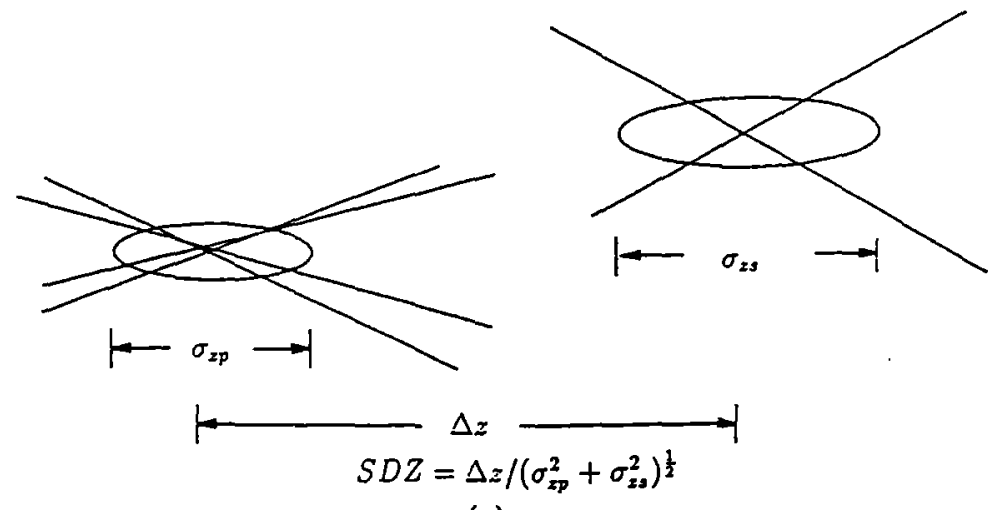

(a)

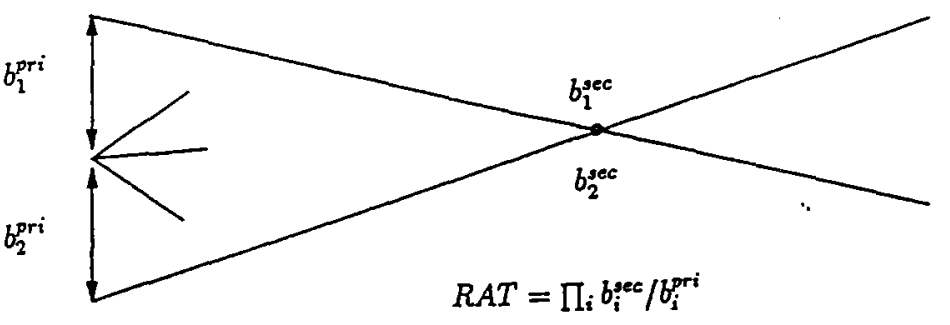

(c)

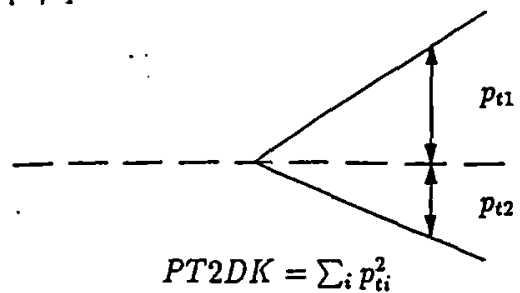

(d)

Figure 4.1: Schematic representation of the principal analysis cuts. 


\begin{tabular}{|c|c|}
\hline Variable & Cut \\
\hline \hline$\sigma_{z}$ (secondary) $(\mathrm{cm})$ & $<0.18$ \\
\hline$K P R O B$ & $>0.13$ \\
\hline$E M$ window $\left(\mathrm{GeV} / \mathrm{c}^{2}\right)$ & 0.4 \\
\hline$\chi^{2} / D O F($ secondary $)$ & $<5$ \\
\hline$S D Z$ & $>6$ \\
\hline$R A T$ & $<0.04$ \\
\hline$D I P(\mathrm{~cm})$ & $<0.02$ \\
\hline
\end{tabular}

Table 4.2: Substrip cuts.

\subsection{3 $D^{0}$ and $D^{*+}$ Signals}

The $D^{0}$ signal was extracted from the substrip sample using similar but tighter cuts described in the previous section. The only new cut added to the list of strip cuts is the isolation cut (ISO) which was introduced to reduce the background due to an event with an extra low momentum track which could pass our other cuts. The isolation cut requires that all tracks in the event which are not from the decay vertex to be a certain distance away from the vertex. This cut is shown in figure 4.1 along with the other cuts, and all the final analysis cuts are listed in table 4.3.

The values of the cuts were set by maximizing the significance $(S / \sqrt{B})$, where $S$ is the signal size from a sample of Monte Carlo events, and $B$ is the number of background events from a real data sample. We used MC events to have a large independent sample, and to minimize a bias by the fluctuations in the statistics of our data signal.

To select the $D^{*+}$ events for the decay mode, $D^{*+} \rightarrow D^{0}\left(K^{-} \pi^{+} \pi^{-} \pi^{+}\right) \pi^{+}$, from our $D^{0}$ substrip sample, we tried combinations of $D^{0}$ candidates with other SMD tracks as the bachelor pions. First, we made sure the bachelor pion track had a charge opposite to that of the $D^{0}$ candidate's kaon track. Then if this combination passed our mass difference cut $\left(\Delta=E M\left(D^{0} \pi^{+}\right)-E M\left(D^{0}\right)\right)$, we would flag it as our $D^{*}$ candidate. If there were more than one $D^{*}$ candidate per $D^{0}$ event, we picked the 


\begin{tabular}{|c|c|c|}
\hline Variable & $D^{0}$ Cut & $D^{*}$ Cut \\
\hline \hline$\sigma_{z}$ (secondary) $(\mathrm{cm})$ & $<0.18$ & $<0.18$ \\
\hline$K P R O B$ & $>0.13$ & $>0.13$ \\
\hline$E M$ window $\left(\mathrm{GeV} / \mathrm{c}^{2}\right)$ & 0.3 & 0.3 \\
\hline$\Delta\left(\mathrm{GeV} / \mathrm{c}^{2}\right)$ & - & $>0.14294$ and $<0.14794$ \\
\hline$\chi^{2} / D O F($ secondary $)$ & $<3$ & $<5$ \\
\hline$\chi^{2} / D O F($ primary $)$ & $<2$ & $<4$ \\
\hline$S D Z$ & $>8$ & $>7$ \\
\hline$D I P(\mathrm{~cm})$ & $<0.006$ & $<0.008$ \\
\hline$R A T$ & $<0.008$ & $<0.01$ \\
\hline$I S O(\mathrm{~cm})$ & $>0.005$ & - \\
\hline
\end{tabular}

Table 4.3: Final analysis cuts.

one with a $\Delta$ value closest to $145.44 \mathrm{MeV}^{1}$. The final set of cuts for the $D^{*}$ events are listed in table 4.3 next to our $D^{0}$ cuts. We can see that the cuts set for $D^{0}$ 's from $D^{*}$ decays are relatively looser since much of the background is eliminated by the selection process for $D^{0} \pi$ combination, described above.

The application of the analysis cuts yields a total of $624 \pm 40 D^{0}$ 's, and $217 \pm 19$ $D^{*}$ s. However, of these two samples we only use those events produced by pions with the beam probability of greater than $90 \%$. Also, to be able to correct the acceptance for our transverse energy $\left(E_{t}\right)$ trigger, we only use the events which passed our $E_{t}$ or $E_{t B}$ triggers. As a result, the final samples we work with consist of $388 \pm 31 D^{0}$ 's, and $127 \pm 14 D^{*}$ 's. The mass distributions for these two data sets are shown in figure 4.2. The signal estimates were obtained by performing maximum likelihood fits to the mass distributions using a gaussian function for both signals, but a linear background for $D^{0}$ and a flat background for $D^{*}$. The gaussian mass peak was fixed at 1.8645 $\mathrm{GeV} / \mathrm{c}^{2}$ for $D^{0}$, and at $2.0101 \mathrm{GeV} / \mathrm{c}^{2}$ for $D^{*}$ [21]. The signal width was set to 0.01 $\mathrm{GeV} / \mathrm{c}^{2}$ consistent with that obtained for the Monte Carlo signals.

\footnotetext{
${ }^{1}$ This was checked with our Monte Carlo not to introduce any bias.
} 

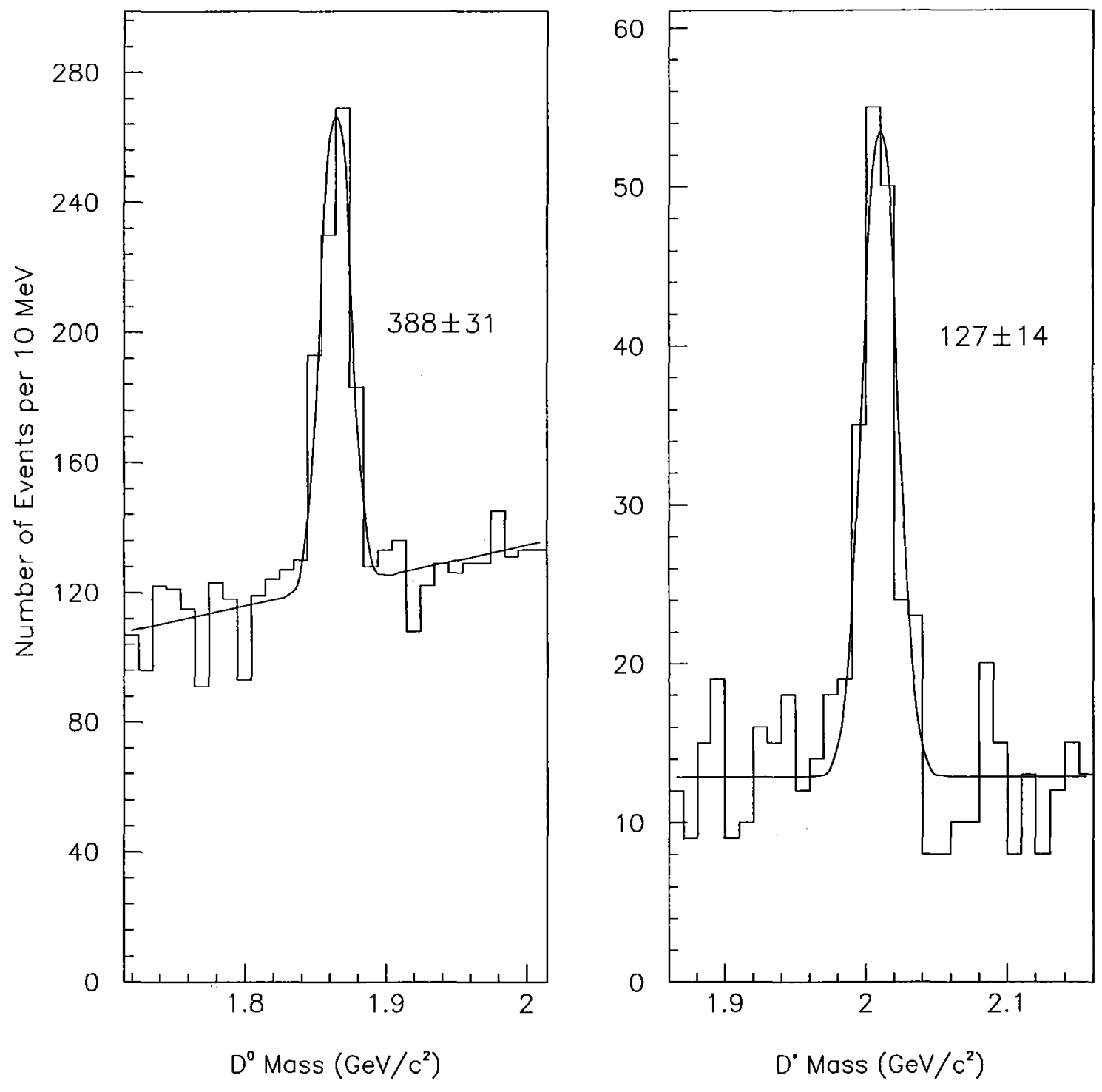

Figure 4.2: $D^{0}$ and $D^{*}$ signals for the pion beam in $E_{t}$ or $E_{t B}$ triggers. 


\section{Chapter 5}

\section{Results}

This chapter presents the results of our analysis of $D^{0} \rightarrow K^{-} \pi^{+} \pi^{-} \pi^{+}$and $D^{*+} \rightarrow$ $D^{0}\left(K^{-} \pi^{+} \pi^{-} \pi^{+}\right) \pi^{+}$decay modes and their charge conjugates. The results mainly deal with the shape parameters of the differential cross section distributions, in terms of the fractional longitudinal momenta $\left(x_{F}\right)$ and the transverse momenta $\left(P_{t}\right)$, of the produced charm mesons.

We start with the mass distributions of the data per bins of $x_{F}$ and $P_{t}^{2}$. Then, we discuss the calculation of the acceptance, and the Čerenkov and trigger efficiency corrections used in our calculation of acceptance. Next, we plot the acceptancecorrected distributions, and fit them to obtain the shape parameters $n$, and $b$. We also discuss the "leading particle" effect, and the asymmetry for the leading versus nonleading particles. Finally, the results of our systematic error analysis are presented.

\subsection{Mass Distributions}

To obtain the differential cross section distributions of $D^{0}$ and $D^{*}$ with respect to the Feynman- $x\left(x_{F}\right)$ and to the transverse momentum $\left(P_{t}\right)$, we start with plotting the mass distributions of each mode per bins of $x_{F}$, or $P_{t}^{2}$. The number of signal events (background subtracted) per bin is determined from a maximum likelihood fit described in section 4.3.3. We keep the width of the signal fixed for all bins (10 $\mathrm{MeV} / \mathrm{c}^{2}$ ). The mass plots for both $x_{F}$ and $P_{t}^{2}$ bins of $D^{0}$ are shown in figures 5.1 and 


\begin{tabular}{|c|c|c|}
\hline$x_{F}$ Range & \# of $D^{0}$ Events & \# of $D^{*}$ Events \\
\hline \hline $0.0-0.1$ & $61.2 \pm 14.6$ & $18.9 \pm 6.3$ \\
\hline $0.1-0.2$ & $135.3 \pm 18.9$ & $42.6 \pm 8.8$ \\
\hline $0.2-0.3$ & $116.5 \pm 15.2$ & $32.5 \pm 6.3$ \\
\hline $0.3-0.4$ & $37.5 \pm 8.5$ & $20.1 \pm 5.1$ \\
\hline $0.4-0.5$ & $32.1 \pm 6.5$ & $9.6 \pm 3.2$ \\
\hline $0.5-0.6$ & $3.4 \pm 2.9$ & $3.0 \pm 1.7$ \\
\hline
\end{tabular}

Table 5.1: Number of $D^{0}$ and $D^{*}$ events per $x_{F}$ bin.

\begin{tabular}{|c|c|c|}
\hline $\begin{array}{c}P_{t}^{2} \text { Range } \\
\left(\mathrm{GeV} / \mathrm{c}^{2}\right)^{2}\end{array}$ & \# of $D^{0}$ Events & \# of $D^{*}$ Events \\
\hline \hline $0-1$ & $213.0 \pm 25.9$ & $72.8 \pm 11.4$ \\
\hline $1-2$ & $84.0 \pm 11.5$ & $7.2 \pm 3.9$ \\
\hline $2-3$ & $38.2 \pm 7.5$ & $17.5 \pm 4.7$ \\
\hline $3-4$ & $26.8 \pm 6.1$ & $10.6 \pm 3.6$ \\
\hline $4-5$ & $11.9 \pm 3.8$ & $10.1 \pm 3.3$ \\
\hline $5-6$ & $5.2 \pm 2.7$ & $2.8 \pm 1.6$ \\
\hline
\end{tabular}

Table 5.2: Number of $D^{0}$ and $D^{*}$ events per $P_{t}^{2}$ bin.

5.2 respectively, and the signal size and the associated error are listed for both $D^{0}$ and $D^{*}$ in tables 5.1-2.

\subsection{Acceptance}

As mentioned in section 4.2, in order to get the correct distributions of our differential cross sections, we need to know the acceptance for each bin of $x_{F}$ and $P_{t}^{2}$. To calculate this, we started with our Monte Carlo (MC) data which after digitization (sec. 4.2.2) went through the same reconstruction process that our raw data events had gone through before (sec. 4.1). After the reconstruction, the events were first pair-stripped (sec. 4.3.1), and then stripped for our specific decay mode. After applying the final 


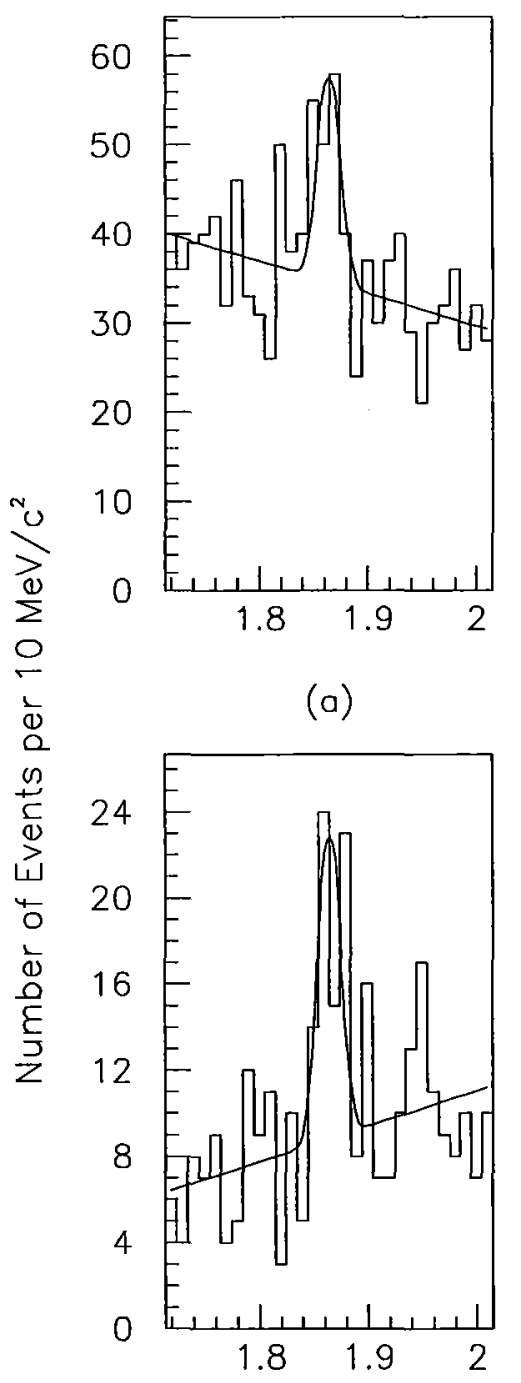

(d)

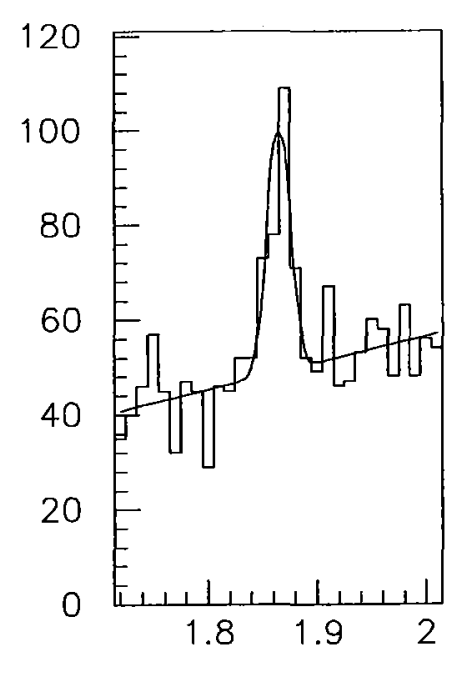

(b)

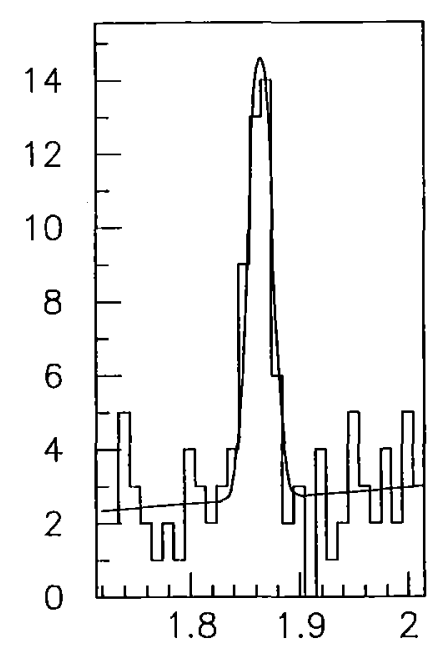

$(\mathrm{e})$
Effective Mass $\left(\mathrm{GeV} / \mathrm{c}^{2}\right)$

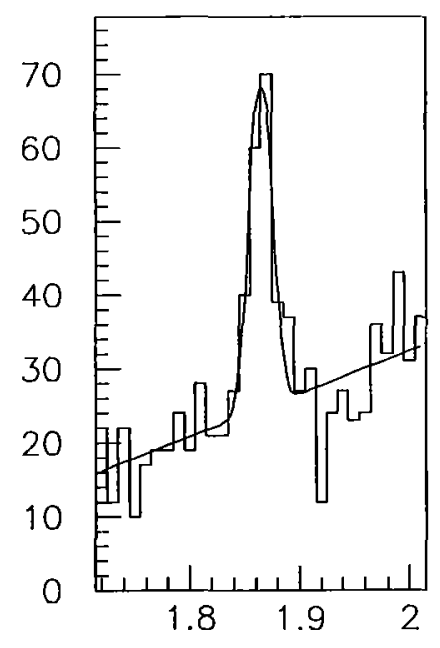

(c)

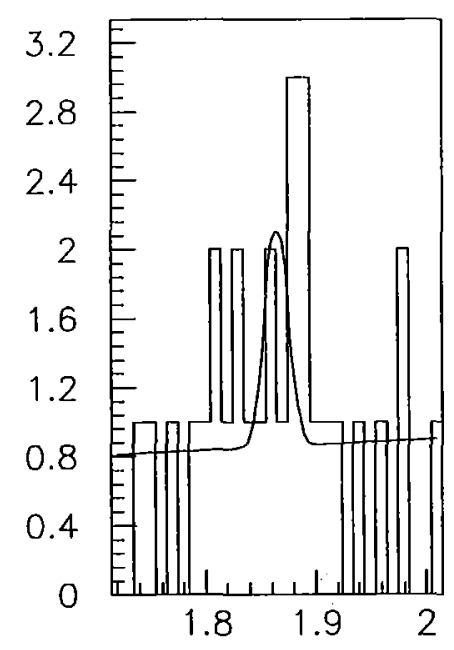

(f)

Figure 5.1: $D^{0}$ mass plots per $x_{F}$ bin. Plots (a) through (f) show the mass distributions in each $x_{F}$ bin of width 0.1 for the range $0.0-0.6$. 


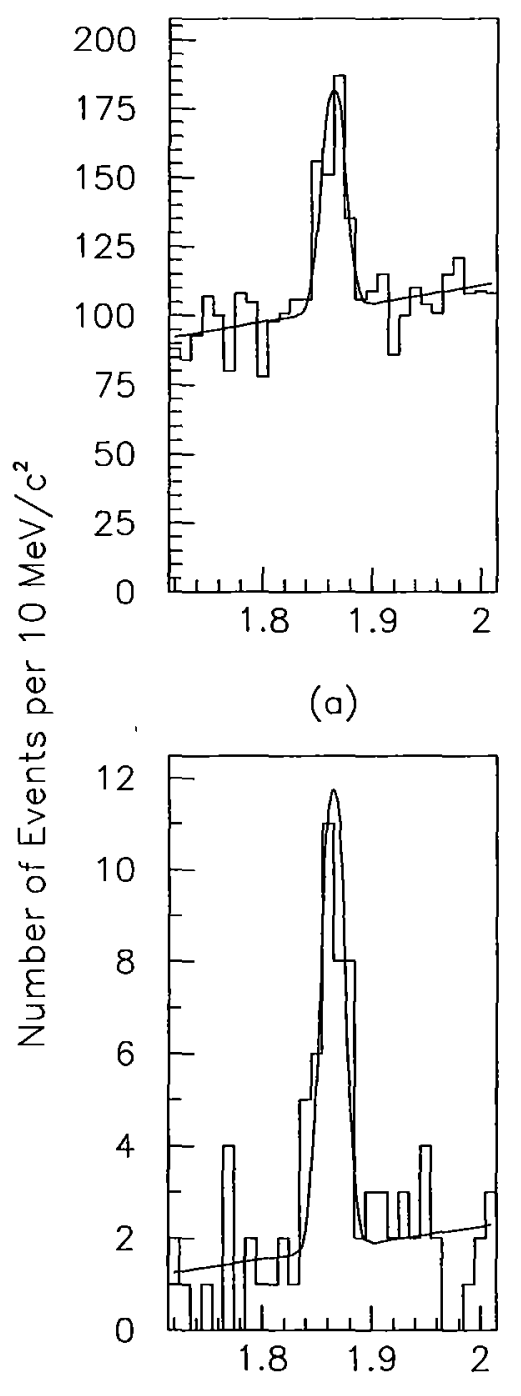

(d)

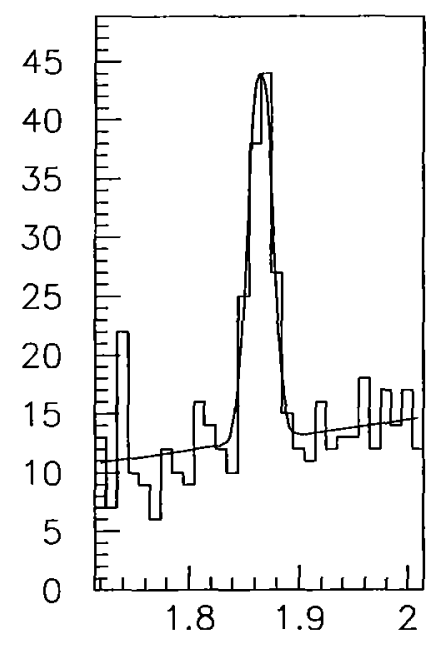

(b)

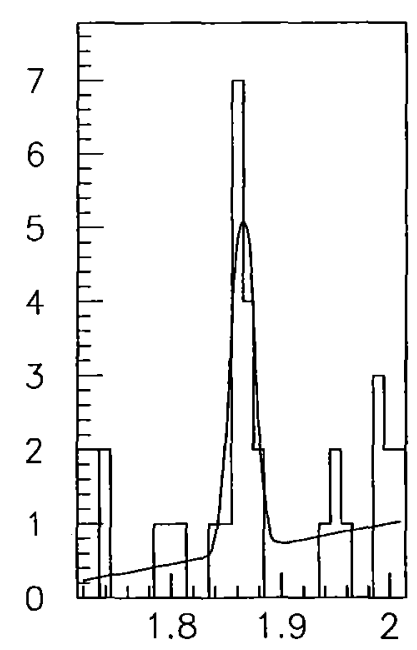

(e)

Effective Mass $\left(\mathrm{GeV} / \mathrm{c}^{2}\right)$

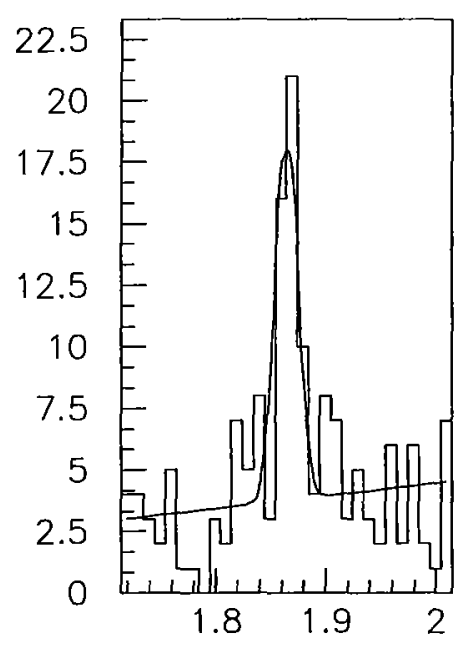

(c)

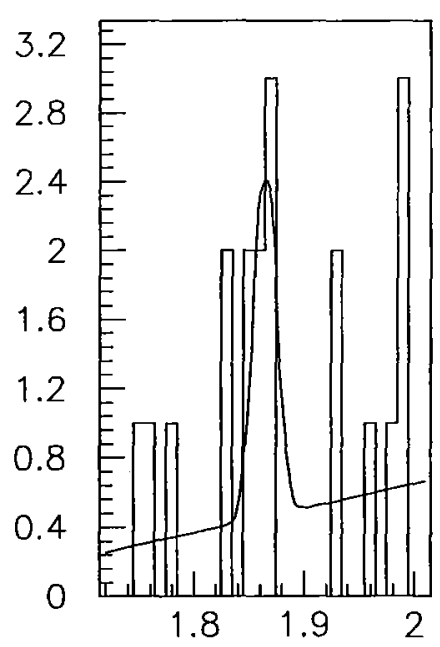

(f)

Figure 5.2: $D^{0}$ mass plots per $P_{t}^{2}$ bin. Plots (a) through (f) show the mass distributions in each $P_{t}^{2}$ bin of unit width for the range $0-6 \mathrm{GeV}^{2} / \mathrm{c}^{2}$. 
cuts, exactly the same as those applied to the real data, we had to weight our MC data to correct for the kaon Cerenkov efficiency and the transverse energy $\left(E_{t}\right)$ trigger efficiency. These corrections are described in more detail in the following subsections.

Having obtained the MC mass distributions per bins of $x_{F}$ and $P_{t}^{2}$, as described in 4.1, we divide the signal size of each bin by the corresponding number of events for that bin in our truth table in order to calculate the acceptance for each bin. The error for acceptance is calculated according to the following equation derived in appendix $\mathrm{A}^{1}$ of reference [27]:

$$
\sigma_{\epsilon}^{2}=\frac{(1-\epsilon) n+(1-2 \epsilon) \sigma_{n}^{2}+\epsilon^{2} \sigma_{N}^{2}}{N^{2}}
$$

where $\epsilon=n / N$ is the acceptance and $\sigma_{n, N}$ is the error of the signal size due to the fit. However, in this case, $N$ represents our truth table events, and its associated error $\left(\sigma_{N}\right)$ is equal to zero.

Due to the deterioration of certain components of our detector system ${ }^{2}$ and the general degraded performance (due to the higher beam intensity) during the positive beam run, our overall efficiency for this period suffered to some extent. This was incorporated into our Monte Carlo, and we calculated two separate acceptances for the negative and the positive runs. These two acceptances, as well as the average acceptance used in our analysis, are shown in figures 5.3 and 5.4 per bin of $x_{F}$ and $P_{t}^{2}$ for the $D^{0}$ mode, while the average acceptances for our $D^{*}$ mode are plotted in figure 5.5.

The average acceptance was calculated by weighting the negative and positive acceptances according to the relative sample size of the real data in the two regions (eq. 5.2). The sample sizes and their relative values for each beam polarity and decay mode are listed in table 5.3.

$$
\frac{1}{A}=\frac{R_{-}}{A_{-}}+\frac{R_{+}}{A_{+}}
$$

$A$ is the acceptance and $R$ is the relative sample size. The small relative size of the positive data has to do with both the lower acceptance ${ }^{3}$ and the smaller size of the pion fraction in the beam composition for the positive runs (sec. 3.1.2).

\footnotetext{
${ }^{1}$ The appendix is titled: "Error Analysis for Correlated Data Samples."

${ }^{2}$ Drift chamber planes D1-2U and $\mathrm{V}$ became inoperable in mid-run.

${ }^{3}$ This is evident in figures $5.3-4$.
} 

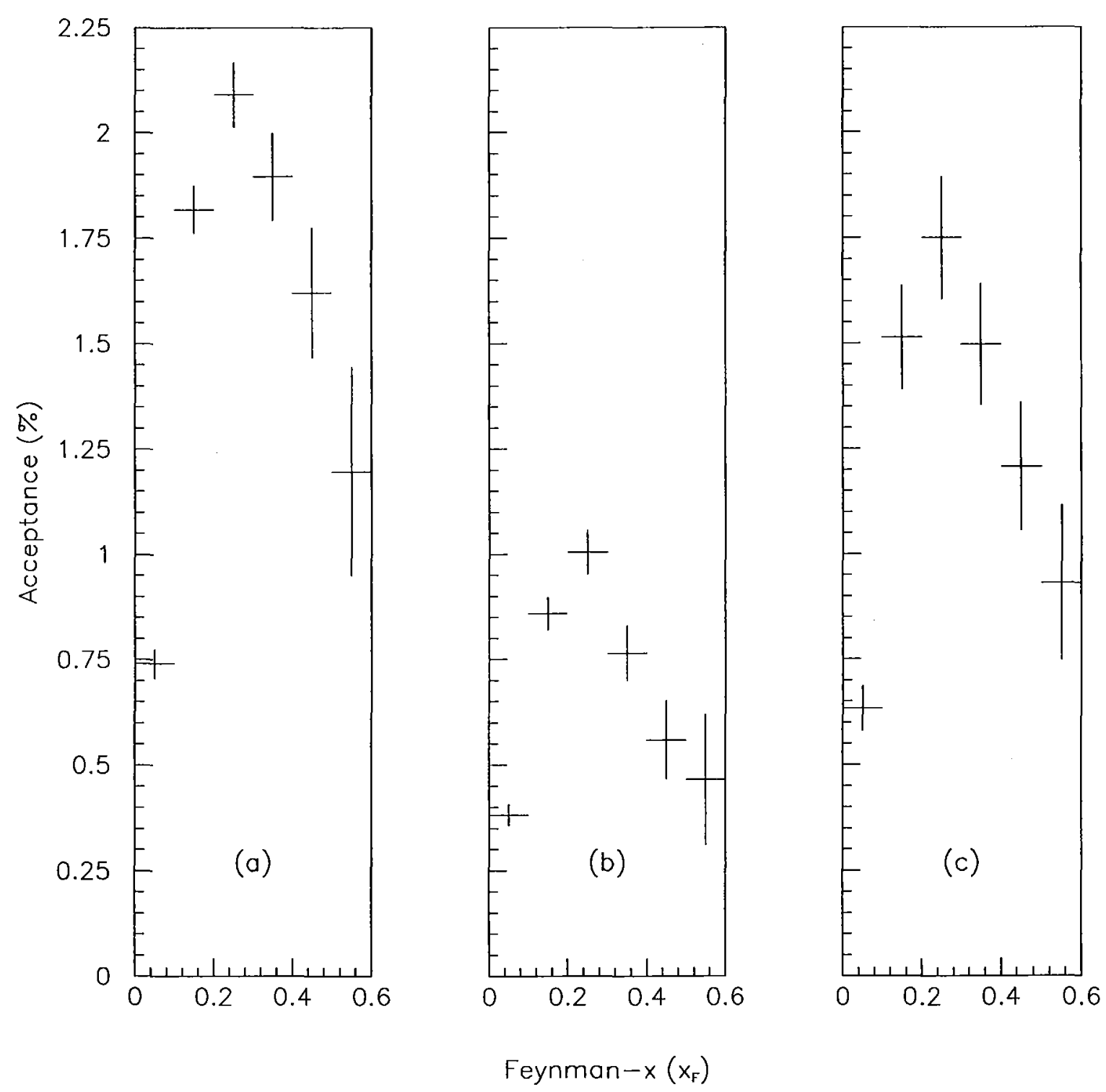

Figure 5.3: $D^{0}$ acceptance plots for (a) the negative runs, (b) the positive runs, and (c) their average per $x_{F}$ bin. 

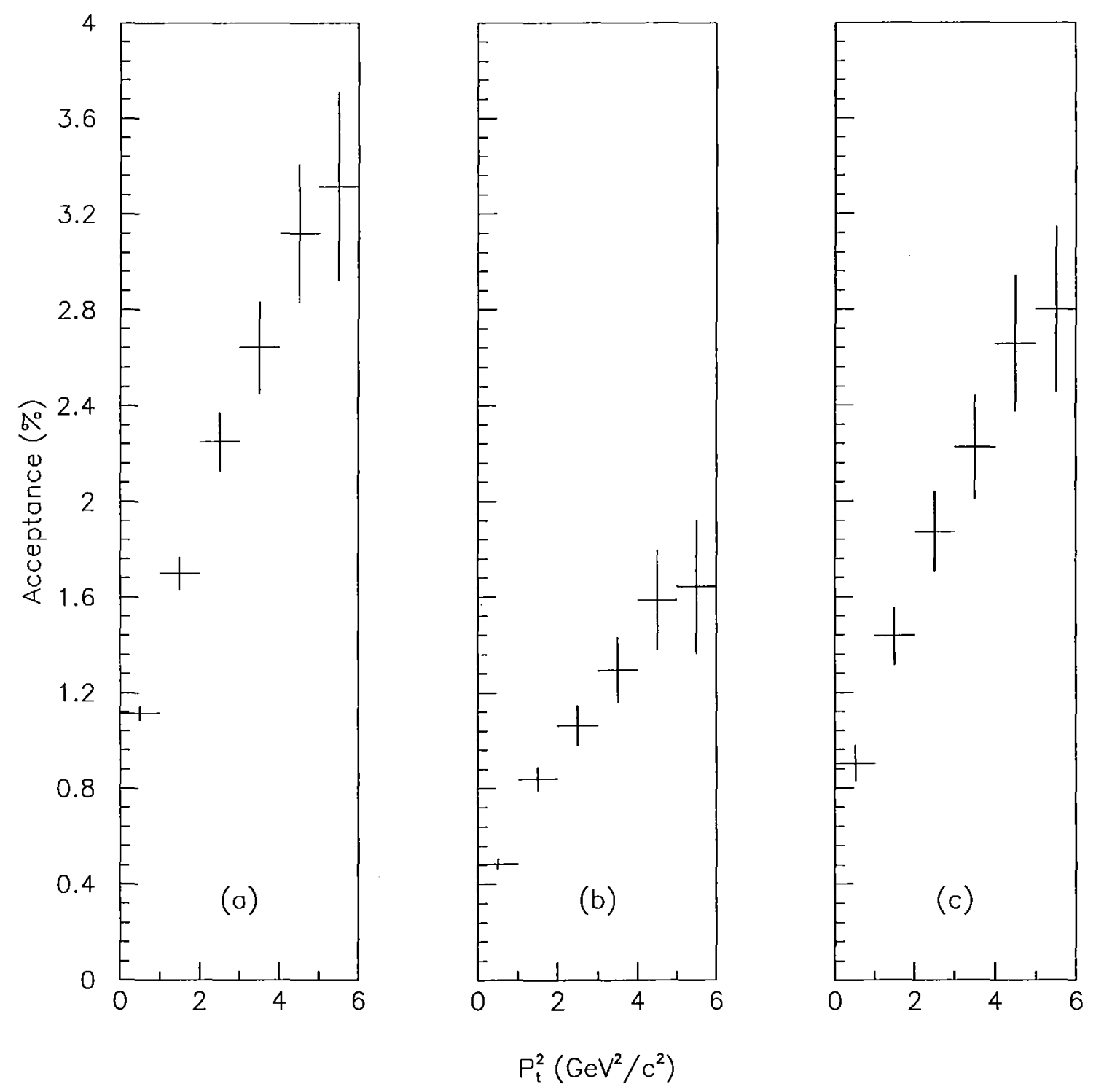

Figure 5.4: $D^{0}$ acceptance plots for (a) the negative runs, (b) the positive runs, and (c) their average per $P_{t}^{2}$ bin. 

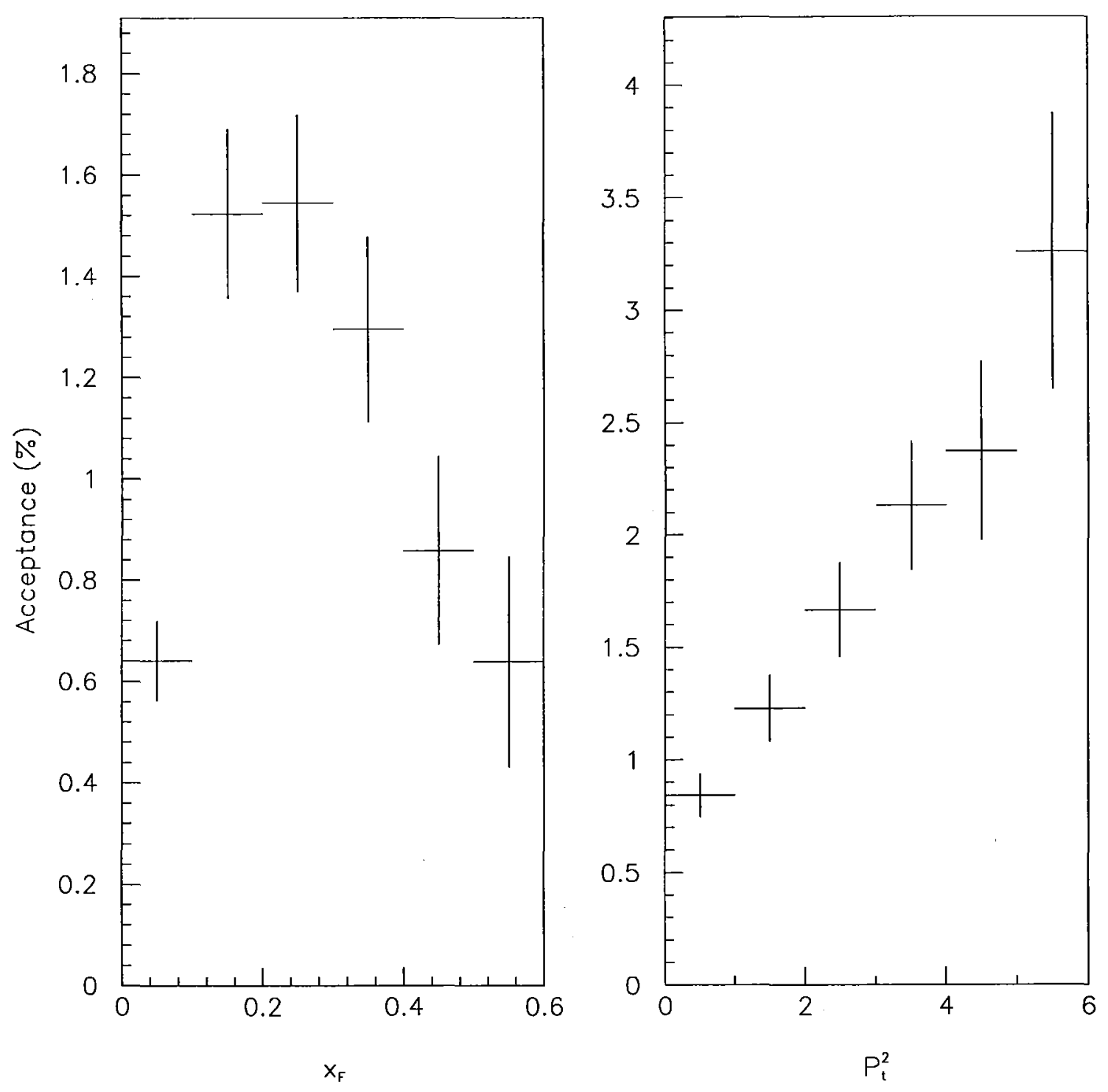

Figure 5.5: $D^{*}$ acceptance plots in bins of $x_{F}$ and $P_{t}^{2}$. 


\begin{tabular}{|c|c|c|c|c|}
\hline Mode & $\begin{array}{c}\text { Signal } \pm \text { Error } \\
(-) \text { beam }\end{array}$ & $\begin{array}{c}\text { Signal } \pm \text { Error } \\
(+) \text { beam }\end{array}$ & $R_{-}$ & $R_{+}$ \\
\hline \hline$D^{0}$ & $320 \pm 27$ & $68 \pm 14$ & $0.82 \pm 0.04$ & $0.18 \pm 0.04$ \\
\hline$D^{*}$ & $104 \pm 13$ & $23 \pm 6$ & $0.82 \pm 0.05$ & $0.18 \pm 0.05$ \\
\hline
\end{tabular}

Table 5.3: Signal size and its relative value in the negative and positive runs for $D^{0}$ and $D^{*}$.

The drop in acceptance for $x_{F}$ near zero is due to the geometry of our spectrometer (suitable for forward interactions), while the lower acceptances for high $x_{F}$ bins are mainly because of our vertex cuts and the DC-hole, and partly due to our trigger. On the other hand, the rise in acceptance for higher $P_{t}^{2}$ values is due to our high transverse energy $\left(E_{t}\right)$ trigger and our analysis cuts, and also the DC-hole is again somewhat responsible for the lower acceptance at low $P_{t}^{2}$ bins.

\subsection{1 Čerenkov Efficiency Correction}

Monte Carlo simulations should correctly mimic the real data. When there is a difference between the data and the $\mathrm{MC}$, there are generally two options available to correct for the discrepancy: to revise the simulation with more accuracy which can be quite involved, or to simply weight the MC by a correction factor determined directly from the data. The first approach is usually taken for more significant differences. Since the Čerenkov kaon efficiency correction was not very large, we opted for the second approach.

The Čerenkov kaon efficiency was studied as a function of the kaon momentum using a large sample of the abundant decay mode $\phi \rightarrow K^{-} K^{+}$. To calculate the efficiency, we made two data samples both for the data and the MC. In one, we required that one of the two kaons have a Čerenkov probability $>0.7$ while no cut was made on the other kaon. In the second sample, the untagged kaon was required to have a Čerenkov probability $>0.13$ (just above the a priori probability). The ratio of the number of $\phi$ 's measured in the second sample to that in the first, for a given range of the untagged kaon momentum, defined our Čerenkov kaon efficiency. 
Figure 5.6 shows these efficiencies for both data and MC in different ranges of the kaon momentum. The momentum ranges were chosen both to cover the interesting thresholds for the Cerenkov radiation (Table 3.3), and to keep the sample size in each range roughly equal[18]. The weight used to correct our MC according to our data distribution is the ratio of the efficiency for the data sample to that for the MC sample.

\subsubsection{Trigger Efficiency Correction}

The analysis presented here used only the events which passed our $E_{t}$ or $E_{t B}$ triggers. As described in section 3.4, these triggers required the event to pass the interaction trigger criterion and to have a total transverse energy above the two thresholds. Our interaction trigger was based on a scintillation counter which produced a signal proportional to the number of charged tracks passing through it. To simulate the interaction trigger in our $\mathrm{MC}$, we parameterized its efficiency versus the number of tracks detected in our silicon system. For a typical charm event with track multiplicity of 10 , the interaction trigger efficiency was $99 \%$ [26].

The transverse energy trigger was harder to model. The gate widths defining the signal integration time were different for the SLIC and the Hadrometer. At the high beam rates, there was a large probability that two beam particles interact in our spectrometer during the timing gates of the calorimeter; one particle (intime) could trigger the readout system while the other (out-of-time) deposited part of its energy during the remaining gate time. Modelling the out-of-time energy was rather difficult. Instead, we chose a different approach which was to parameterize the $E_{t}$ trigger efficiency in terms of a variable named $P_{t 715} . \quad P_{t 715}$ is the sum of the transverse momenta of the charged tracks which pass through both magnets. It has the advantage of being insensitive to the out-of-time energy. For a more detailed description of this method, refer to Appendix A. Figure 5.7 shows the trigger efficiencies versus $P_{t 715}$ for the events in either of the two transverse energy ( $E_{t}$ or $\left.E_{t B}\right)$ triggers.

Our trigger efficiencies were measured relative to the interaction events. In applying them to our MC data, we selected only the MC events which passed our 


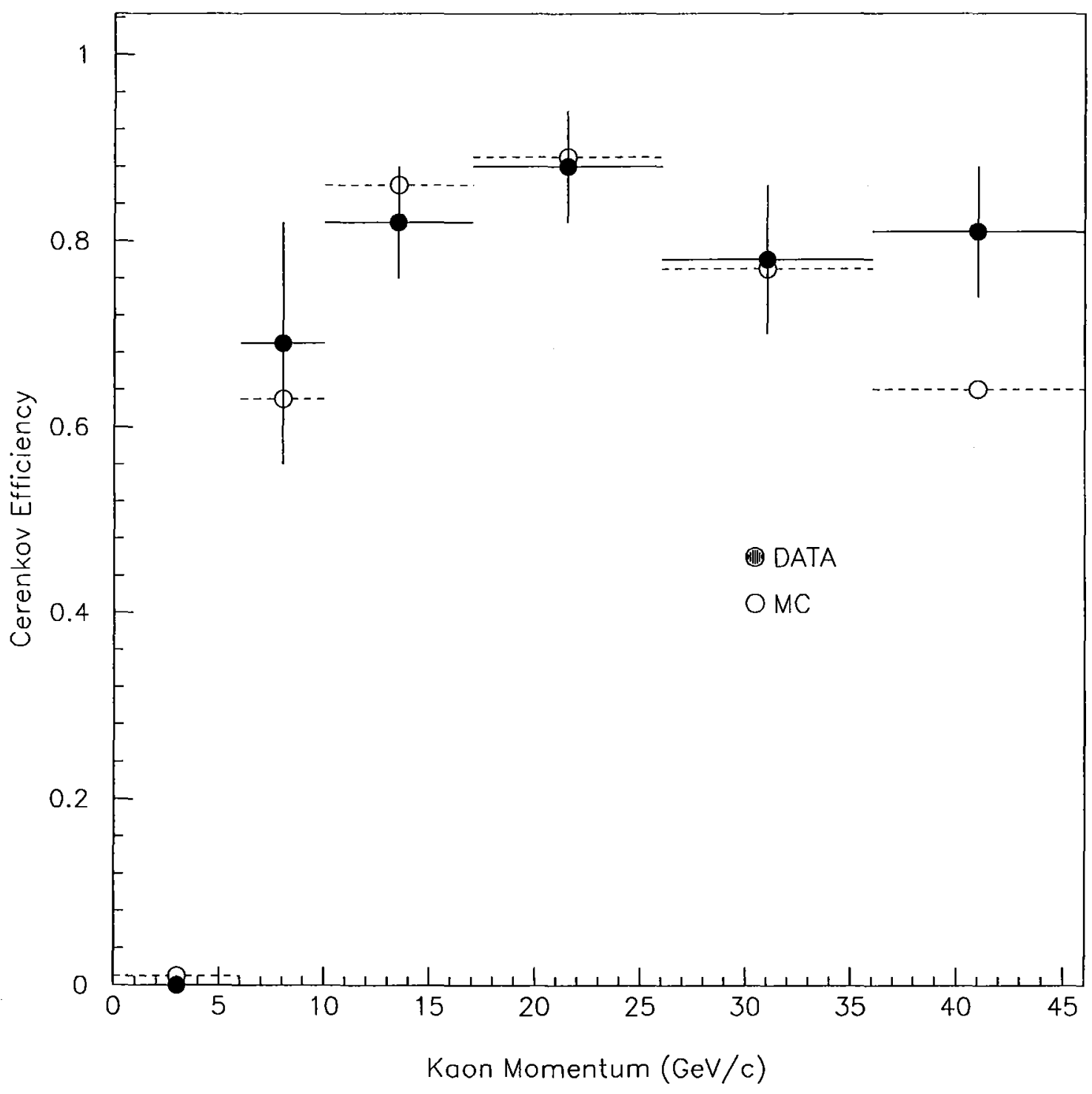

Figure 5.6: Čerenkov kaon efficiency versus kaon momentum for data and MC. 


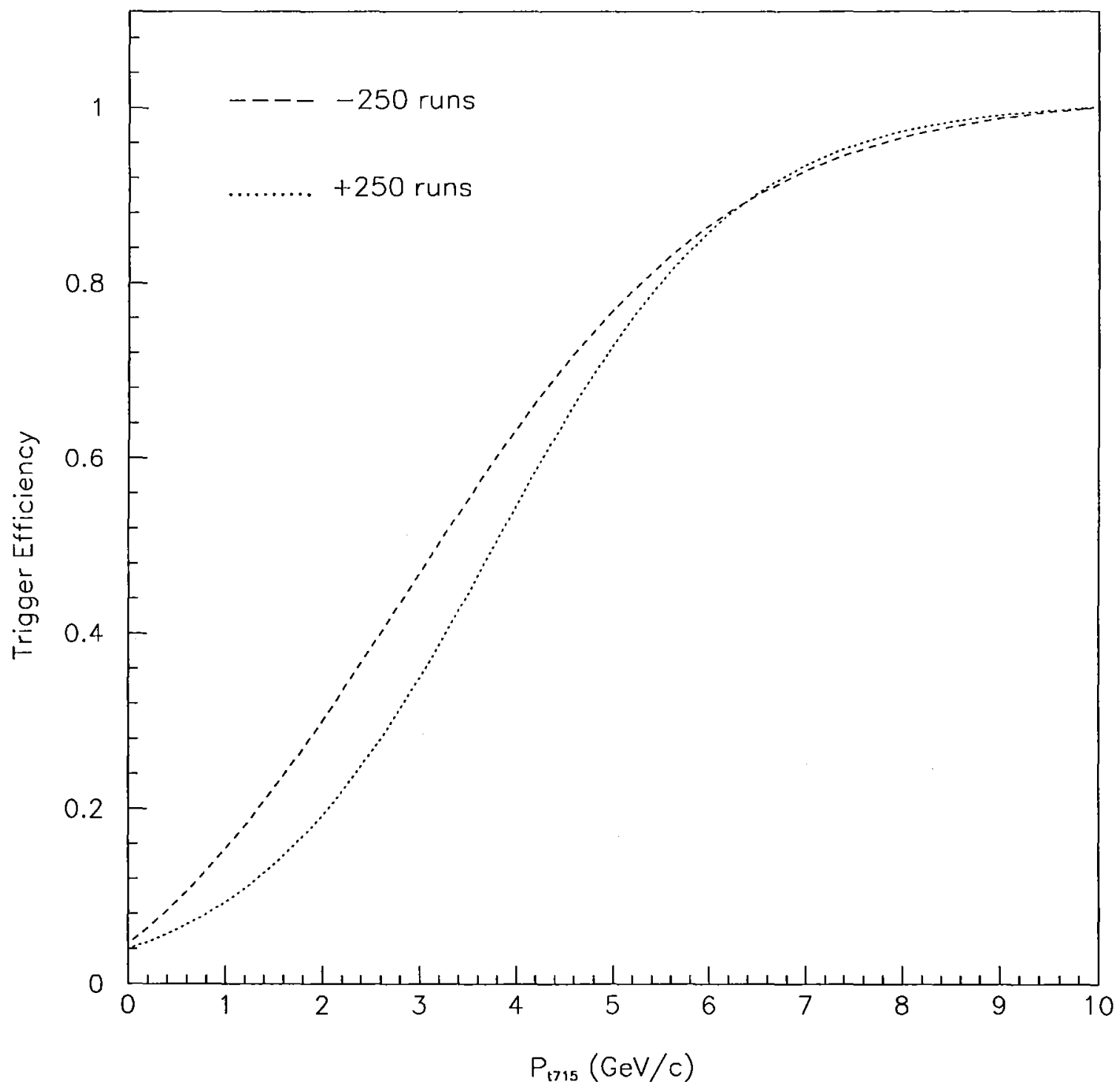

Figure 5.7: $E_{t}$.OR. $E_{t B}$ trigger efficiencies for negative and positive beams. 


\begin{tabular}{|c||c|c|c|}
\hline Shape Parameter & $D^{\mathbf{0}}$ & $D^{\mathbf{0}}$ (Daughter) & $D^{*}$ \\
\hline \hline$n$ & $4.21 \pm 0.54$ & $2.89 \pm 0.86$ & $2.49 \pm 0.70$ \\
\hline$b$ & $1.00 \pm 0.09$ & $0.86 \pm 0.15$ & $0.62 \pm 0.10$ \\
\hline
\end{tabular}

Table 5.4: The shape parameters of the differential cross section distributions for $D^{0}$, $D^{0}$ (daughter), and $D^{*}$.

interaction trigger, and then weighted each event by the amount corresponding to its $P_{t 715}$ value.

\section{3 $\quad x_{F}$ and $P_{t}^{2}$ Distributions}

To obtain the shapes of the differential cross section distributions in terms of $x_{F}$ and $P_{t}^{2}$ variables, we need to correct the results obtained in section 5.1 (tables 5.1-2) for the acceptance calculated in section 5.2. The acceptance-corrected shapes are, therefore, obtained by plotting the ratio of the number of events measured in each bin of $x_{F}$ or $P_{t}^{2}$, divided by the acceptance for that bin. These plots are shown for both $D^{0}$ and $D^{*+}$ in $x_{F}$ and $P_{t}^{2}$ bins in figures 5.8 and 5.9. The shape of each distribution is parameterized by fitting it with one of the functions described below for each independent variable. The fits are applied using the least-squares method.

$$
\begin{gathered}
\frac{d \sigma}{d x_{F}} \propto\left(1-\left|x_{F}\right|\right)^{n} \\
\frac{d \sigma}{d P_{t}^{2}} \propto e^{-b P_{t}^{2}}
\end{gathered}
$$

Table 5.4 lists the values of $n$ and $b$ parameters from the above fits not only for $D^{0}$ and $D^{*+}$ events, but for the $D^{0}$ decays from $D^{*+}$ 's separately. As it is evident from the figures, the fit ranges are $0.1-0.6$ for $x_{F}$, and $0.0-6.0 \mathrm{GeV}^{2} / \mathrm{c}^{2}$ for $P_{t}^{2}$. We do not include the first bin of $x_{F}(0.0-0.1)$ in our fit since despite what equation 5.3 suggests, the distribution is not symmetric about $x_{F}=0$. This is due to the fact that pion-proton interactions, unlike proton-proton, do not have symmetric $x_{F}$ distributions about zero because pions have different structure functions than protons do. 


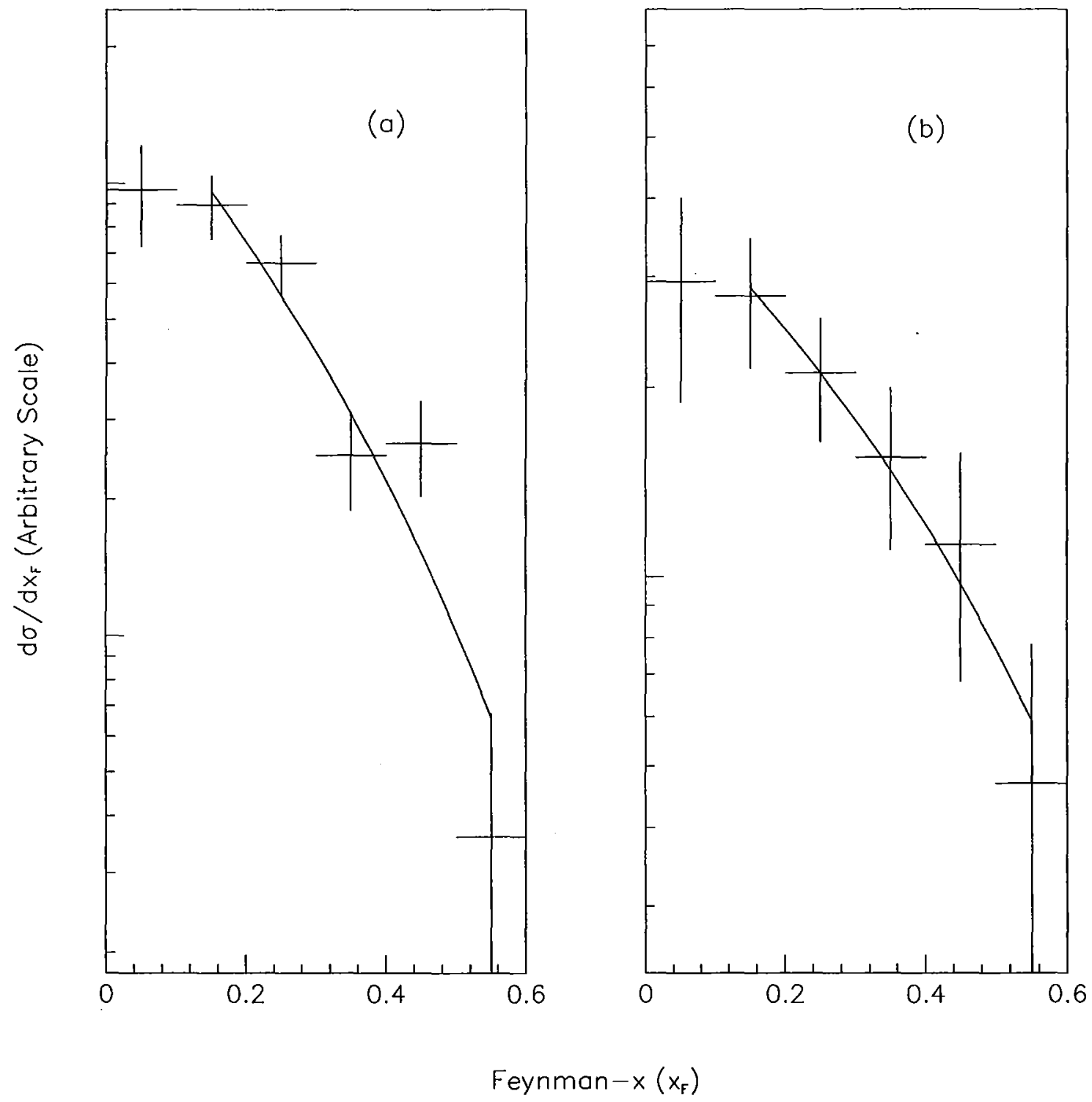

Figure 5.8: Differential cross section distributions for (a) $D^{0}$ and (b) $D^{*}$ in bins of $x_{F}$. 


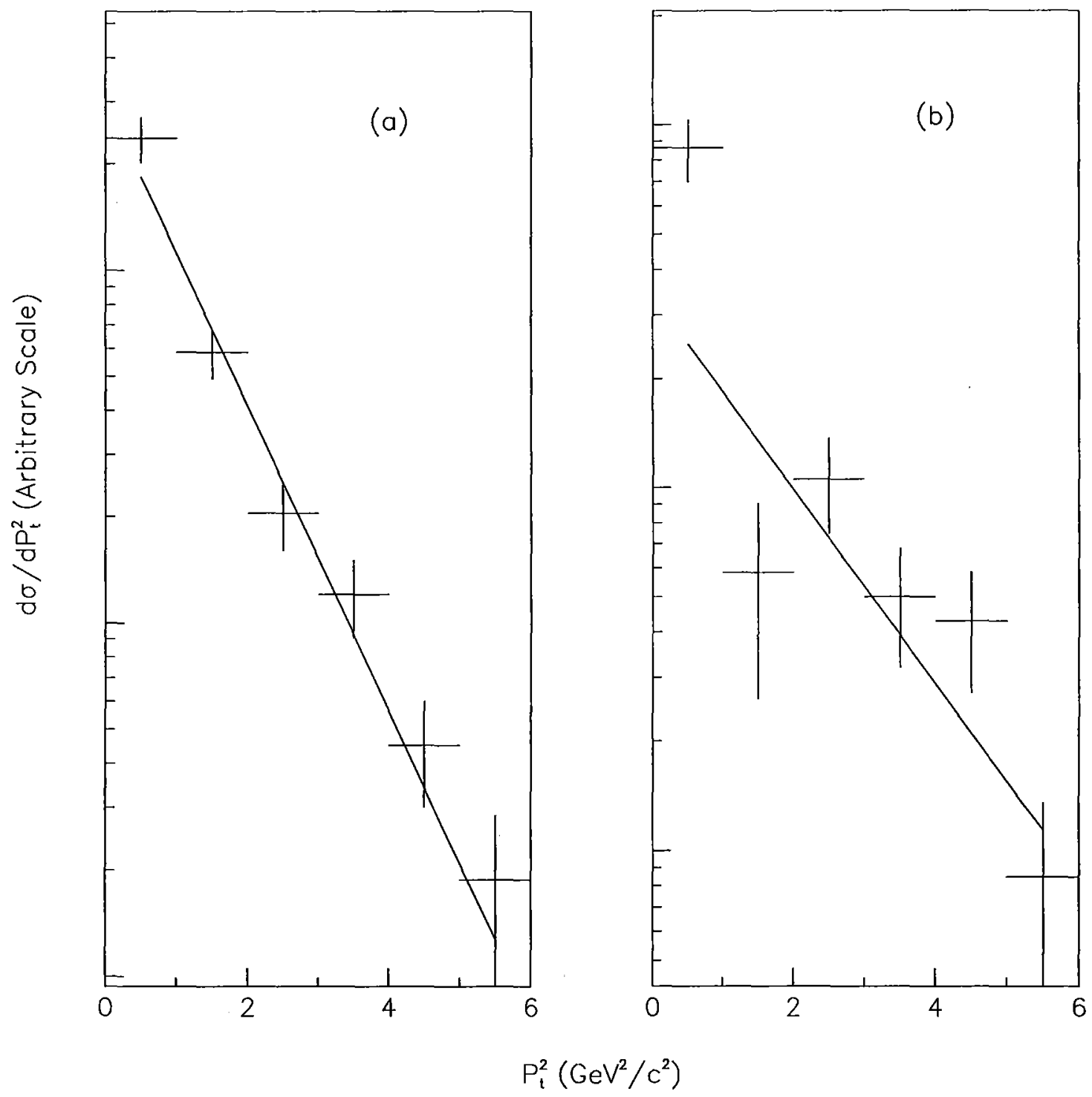

Figure 5.9: Differential cross section distributions for (a) $D^{0}$ and (b) $D^{*}$ in bins of $P_{t}^{2}$. 


\begin{tabular}{|c|c|c|}
\hline Shape Parameter & $D^{*}$ & $D^{0}$ (Daughter) \\
\hline \hline$n_{L}$ & $2.20 \pm 0.79$ & $1.75 \pm 0.94$ \\
$n_{N L}$ & $3.95 \pm 1.10$ & $5.46 \pm 1.32$ \\
\hline$b_{L}$ & $0.45 \pm 0.13$ & $0.67 \pm 0.17$ \\
$b_{N L}$ & $0.75 \pm 0.26$ & $1.00 \pm 0.21$ \\
\hline
\end{tabular}

Table 5.5: The shape parameters of the differential cross section distributions for the leading and the non-leading $D^{*}$ particles and for the corresponding $D^{0}$ (daughter).

\subsubsection{Leading Particle Effect}

In the hadroproducton of charmed hadrons, the charm quark combines with a sea quark or a valence quark from one of the two incident hadrons. When the final state charmed hadron shares a valence quark flavor with the incident hadron beam, it is called a "leading particle". In our case, $D^{*+}$ 's $(c \bar{d})$ produced from $\pi^{+}(u \bar{d})$ beam can be called leading particles since both the charmed particle and the beam particle have an anti-down valence quark. Unlike $D^{*+}$ 's, $D^{0}$ 's are not always directly produced, and as such, their leading/non-leading character is ambiguous. Therefore, to investigate the leading particle effect, we only use our $D^{*}$ data.

We begin with dividing our $D^{*}$ sample into leading and non-leading particles. From the description given in the previous paragraph, it is clear our leading sample contains all $D^{*+}$ 's produced by $\pi^{+}$beam, and all $D^{*-}$ 's from $\pi^{-}$beam. Following the same procedure described for the total sample of our $D^{*}$ 's, we obtain the fit parameters for both the leading and the non-leading sub-samples. The results are shown in table 5.5.

\subsubsection{Asymmetry}

To further investigate the question of leading production, we measure the asymmtery for the leading versus the non-leading $D^{*}$ 's, defined below:

$$
A\left(D_{L}^{*}, D_{N L}^{*}\right)=\frac{\sigma\left(D_{L}^{*}\right)-\sigma\left(D_{N L}^{*}\right)}{\sigma\left(D_{L}^{*}\right)+\sigma\left(D_{N L}^{*}\right)}
$$




\begin{tabular}{|c|c|c||c|c|}
\hline \multirow{2}{*}{ Sources of Error } & \multicolumn{2}{|c||}{$D^{0}$} & \multicolumn{2}{c|}{$D^{*}$} \\
\cline { 2 - 5 } & $\Delta n / n$ & $\Delta b / b$ & $\Delta n / n$ & $\Delta b / b$ \\
\hline \hline Čerenkov Correction & 0.002 & 0.00 & 0.002 & 0.000 \\
\hline Trigger Correction & 0.013 & 0.04 & 0.066 & 0.040 \\
\hline Analysis Cuts & 0.042 & 0.02 & 0.016 & 0.016 \\
\hline \hline Total Systematic Error & 0.044 & 0.045 & 0.068 & 0.043 \\
\hline
\end{tabular}

Table 5.6: Fractional systematic errors of $n$ and $b$ for $D^{0}$ and $D^{*}$.

where $\sigma(X)$ is the acceptance-corrected number of $X$ mesons with $x_{F}>0$. The asymmetry calculated for our $D^{*}$ sample is $0.20 \pm 0.14$. We also measure the differential asymmetry for each bin of $x_{F}$. The plot of these measurements is shown in figure 5.10 .

\subsubsection{Systematic Errors}

All the errors quoted so far have been statistical. The systematic errors in our measurements of the parameters $n$ and $b$ are estimated by studying the uncertainty in the acceptance corrections for the trigger, particle identification, and analysis cuts. To calculate the uncertainty due to each source, we re-measured the parameter without the correction, and used the difference to estimate the error. We took $50 \%$ of the difference as our estimate of the the uncertainty. Table 5.6 lists the uncertainty due to each source, and the total systematic uncertainties for the production parameters of $D^{0}$, and $D^{*}$ modes. These systematic errors turn out to be much smaller than our statistical errors. 


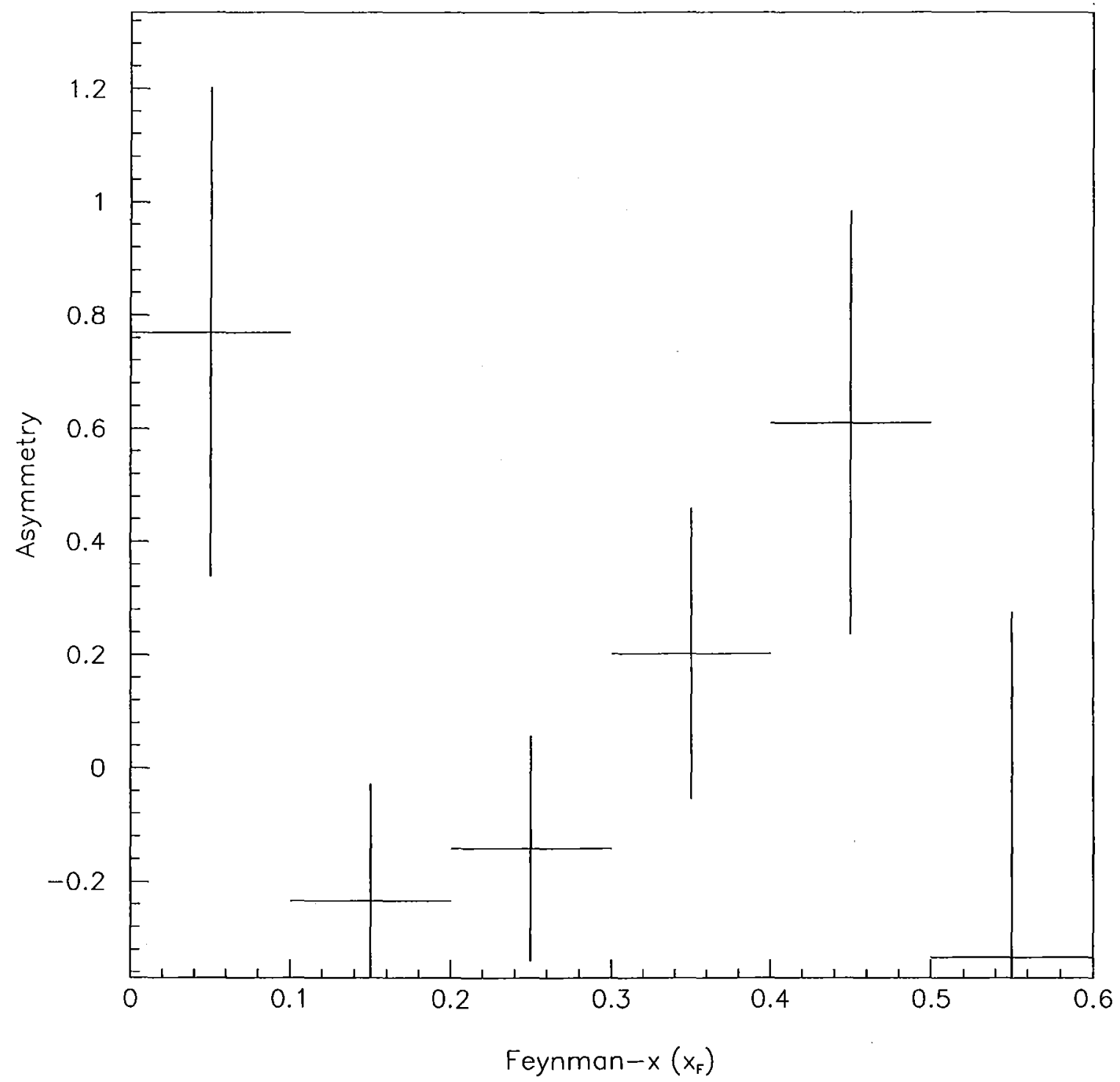

Figure 5.10: Differential asymmetry versus $x_{F}$ for the leading vs. non-leading samples of $D^{*}$ data. 


\section{Chapter 6}

\section{Conclusion}

In this final chapter, we assess the results of our study of the $D^{0} \rightarrow K^{-} \pi^{+} \pi^{-} \pi^{+}$ and $D^{*+} \rightarrow D^{0}\left(K^{-} \pi^{+} \pi^{-} \pi^{+}\right) \pi^{+}$modes by comparing them to results obtained by E769 for other final states of $D^{0}$ and $D^{*+}$ mesons, and to the results of other charm hadroproduction experiments. We then compare the same results to the theoretical calculations in chapter two. We also give an overall summary of our assessments at the end.

\subsection{Comparison to Other Modes}

It is important to compare our results to the similar results pertaining to the production of other final states of $D^{0}$ and $D^{*+}$ since it provides a legitimate method of confirmation. Moreover, we can combine the results of different modes to improve the statistical precision of our data sample. This will put us in a better position to compare our results with those of other experiments, since most experiments often report only the combined results of several final states of a specific charm particle for better statistical accuracy.

In the following two tables, we compare the results of our analysis with other modes of $D^{0}$ and $D^{*+}$ studied by E769. Table 6.1 lists the shape parameters of the differential cross section distributions for $D^{0} \rightarrow K 3 \pi, D^{0} \rightarrow K \pi$ and their combined sample, while table 6.2 shows the values of the same parameters for $D^{*+} \rightarrow$ 


\begin{tabular}{|c||c|c|c|}
\hline Decay Mode & $D^{0}(K 3 \pi)$ & $D^{0}(K \pi)[36]$ & $D^{0}$ (combined) \\
\hline \hline \# Events & $388 \pm 31$ & $607 \pm 41$ & $995 \pm 51$ \\
\hline$x_{F}$ Fit Range & $0.1-0.6$ & $0.1-0.7$ & \\
\hline$n$ & $4.2 \pm 0.5$ & $4.2 \pm 0.5$ & $4.2 \pm 0.4$ \\
\hline$P_{t}^{2}$ Fit Range $\left(\mathrm{GeV}^{2} / \mathrm{c}^{2}\right)$ & $0-6$ & $0-4$ & \\
\hline$b\left(\mathrm{GeV}^{-2} / \mathrm{c}^{-2}\right)$ & $1.00 \pm 0.09$ & $1.08 \pm 0.08$ & $1.04 \pm 0.06$ \\
\hline
\end{tabular}

Table 6.1: Comparison of the production parameters of different $D^{0}$ data samples.

\begin{tabular}{|c||c|c|}
\hline Decay Mode & $D^{*}(K 3 \pi) \pi$ & $D^{*}$ (combined)[37] \\
\hline \hline \# Events & $127 \pm 14$ & $519 \pm 28$ \\
\hline$x_{F}$ Fit Range & $0.1-0.6$ & $0.1-0.6$ \\
\hline$n$ & $2.5 \pm 0.7$ & $3.5 \pm 0.3$ \\
$n_{L}$ & $2.2 \pm 0.8$ & $2.9 \pm 0.4$ \\
$n_{N L}$ & $3.9 \pm 1.1$ & $4.1 \pm 0.5$ \\
\hline$P_{t}^{2}$ Fit Range $\left(\mathrm{GeV}^{2} / \mathrm{c}^{2}\right)$ & $0-6$ & $0-4$ \\
\hline$b\left(\mathrm{GeV}^{-2} / \mathrm{c}^{-2}\right)$ & $0.62 \pm 0.10$ & $0.70 \pm 0.07$ \\
$b_{L}$ & $0.45 \pm 0.13$ & $0.58 \pm 0.09$ \\
$b_{N L}$ & $0.75 \pm 0.26$ & $0.79 \pm 0.09$ \\
\hline
\end{tabular}

Table 6.2: Comparison of the production parameters of different $D^{*}$ data samples.

$D^{0}(K 3 \pi) \pi^{+}$and for the combined sample of three modes: $D^{*+} \rightarrow D^{0}(K \pi) \pi^{+}$, $D^{*+} \rightarrow D^{0}\left(K \pi \pi^{0}\right) \pi^{+}$, and $D^{*+} \rightarrow D^{0}(K 3 \pi) \pi^{+}$. The data samples for all these results include both negative and positive pion beam data for all decay modes, except $D^{0} \rightarrow K \pi$ which includes only the negative pion beam data.

The nearly identical values of $n$ and $b$ for the two different modes of $D^{0}$ are pleasant surprises. Our $D^{*+}$ results do not exactly match, but the parameters in general agree within the large uncertainties associated with $D^{*}(K 3 \pi) \pi$ results. 


\begin{tabular}{|c|c|c|c|}
\hline Expt. & NA32[39] & $\mathrm{E} 653[40]$ & E769 \\
\hline$P_{\text {beam }}(\mathrm{GeV} / \mathrm{c})$ & 230 & 600 & 250 \\
\hline Target & $\mathrm{Cu}$ & Emulsion & $B e, A l, C u, W$ \\
\hline \# Events & 543 & 328 & 388 \\
\hline$x_{F}$ Fit Range & $0.0-0.8$ & $0.0-0.75$ & $0.1-0.6$ \\
\hline$n$ & $3.72 \pm_{0.27}^{0.28}$ & $4.10 \pm 0.31$ & $4.21 \pm 0.54$ \\
\hline$P_{t}^{2}$ Fit Range $\left(\mathrm{GeV}^{2} / \mathrm{c}^{2}\right)$ & $0-10$ & $0-9$ & $0-6$ \\
\hline$b\left(\mathrm{GeV}^{-2} / c^{-2}\right)$ & $0.82 \pm 0.04$ & $0.77 \pm 0.05$ & $1.00 \pm 0.09$ \\
\hline
\end{tabular}

Table 6.3: Comparison of $D^{0}$ production parameters from experiments with incident pion beams.

\subsection{Comparison to Other Experiments}

To compare our results to those of other experiments, we choose the more recent hadroproduction experiments which used pion beams on fixed targets. They are experiments: NA32 [38, 39] at CERN, and E653 [40] at FNAL. Their results along with E769 results are presented for $D^{0}$ and $D^{*+}$ in tables 6.3 and 6.4 respectively. The results quoted for NA32 are for the combined hadronic 2-prong $(K \pi)$ and 4-prong $(K \pi \pi \pi)$ decays of $D^{0}$, and those for $\mathrm{E} 653$ are for the same two modes plus the muonic 2-prong $(K \mu \nu)$. For E769, we show the results of this work only.

Our measurement of the parameter $n$ for $D^{0}$ is in good agreement with the other two measurements, while our value for $b$ is about $2 \sigma$ larger than the others. With respect to the $D^{*+}$ results, our $n$ value seems smaller than the others', but still consistent within the associated errors. The same can be said about our measurements of $b$ parameter, except that our value for $b_{L}$ ( $b$ of the leading particles) is much smaller $(\sim 2 \sigma)$ than the NA32 result.

\subsection{Comparison to Theory}

In this section, we compare the shape parameters of the $x_{F}$ and $P_{t}^{2}$ distributions obtained from our $D^{0}$ and $D^{*+}$ data samples to theoretical predictions. Table 6.5 


\begin{tabular}{|c||ccc|}
\hline Expt. & NA32[38] & NA32[39] & E769 \\
\hline \hline$P_{\text {beam }}(\mathrm{GeV} / \mathrm{c})$ & 200 & 230 & 250 \\
Target & $S i$ & $C u$ & $B e, A l, C u, W$ \\
\hline \hline \# Events & 46 & 147 & 127 \\
\hline$x_{F}$ Fit Range & $0.0-0.7$ & $0.0-0.8$ & $0.1-0.6$ \\
\hline$n$ & $2.8 \pm_{0.9}^{1.1}$ & $3.14 \pm_{0.39}^{0.40}$ & $2.49 \pm 0.70$ \\
$n_{L}$ & $4.7 \pm_{1.9}^{1.9}$ & $2.62 \pm_{0.43}^{0.53}$ & $2.19 \pm 0.79$ \\
$n_{N L}$ & $1.7 \pm_{1.0}^{1.4}$ & $3.83 \pm_{0.62}^{0.66}$ & $3.95 \pm 1.10$ \\
\hline$P_{t}^{2}$ Fit Range $\left(\mathrm{GeV}^{2} / \mathrm{c}^{2}\right)$ & $0-5$ & $0-10$ & $0-6$ \\
\hline$b\left(\mathrm{GeV}^{-2} / \mathrm{c}^{-2}\right)$ & $0.9 \pm_{0.2}^{0.3}$ & $0.79 \pm 0.07$ & $0.62 \pm 0.10$ \\
$b_{L}$ & & $0.71 \pm_{0.08}^{0.09}$ & $0.45 \pm 0.13$ \\
$b_{N L}$ & & $0.90 \pm 0.11$ & $0.75 \pm 0.26$ \\
\hline
\end{tabular}

Table 6.4: Comparison of $D^{*}$ production parameters from experiments with incident pion beams.

\begin{tabular}{|ccccc|}
\hline$D^{*+}$ & $D^{0}$ & $\delta$-Function & Lund & Collins-Spiller \\
\hline \hline $2.5 \pm 0.7$ & $4.2 \pm 0.5$ & 4.0 & 4.1 & 5.0 \\
\hline
\end{tabular}

Table 6.5: Comparison of $n$ parameters with the theoretical calculations.

lists the $n$ values from our data along with the results of our calculations of $n$ for different fragmentation functions given in chapter 2 .

The difference between our $D^{*+}$ and $D^{0}$ results $(2 \sigma)$ is not reflected in our theoretical calculations. One reason is that the theory in its present form does not necessarily differentiate between different charm particles at the hadronization level. Although some fragmentation functions (e.g. Lund's) do include a dependence on the final state hadron mass (eq. 2.7), the result is not so different.

Further comparison of the values in table 6.5 shows a close agreement of our $D^{0}$ result with the $\delta$-function and the Lund fragmentation results. However, there is about a $2 \sigma$ difference between the $D^{0}$ value and the Collins-Spiller prediction and a larger difference between the $D^{*+}$ result and all our theoretical calculations. 
In order to compare our $b$ parameter results with theory, we use the $P_{t}^{2}$ distributions developed by Mangano, Nason, Ridolfi (MNR) in [41]. The fit to their $P_{t}^{2}$ distribution for charm quark production by $230 \mathrm{GeV}$ pion beam in the fit range of $0-6 \mathrm{GeV}^{2} / \mathrm{c}^{2}$ yields $b=1.02$. This agrees very closely with our fit parameter for $D^{0}$ $(b=1.00 \pm 0.09)$. However, the same parameter measured for $D^{*+}(b=0.62 \pm 0.10)$ is $4 \sigma$ smaller than the theoretical prediction for the charm quark.

The apparent agreements or disagreements with the theoretical predictions cannot be considered conclusive due to the large uncertainties associated with the theoretical calculations. The main sources of error are the hadron structure functions, and the fragmentation functions. The structure function parameterizations are heavily dependent on the choice of the QCD mass scale $(\Lambda)$, and the momentum transfer scale $\left(Q^{2}\right)$ (sec. 2.3.1). The fragmentation functions used in our calculations were basically developed from the fits to the charm data produced in $e^{-} e^{+}$annihilations at the center of mass energy of $10.55 \mathrm{GeV}$ [16]. Each fitted parameter in the fragmentation functions had more than $10-20 \%$ error associated with it.

\subsection{Summary}

We have measured the shape parameters of the differential cross section distributions in $x_{F}$ and $P_{t}^{2}$ for $D^{0} \rightarrow K^{-} \pi^{+} \pi^{-} \pi^{+}$and $D^{*+} \rightarrow D^{0}\left(K^{-} \pi^{+} \pi^{-} \pi^{+}\right) \pi^{+}$decay modes, and their charge conjugates. From the comparison with other modes of $D^{0}$ and $D^{*+}$ decays studied by E769, we get reasonable agreements. While our $D^{*+}$ results seem consistently smaller than the combined results of three $D^{*+}$ modes (including ours), our $D^{0}$ parameters are in good agreement with those of $D^{0} \rightarrow K^{-} \pi^{+}$mode.

The comparison with other experiments show that our measurements are generally consistent with the results from the recent charm hadroproduction experiments. Like NA32 [39], we observe a weak leading particle effect $(1.3 \sigma)$, and our total production asymmetry slightly favors the leading particles over the non-leading ones (1.4 $\sigma$ difference). This asymmetry is different from the asymmetry between the $c$ and the $\bar{c}$ productions, predicted by the NLO calculations [13], which is significant mainly at high $x_{F}$. 
In comparison with theory, our $D^{0}$ results seem to be more consistent with the QCD calculations of the charm quark cross section than our $D^{*}$ results are. However, as mentioned in the previous section, the agreements or disagreements with the theory are not necessarily conclusive because of the large uncertainties associated with the theoretical calculations. Therefore, before one can draw any substantive conclusions from these comparisons, all of the uncertainties need to be addressed.

Fortunately, charm hadroproduction is enjoying a surge in new data which will provide the theory with a great opportunity to improve its predictions. Just as E769 with its large data set has been able to contribute to the better measurements of charm hadroproduction parameters, newer experiments such as Fermilab E791 (E769's progeny) with much higher statistics will soon be in a position to not only improve the statistical precision of the previous generation of experiments, but also deal with such important issues as the charge asymmetry predicted by higher order QCD calculations, and the leading particle effect which is still an open question. Therefore, it is safe to say that the immediate future of charm hadroproduction is bright. 


\section{Appendix A}

\section{Trigger Efficiency Functions}

To account for E769 transverse energy $\left(E_{t}\right)$ trigger in the Monte Carlo data, we developed a set of functions to represent the efficiencies for different trigger types during different run periods. In this appendix, we give a general description of how these functions came about.

Early in the course of our study, we realized that the transverse energy measured in our calorimeters was not the best choice of variable for the trigger efficiency parameterization. The fact was that the timing gates defining the signal integration time for the calorimeters (200 ns) were wider than those for the tracking systems (150 ns for DCs and $100 \mathrm{~ns}$ for SMDs). The consequence of this time differential was that at high beam rates, there was a large probability of two beam particles in nearby buckets arriving during the calorimeter timing gates. While the first particle would initiate an interaction and start the readout sequence, the second one could only deposit part of its energy within the remaining time window. This would give the impression of a single particle depositing an energy larger than its true value.

To overcome the problem of out-of-time energy, we defined the variable $P_{t 715}$ as an in-time analog of transverse energy. $P_{t 715}$ is the sum of the transverse momenta of all reconstructed tracks which passed through both of our analysis magnets. To calculate the efficiency, we used the interaction trigger events in our pair-strip data sample. The efficiency as a function of $P_{t 715}$ for each trigger was defined as the fraction of the interaction events which passed the trigger in each $P_{t 715}$ bin. It must be added 


\begin{tabular}{|c||c|c|c|c|}
\hline Run Region & $P_{1}$ & $P_{2}$ & $P_{3}$ & $P_{4}$ \\
\hline \hline 1 & $0.529 \pm 0.0165$ & $0.571 \pm 0.0493$ & $2.127 \pm 0.1213$ & $1.226 \pm 0.1252$ \\
2 & $0.298 \pm 0.0042$ & $0.336 \pm 0.0099$ & $2.849 \pm 0.0698$ & $1.663 \pm 0.0616$ \\
3 & $0.114 \pm 0.0044$ & $0.115 \pm 0.0058$ & $3.767 \pm 0.0856$ & $1.263 \pm 0.0835$ \\
4 & $0.020 \pm 0.0025$ & $0.016 \pm 0.0043$ & $2.957 \pm 0.2701$ & $0.557 \pm 0.3423$ \\
\hline
\end{tabular}

Table A.1: Fit parameters for $E_{t}$.OR. $E_{t B}$ trigger efficiency function.

that since the interaction events were prescaled and the prescaler setting changed during the run, each interaction event was first weighted relative to its corresponding prescaler value, before it was counted. Also, we divide our data sample into four run regions of: $-210 \mathrm{GeV},-250 \mathrm{GeV},+250 \mathrm{GeV}$, and proton beam.

We parameterized our trigger efficiencies, using the following formula which seemed to describe the fit functions best.

$$
\epsilon_{t r i g}=P_{1}-\frac{P_{2}}{1+\exp \left(\frac{P_{t 715}-P_{3}}{P_{4}}\right)}
$$

Figure A.1 shows the efficiency histograms for $E_{t}$.OR. $E_{t B}$ trigger, fitted to the above function, in all four run regions, and table A.1 lists the results of the fits. As it is evident from the figures, the fit functions do not seem to plateau at $100 \%$ for higher $P_{t 715}$ values. This is basically due to such factors as beam prescaler settings and "killer bit" (See sec. 3.4). We normalize these functions to reach $100 \%$ at $P_{t 715}=10$ $\mathrm{GeV} / \mathrm{c}$ before using them for the correction of our MC data (See Fig. 5.7). 


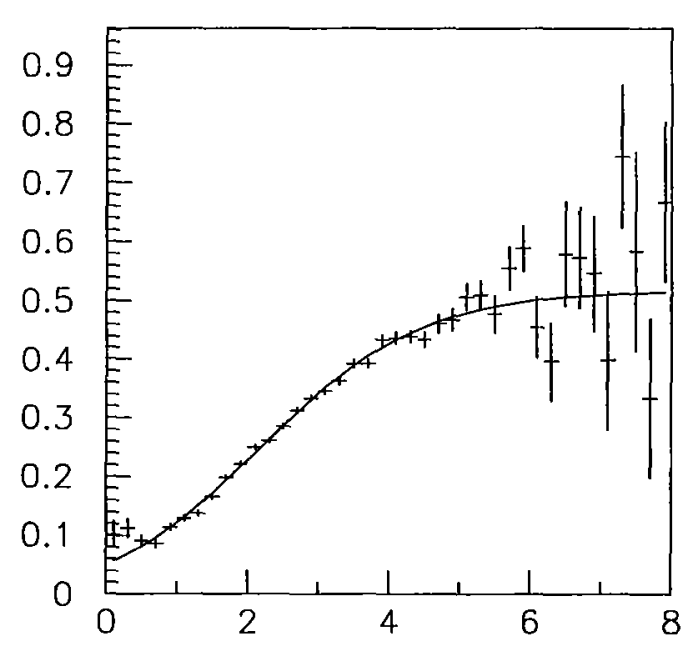

(a)

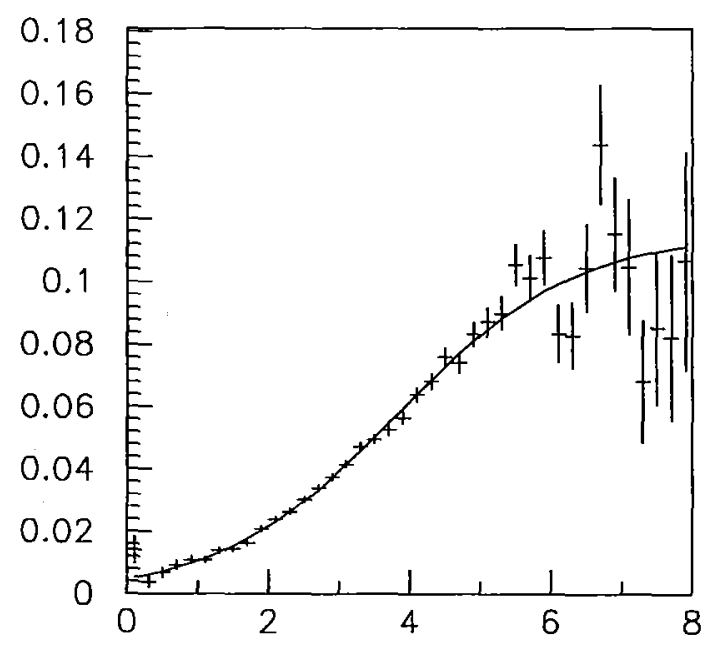

(c)

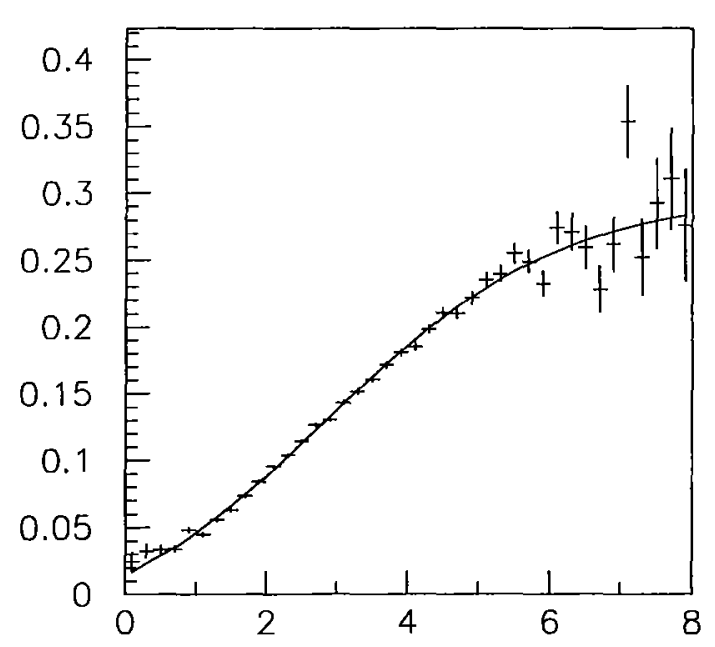

(b)

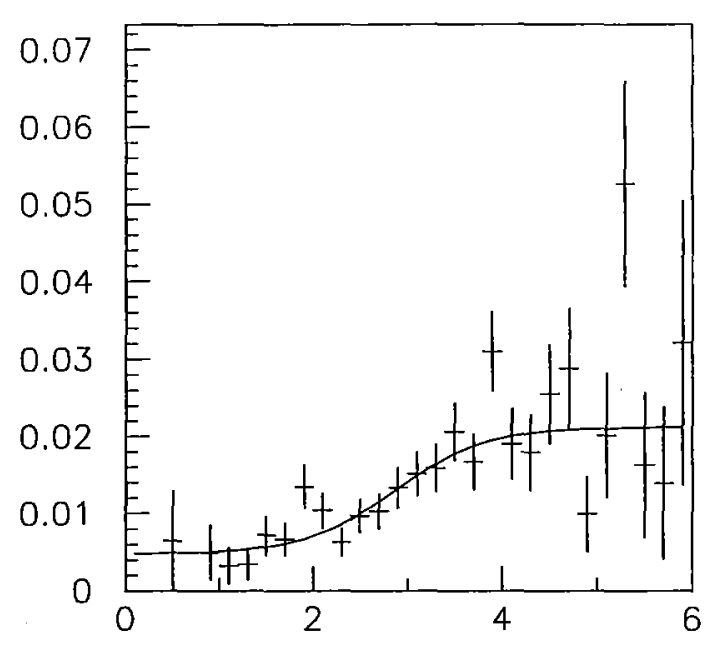

(d)

Figure A.1: Efficiency vs $P_{t 715}$ for $E_{t}$.OR. $E_{t B}$ trigger in (a) $-210 \mathrm{GeV}$, (b) $-250 \mathrm{GeV}$, (c) $+250 \mathrm{GeV}$, (d) proton run regions. 


\section{Bibliography}

[1] S.P.K. Tavernier, Rep. Prog. Phys. 50, 1439 (1987).

[2] J.A. Appel, Annu. Rev. Nucl. Part. Sci. 42, 367 (1992).

[3] M. Gell-Mann, Phys. Lett. 8, 214 (1964).

[4] G. Zweig, CERN Report 8182/TH.401

[5] B.J. Bjorken and S.L. Glashow, Phys. Lett. 11, 255 (1964).

[6] S.L. Glashow, J. Iliopoulos, and L. Maiani, Phys. Rev. D2, 1285 (1970).

[7] J.J. Aubert et al., Phys. Rev. Lett. 33, 1404 (1974).

[8] J.E. Augustin et al., Phys. Rev. Lett. 33, 1406 (1974).

[9] O.W. Greenberg, Phys. Rev. Lett. 13, 598 (1964).

[10] F. Halzen and A.D. Martin, Quarks and Leptons, John Wiley and Sons, New York, (1984).

[11] D.W. Duke and J.F. Owens, Phys. Rev. D30, 49 (1984).

[12] J.F. Owens, Phys. Rev. D30, 943 (1984).

[13] P. Nason, S. Dawson, and R.K. Ellis, Nucl. Phys. B327, 49 (1989).

[14] M. Diemoz et al., Zeitschrift für Physik C39, 21 (1988).

[15] T. Sjöstrand, Int. J. Mod. Phys. A3, 751 (1988). 
[16] D. Bortoletto et al., Phys. Rev. D37, 1719 (1988).

[17] R.C. Fernow, Introduction to Experimental Particle Physics, Cambridge University Press, Cambridge (1986).

[18] R. Jedicke, Flavor Dependence of Hadroproduced Charm-Strange Mesons, Ph.D. Thesis, University of Toronto, 1991.

[19] G. Alves et al., Phys. Rev. Lett. 70, 722 (1993).

[20] D.H. Perkins, Introduction to High Energy Physics, Addison-Wesley, Reading, MA (1982).

[21] Particle Data Group, Phys. Rev. D45, III.5 (1992).

[22] D. Bartlett et al., Nucl. Instr. Meth. A260, 55 (1987).

[23] D. Errede, M. Sheaff, H. Fenker, L. Lueking, P. Mantsch, and R. Jedicke, Nucl. Instr. Meth. A309, 386 (1991).

[24] F. Sauli, Principles of Operation of Multiwire Proportional and Drift Chambers, CERN Report 77-09 (1977).

[25] H. Fenker, A Standard Beam PWC for Fermilab, Fermilab TM-1179 (1983).

[26] C.W. Gay, The Charm Cross Section and Atomic Number Dependence in $\pi^{-} N$ collisions, Ph.D. Thesis, University of Toronto, 1991.

[27] Z. Wu, The Feynman-X Dependence of $D^{ \pm}$Mesons in $\pi^{-}-$Nucleon Interactins, Ph.D. Thesis, Yale University, 1991.

[28] V.K. Bharadwaj et al., Nucl. Instr. Meth. 228, 283 (1985).

[29] J.A. Appel, P.M. Mantsch, M.M. Streetman, and R.M. Robertson, Nucl. Instr. Meth. A243, 361 (1986).

[30] C. Gay, and S. Bracker, The E769 multiprocessor based data acquisition system, IEEE Transactions on Nuclear Science 34, 870 (1987). 
[31] D.J. Summers, Nucl. Instr. Meth. 228, 290 (1985).

[32] C. Stoughton, D.J. Summers, Comp. in Phys. 6, 371 (1992).

[33] T. Ferbel, Experimental Techniques in High Energy Physics, Addison-Wesley, Menlo Park, (1987).

[34] B. Anderson, G. Gustafson, and B. Nilsson-almqvist, A High Energy String Dynamics Model for Hadronic Interactions, University of Lund, LU-TP-87-6, (1987).

[35] B. Anderson, G. Gustafson, G. Ingelman, and T. Sjöstrand, Physics Reports 97, 32 (1983).

[36] G.A. Alves et al., Phys. Rev. Lett. 69, 3147 (1992).

[37] G.A. Alves et al., Fermilab Preprint FERMILAB-Pub-93/081-E (1993).

[38] S. Barlag et al., Z. Phys. C39, 451 (1988).

[39] S. Barlag et al., Z. Phys. C49, 555 (1991).

[40] K. Kodama et al., Phys. Lett. B284, 461 (1992).

[41] M.L. Mangano, P. Nason, and G. Ridolfi, INFN Preprint, IFUP-TH-37 (1992). 